\title{
Finite Time Blowup for Lagrangian Modifications of the Three-Dimensional Euler Equation
}

\author{
Terence Tao ${ }^{1}$
}

Received: 28 June 2016 / Accepted: 14 November 2016 / Published online: 1 December 2016

(C) Springer International Publishing AG 2016

Abstract In the language of differential geometry, the incompressible inviscid Euler equations can be written in vorticity-vector potential form as

$$
\begin{aligned}
\partial_{t} \omega+\mathcal{L}_{u} \omega & =0 \\
u & =\delta \tilde{\eta}^{-1} \Delta^{-1} \omega
\end{aligned}
$$

where $\omega$ is the vorticity 2 -form, $\mathcal{L}_{u}$ denotes the Lie derivative with respect to the velocity field $u, \Delta$ is the Hodge Laplacian, $\delta$ is the codifferential (the negative of the divergence operator), and $\tilde{\eta}^{-1}$ is the canonical map from 2-forms to 2-vector fields induced by the Euclidean metric $\eta$. In this paper we consider a generalisation of these Euler equations in three spatial dimensions, in which the vector potential operator $\tilde{\eta}^{-1} \Delta^{-1}$ is replaced by a more general operator $A$ of order -2 ; this retains the Lagrangian structure of the Euler equations, as well as most of its conservation laws and local existence theory. Despite this, we give three different constructions of such an operator $A$ which admits smooth solutions that blow up in finite time, including an example on $\mathbb{R}^{3}$ which is self-adjoint and positive definite. This indicates a barrier to establishing global regularity for the three-dimensional Euler equations, in that any method for achieving this must use some property of those equations that is not shared by the generalised Euler equations considered here.

Keywords Euler equation · Vorticity formulation · Finite time blowup

Mathematics Subject Classification 35Q30

\footnotetext{
Terence Tao

tao@math.ucla.edu

1 UCLA Department of Mathematics, Los Angeles, CA 90095-1555, USA
} 


\section{Introduction}

\subsection{Formal Theory of the Generalised Euler Equations}

In this paper we will consider finite time blowup for generalised equations of Euler type on Euclidean spaces $\mathbb{R}^{d}$, and more generally ${ }^{1}$ on flat cylinders $\mathbb{R}^{m} \times(\mathbb{R} / \mathbb{Z})^{d-m}$ for $0 \leqslant m \leqslant d$, thus $d$ represents the total number of spatial dimensions, and $m$ the total number of unbounded spatial dimensions. We will restrict attention primarily to the domains $\mathbb{R}^{3}, \mathbb{R}^{2} \times \mathbb{R} / \mathbb{Z}$, and $\mathbb{R}^{2}$. In particular we shall assume that $m \geqslant 2$, in order to avoid some technical issues involving the Biot-Savart law at low frequencies.

Recall that if $\mathcal{M}=\mathbb{R}^{m} \times(\mathbb{R} / \mathbb{Z})^{d-m}$ with $d \geqslant m \geq 2$, the Euler equations for incompressible, inviscid fluids on $\mathcal{M}$ can be written as

$$
\begin{aligned}
\partial_{t} u+(u \cdot \nabla) u & =-\nabla p \\
\nabla \cdot u & =0
\end{aligned}
$$

where $u: \mathbb{R} \times \mathcal{M} \rightarrow \mathbb{R}^{d}$ is the velocity field and $p: \mathbb{R} \times \mathcal{M} \rightarrow \mathbb{R}$ is the pressure field. For now, we shall only interpret the system (1.1) at the formal level, ignoring issues of regularity or decay, and also ignoring all cohomology by assuming that closed forms are automatically exact; we will return to these issues later, when we discuss local existence theory.

It will be convenient in this paper to use the language of differential geometry, in order to minimise the reliance on the Euclidean metric $\eta$ on $\mathcal{M}$; this will become useful later when we exploit the properties of the Lie derivative $\mathcal{L}_{u}$ (which will not preserve the Euclidean metric in general), as well as when we temporarily switch over to (modified) cylindrical coordinates in Section 9. See for instance [2] for a basic introduction to the differential geometry concepts used in this paper.

We begin with setting out notation for the standard Cartesian coordinates on $\mathcal{M}$, though we emphasise that the differential geometry constructions introduced here are coordinate-independent (although some of them will rely on the standard volume form $d$ vol on $\mathcal{M}$ ). We let $x^{1}, \ldots, x^{d}$ denote $^{2}$ the usual coordinates on $\mathcal{M}=\mathbb{R}^{m} \times$ $(\mathbb{R} / \mathbb{Z})^{d-m}$ (thus $x^{1}, \ldots, x^{m} \in \mathbb{R}$ and $x^{m+1}, \ldots, x^{d} \in \mathbb{R} / \mathbb{Z}$ ). Taking differentials, we obtain the standard 1 -forms $d x^{1}, \ldots, d x^{d}$ on $\mathcal{M}$, and then on taking wedge products we obtain the standard volume form

$$
d \text { vol : }=d x^{1} \wedge \cdots \wedge d x^{d} .
$$

Dually, we have the standard vector fields

$$
\frac{d}{d x^{1}}, \ldots, \frac{d}{d x^{d}}
$$

\footnotetext{
${ }^{1}$ A substantial portion of the discussion here could in fact be extended to arbitrary smooth Riemannian manifold domains, but we will not need to do so here.

${ }^{2}$ We use superscripts here instead of the more customary subscripts $x_{1}, \ldots, x_{d}$ in order to be compatible with the raising and lowering conventions of differential geometry.
} 
We have suggestively written these vector fields to resemble first-order differential operators, but in order to reduce confusion, we will use the symbol $\partial_{i}$ (as opposed to $\frac{\partial}{\partial x^{i}}$ ) for $i=1, \ldots, d$ to denote the partial differentiation operation in the $x^{i}$ direction, to distinguish this partial differentiation operation from the associated vector field $\frac{d}{d x^{i}}$.

We let $\Lambda_{0}(\mathcal{M})$ denote the space of (formal) scalar functions from $\mathcal{M}$ to $\mathbb{R}$. More generally, for any $k \geqslant 0$, let $\Lambda_{k}(\mathcal{M})=\Gamma\left(\bigwedge^{k} T^{*} \mathcal{M}\right)$ denote the space of (formal) $k$-forms on $\mathcal{M}$, thus for instance $d x^{1}, \ldots, d x^{d} \in \Lambda_{1}(\mathcal{M})$ and $d$ vol $\in \Lambda_{d}(\mathcal{M})$. The coordinates of a $k$-form $\omega \in \Lambda_{k}(\mathcal{M})$ will be denoted $\omega_{i_{1} \ldots i_{k}}$, where the indices $i_{1}, \ldots, i_{k}$ range from 1 to $d$ (with the usual summation conventions), and $\omega_{i_{1} \ldots i_{k}}$ is antisymmetric in $i_{1}, \ldots, i_{k}$. Of course, $\Lambda_{k}(\mathcal{M})$ is trivial for $k>d$. The standard basis for $\Lambda_{k}(\mathcal{M})$ (as a $\Lambda_{0}(\mathcal{M})$-module) is given by the constant $k$-forms

$$
d x^{i_{1}} \wedge \cdots \wedge d x^{i_{k}}
$$

for $1 \leqslant i_{1}<\cdots<i_{k} \leqslant d$; thus with the usual summation conventions we have

$$
\omega=\frac{1}{k !} \omega_{i_{1} \ldots i_{k}} d x^{i_{1}} \wedge \cdots \wedge d x^{i_{k}}
$$

(the $\frac{1}{k !}$ factor reflecting the fact that the $i_{1}, \ldots, i_{k}$ are not necessarily in increasing order with the usual summation conventions). Dual to the space $\Lambda_{k}(\mathcal{M})$ of $k$-forms is the space $\Gamma^{k}(\mathcal{M})=\Gamma\left(\bigwedge^{k} T \mathcal{M}\right)$ of (formal) $k$-vector fields on $\mathcal{M}$; the coordinates of an element $\alpha \in \Gamma^{k}(\mathcal{M})$ will be denoted $\alpha^{i_{1} \ldots i_{k}}$ and is antisymmetric in the $i_{1}, \ldots, i_{k}$; again, $\Gamma^{k}(\mathcal{M})$ is trivial for $k>d$, and we adopt the convention that it is trivial for $k<0$ also. We also make the identification $\Lambda_{0}(\mathcal{M}) \equiv \Gamma^{0}(\mathcal{M})$. The standard basis for $\Gamma^{k}(\mathcal{M})\left(\right.$ as a $\Lambda_{0}(\mathcal{M})=\Gamma^{0}(\mathcal{M})$-module) is given by the constant $k$-vector fields

$$
\frac{d}{d x^{i_{1}}} \wedge \cdots \wedge \frac{d}{d x^{i_{k}}}
$$

for $1 \leqslant i_{1}<\cdots<i_{k} \leqslant d$, thus

$$
\alpha=\frac{1}{k !} \alpha^{i_{1} \ldots i_{k}} \frac{d}{d x^{i_{1}}} \wedge \cdots \wedge \frac{d}{d x^{i_{k}}} .
$$

We have the usual pairing operation $\langle\rangle:, \Lambda_{k}(\mathcal{M}) \times \Gamma^{k}(\mathcal{M}) \rightarrow \Lambda_{0}(\mathcal{M})$, defined in coordinates as

$$
\langle\omega, \alpha\rangle:=\frac{1}{k !} \omega_{i_{1} \ldots i_{k}} \alpha^{i_{1} \ldots i_{k}}
$$

thus for instance $\left\langle d x^{i}, \frac{d}{d x^{j}}\right\rangle$ equals 1 when $i=j$, and 0 otherwise.

We have the usual exterior derivative operator $d: \Lambda_{k}(\mathcal{M}) \rightarrow \Lambda_{k+1}(\mathcal{M})$, defined in coordinates as

$$
(d \omega)_{i_{1} \ldots i_{k+1}}:=\sum_{j=1}^{k+1}(-1)^{j-1} \partial_{i_{j}} \omega_{i_{1} \ldots i_{j-1} i_{j+1} \ldots i_{k+1}} ;
$$


this is of course compatible with our notation $d x^{1}, \ldots, d x^{d}$ for the standard 1-forms (viewing each coordinate function $x^{i}$, locally at least, as an element of $\Lambda_{0}(\mathcal{M})$ ). Dually, we have the codifferential ${ }^{3} \delta: \Gamma^{k+1}(\mathcal{M}) \rightarrow \Gamma^{k}(\mathcal{M})$ defined in coordinates as

$$
(\delta \alpha)^{i_{2} \ldots i_{k+1}}:=-\partial_{i_{1}} \alpha^{i_{1} \ldots i_{k+1}} .
$$

Thus, for instance, if $X \in \Gamma^{1}(\mathcal{M})$ is a vector field, then $\delta X=-\operatorname{div} X$ is the negative divergence of $X$. As is well known, we have

$$
d^{2}=0
$$

and

$$
\delta^{2}=0
$$

see e.g. [2, §5.17]. We let $B_{k}(\mathcal{M}):=\left\{\omega \in \Lambda_{k}(\mathcal{M}): d \omega=0\right\}$ denote the space of closed $k$-forms, and similarly let $B^{k}(\mathcal{M}):=\left\{\alpha \in \Gamma^{k}(\mathcal{M}): \delta \alpha=0\right\}$ denote the space of divergence-free $k$-vector fields.

The Euclidean metric $\eta$ on $\mathcal{M}$ is given by its first fundamental form

$$
d \eta^{2}=\left(d x^{1}\right)^{2}+\cdots+\left(d x^{d}\right)^{2} .
$$

It can be viewed in coordinates as a $(0,2)$-tensor $\eta_{i j}$, or after inversion as a $(2,0)$ tensor $\eta^{i j}$. It provides an identification $\tilde{\eta}: \Gamma^{k}(\mathcal{M}) \rightarrow \Lambda_{k}(\mathcal{M})$ of $k$-vector fields with $k$-forms, defined in coordinates by

$$
(\tilde{\eta} T)_{i_{1} \ldots i_{k}}:=\eta_{i_{1} j_{1}} \ldots \eta_{i_{k} j_{k}} T^{j_{1} \ldots j_{k}}
$$

thus for instance

$$
\tilde{\eta}\left(\frac{d}{d x^{i_{1}}} \wedge \cdots \wedge \frac{d}{d x^{i_{k}}}\right)=d x^{i_{1}} \wedge \cdots \wedge d x^{i_{k}}
$$

or upon inverting

$$
\tilde{\eta}^{-1}\left(d x^{i_{1}} \wedge \cdots \wedge d x^{i_{k}}\right)=\frac{d}{d x^{i_{1}}} \wedge \cdots \wedge \frac{d}{d x^{i_{k}}} .
$$

Suppose that $u, p$ (formally) solve (1.1). For each time $t, u(t)$ and $p(t)$ can be viewed as elements of $B^{1}(\mathcal{M})$ and $\Lambda_{0}(\mathcal{M})$ respectively; in coordinates with the usual summation conventions, (1.1) becomes

$$
\partial_{t} u^{i}+u^{j} \partial_{j} u^{i}=-\eta^{i j} \partial_{j} p
$$

\footnotetext{
3 In the usual Hodge theory literature, one uses a Riemannian metric to identify $\Gamma^{k}$ with $\Lambda_{k}$ as per (1.4), so that the codifferential acts on $k$-forms rather than $k$-vector fields. However, it will be more convenient here to avoid using the metric identification to define the codifferential, because the Euclidean metric $\eta$ will not in general be preserved by flowing along the velocity field $u$.
} 


$$
\partial_{i} u^{i}=0
$$

If we define the covelocity $v(t) \in \Lambda_{1}\left(\mathbb{R}^{d}\right)$ to be the 1 -form $v=\tilde{\eta} u$, thus in coordinates

$$
v_{i}:=\eta_{i j} u^{j}
$$

then we see that

$$
\partial_{t} v_{i}+u^{j} \partial_{j} v_{i}+\left(\partial_{i} u^{j}\right) v_{j}=-\partial_{i} \tilde{p}
$$

where the modified pressure $\tilde{p}(t) \in \Lambda_{0}(\mathcal{M})$ is given by the formula

$$
\tilde{p}:=p-\frac{1}{2} u^{j} v_{j}
$$

Recalling (see e.g. [2, §3.4]) that the Lie derivative $\mathcal{L}_{u}$ along a vector field $u$ acts on $k$-forms $\omega \in \Lambda_{k}(\mathcal{M})$ by the Cartan formula

$$
\mathcal{L}_{u} \omega=\iota_{u}(d \omega)+d\left(\iota_{u} \omega\right)
$$

where $\iota_{u}: \Lambda_{k+1}(\mathcal{M}) \rightarrow \Lambda_{k}(\mathcal{M})$ is the contraction operator

$$
\left(\iota_{u} \omega\right)_{i_{2} \ldots i_{k+1}}:=u^{i_{1}} \omega_{i_{1} \ldots i_{k+1}},
$$

we see that

$$
\left(\mathcal{L}_{u} v\right)_{i}=u^{j} \partial_{j} v_{i}+\left(\partial_{i} u^{j}\right) v_{j}
$$

and hence (1.5) can be written in coordinate-free notation as

$$
\partial_{t} v+\mathcal{L}_{u} v=-d \tilde{p}
$$

If we define the vorticity $\omega(t) \in \Lambda_{2}(\mathcal{M})$ to be the exterior derivative

$$
\omega:=d v=d \tilde{\eta} u
$$

of the covelocity $v$, and use the basic commutativity identity

$$
d \mathcal{L}_{u}=\mathcal{L}_{u} d
$$

(see e.g. [2, Proposition 3.6]) and (1.2), we conclude that $\omega(t)$ in fact lies in $B_{2}(\mathcal{M})$ (i.e. it is closed) and obeys the vorticity equation

$$
\partial_{t} \omega+\mathcal{L}_{u} \omega=0
$$


Remark 1.1 The standard volume form $d$ vol $:=d x^{1} \wedge \cdots \wedge d x^{d} \in \Gamma^{d}(\mathcal{M})$, induces the Hodge duality operator $*: \Gamma^{k}(\mathcal{M}) \rightarrow \Lambda_{d-k}(\mathcal{M})$ for $0 \leqslant d \leqslant k$, defined by the formula

$$
\omega \wedge(* \alpha)=\langle\omega, \alpha\rangle d \mathrm{vol}
$$

for $\omega \in \Lambda_{k}(\mathcal{M})$ and $\alpha \in \Gamma^{k}(\mathcal{M})$. Thus for instance we have

$$
\delta=* d *^{-1} .
$$

The dual $*^{-1} \omega \in \Gamma^{d-2}(\mathcal{M})$ of the vorticity is then a scalar function in two dimensions and a vector field in three dimensions, and in the Euler equation literature it is common to refer to this scalar or vector field, rather than the 2-form $\omega$, as the vorticity (i.e. one replaces exterior derivative $d$ with a suitable curl operator). The vorticity equation then becomes the familiar equation $\partial_{t} \omega+(u \cdot \nabla) \omega=0$ (in the two-dimensional case) or $\partial_{t} \omega+(u \cdot \nabla) \omega=(\omega \cdot \nabla) u$ (in the three-dimensional case). However, we will adopt a more differential-geometric viewpoint in this paper by interpreting the vorticity as a 2 -form rather than a scalar or vector field. This distinction becomes particularly important when applying Lie derivatives such as $\mathcal{L}_{u}$, as these derivatives act on 2forms in a different fashion than on scalars or vector fields (this is related to the fact that the velocity field $u$ will almost never be a Killing vector field for the Euclidean metric $\eta$, so that $\mathcal{L}_{u} \eta \neq 0$ ). Interpreting the vorticity as a 2 -form will also make it easier to change to curvilinear coordinate systems, such as cylindrical coordinates, as we will do in Section 9.

The velocity field $u$ can be (formally) recovered from the vorticity $\omega$ by the BiotSavart law

$$
u=\delta \tilde{\eta}^{-1} \Delta^{-1} \omega
$$

where the Hodge Laplacian $\Delta: \Lambda_{k}(\mathcal{M}) \rightarrow \Lambda_{k}(\mathcal{M})$ is given by

$$
\Delta:=d \tilde{\eta} \delta \tilde{\eta}^{-1}+\tilde{\eta} \delta \tilde{\eta}^{-1} d,
$$

which in the Euclidean metric coordinates simplifies to the familiar formula ${ }^{4} \Delta=$ $-\eta^{i j} \partial_{i} \partial_{j}$. Note that $\Delta$ preserves $B_{k}(\mathcal{M})$, and so the inverse operator $\Delta^{-1}$ does so also (formally, at least). We make the technical remark that when $m=2$, the operator $\Delta^{-1}$ is only well defined up to constants, even when applied to forms that are smooth and compactly supported, unless one arbitrarily fixes a convention for defining $\Delta^{-1}$. However this will not be a major issue in practice because the operator $\delta \tilde{\eta}^{-1} \Delta^{-1}$ will remain canonically defined.

\footnotetext{
4 Note here the negative sign in our definition of the Laplacian, which differs from the usual conventions for the Laplacian in the Euler equation literature. In particular, our Laplacian $\Delta$ will be positive semi-definite rather than negative semi-definite. In the differential geometry literature it is common to refer to $\tilde{\eta} \delta \tilde{\eta}^{-1}$ rather than $\delta$ as the codifferential, so that $\Delta=d \delta+\delta d$ in this notation; however we prefer in this paper to make the dependence on the metric $\eta$ more explicit.
} 
By slight abuse of notation, we refer to the 2-vector field $\tilde{\eta}^{-1} \Delta^{-1} \omega(t)$ as the vector potential (also known as the stream function in the two-dimensional case $d=2$ ), and refer to the operator $\tilde{\eta}^{-1} \Delta^{-1}: B_{2}(\mathcal{M}) \rightarrow \Gamma^{2}(\mathcal{M})$ as the vector potential operator for the Euler equations. We observe that the vector potential operator $\tilde{\eta}^{-1} \Delta^{-1}$ is formally self-adjoint in the sense that we have the (formal) integration by parts identity

$$
\int_{\mathbb{R}^{d}}\left\langle\omega, \tilde{\eta}^{-1} \Delta^{-1} \omega^{\prime}\right\rangle d \mathrm{vol}=\int_{\mathbb{R}^{d}}\left\langle\omega^{\prime}, \tilde{\eta}^{-1} \Delta^{-1} \omega\right\rangle d \mathrm{vol}
$$

for $\omega, \omega^{\prime} \in B_{2}(\mathcal{M})$.

We refer to the system

$$
\begin{aligned}
\partial_{t} \omega+\mathcal{L}_{u} \omega & =0 \\
u & =\delta \tilde{\eta}^{-1} \Delta^{-1} \omega
\end{aligned}
$$

as the vorticity-vector potential formulation of the Euler equations. We now generalise this system to other choices of vector potential operator:

Definition 1.2 (Generalised Euler equations). Let $\mathcal{M}=\mathbb{R}^{m} \times(\mathbb{R} / \mathbb{Z})^{d-m}$ for some $d \geqslant 2$ and $0 \leqslant m \leqslant d$, and let $A: B_{2}(\mathcal{M}) \rightarrow \Gamma^{2}(\mathcal{M})$ be a (formal) linear operator from the space of closed 2-forms to the space of 2-vector fields. We refer to the (formal) system of equations

$$
\begin{aligned}
\partial_{t} \omega+\mathcal{L}_{u} \omega & =0 \\
u & =\delta A \omega,
\end{aligned}
$$

where $\omega(t) \in B_{2}(\mathcal{M})$ and $u(t) \in B^{1}(\mathcal{M})$ for each time $t$, as the generalised Euler equations with vector potential operator $A$. We say that the vector potential operator $A$ is formally self-adjoint if one formally has

$$
\int_{\mathcal{M}}\left\langle\omega, A \omega^{\prime}\right\rangle d \mathrm{vol}=\int_{\mathcal{M}}\left\langle\omega^{\prime}, A \omega\right\rangle d \mathrm{vol}
$$

for all $\omega, \omega^{\prime} \in B_{2}(\mathcal{M})$.

The vorticity-vector potential formulation of the Euler equations (which we will now call the true Euler equations for emphasis) are thus the generalised Euler equations associated to the vector potential operator

$$
\tilde{\eta}^{-1} \Delta^{-1}
$$

Another example of a system that can be (formally) written as the generalised Euler equation is the (inviscid) surface quasi-geostrophic ( $S Q G)$ equations

$$
\begin{aligned}
\partial_{t} \theta+(u \cdot \nabla) \theta & =0 \\
u & =\left(-\partial_{2} \Delta^{-1 / 2}, \partial_{1} \Delta^{-1 / 2}\right) \theta
\end{aligned}
$$


in two spatial dimensions $d=2$, where $\theta: \mathbb{R} \times \mathcal{M} \rightarrow \mathbb{R}$ is a scalar field and $u: \mathbb{R} \times \mathcal{M} \rightarrow \mathbb{R}^{2}$ is a vector field. This equation arises in atmospheric science and can be considered as a toy model for the three-dimensional Euler equations; see [24] for further discussion. If we set

$$
\omega:=\theta d \text { vol }=\theta d x_{1} \wedge d x_{2}
$$

and define the vector potential operator $A: B_{2}(\mathcal{M}) \rightarrow \Gamma^{2}(\mathcal{M})$ by $A:=\Delta^{-1 / 2}$, or in coordinates

$$
A\left(\theta d x^{1} \wedge d x^{2}\right):=\Delta^{-1 / 2} \theta \frac{d}{d x^{1}} \wedge \frac{d}{d x^{2}}
$$

then we see that the SQG equations become the generalised Euler equations in two dimensions with the choice (1.14) of vector potential operator. Later, in Section 8.1, we will give an alternate way of interpreting SQG as a generalised Euler equation, this time in three dimensions, and with a vector potential operator of order -2 (like $\left.\tilde{\eta}^{-1} \Delta^{-1}\right)$.

Remark 1.3 The modified $S Q G$ equations, in which the exponent $-1 / 2$ appearing in (1.14) is replaced by $-\alpha / 2$ for some parameter $\alpha$ between 1 and 2 , is a family of interpolants between SQG and the two-dimensional Euler equations which have also been studied in the literature; see e.g. [49]. However, we will not study these equations further in this paper, though we will note the recent paper [51] in which finite time blowup was established for patch solutions to the generalised SQG equations in a half-plane.

Remark 1.4 The formalism in Definition 1.2 does not directly use the Euclidean metric $\eta$ on $\mathcal{M}$; one only needs the structure $(\mathcal{M}, d$ vol) of $\mathcal{M}$ as a smooth manifold equipped with a volume form $d$ vol (in order to define the codifferential $\delta$ ). However, when one works with the true Euler equations, the Euclidean metric $\eta$ is needed to define the vector potential operator $A=\tilde{\eta}^{-1} \Delta^{-1}$. Thus we see that the role of Euclidean geometry (beyond the volume form) in the true Euler equations has been completely captured in this formalism by the operator $A$.

Remark 1.5 One can rewrite the generalised Euler equations in a form resembling the traditional form (1.1) of the true Euler equations by formally defining the covelocity $v \in \Lambda_{1}(\mathcal{M})$ to solve the system

$$
d v=\omega ; \quad \delta \tilde{\eta}^{-1} v=0
$$

and then the generalised Euler equations may be rewritten as

$$
\begin{aligned}
\partial_{t} v+\mathcal{L}_{u} v & =d \tilde{p} \\
u & =\delta \tilde{\eta}^{-1} A d v \\
\delta \tilde{\eta}^{-1} v & =0 .
\end{aligned}
$$


The generalised Euler equations (formally) obey many of the conservation laws that the true Euler equations do, particularly if the vector potential operator $A$ is formally self-adjoint and commutes with a suitable symmetry. More precisely, we have

Proposition 1.6 (Formal conservation laws). Let $\mathcal{M}=\mathbb{R}^{m} \times(\mathbb{R} / \mathbb{Z})^{d-m}$ for some $d \geqslant 2$ and $0 \leqslant m \leqslant d$, and let $A: B_{2}(\mathcal{M}) \rightarrow \Gamma^{2}(\mathcal{M})$ be a (formal) linear operator. Let $\omega, u$ solve the generalised Euler equations with vector potential operator $A$.

(i) (Kelvin circulation theorem) If $S=S(t)$ is a (time-dependent, oriented) surface with boundary that evolves along the (time-dependent) velocity field $u=u(t)$, then the quantity ${ }^{5} \int_{S} \omega$ is formally conserved in time.

(ii) (Preservation of vortex streamlines) If $d=3$, then the curves formed by integrating the vector field $*^{-1} \omega(t) \in \Gamma^{1}(\mathcal{M})$ (i.e., the vortex streamlines) are transported by the velocity field $u$.

(iii) (Conservation of helicity) If $\mathcal{M}=\mathbb{R}^{3}$, define the helicity $H(t)$ to be the quantity $H(t):=\int_{\mathcal{M}} v(t) \wedge \omega(t)$, where $v(t) \in \Lambda_{1}(\mathcal{M})$ is an arbitrary 1-form with $d v=\omega$; observe from Stokes' theorem that this quantity does not depend on the choice of $v$. Then $H$ is formally conserved in time.

(iv) (Conservation of Hamiltonian) Suppose A is formally self-adjoint. Define the energy $E(t)$ to be the quantity

$$
E(t):=\frac{1}{2} \int_{\mathcal{M}}\langle\omega, A \omega\rangle d \mathrm{vol} .
$$

Then $E$ is formally conserved in time.

(v) (Conservation of impulse) Suppose A is formally self-adjoint. Let $X \in B^{1}(\mathcal{M})$ be a (time-independent) divergence-free vector field such that the Lie derivative $\mathcal{L}_{X}$ commutes with $A: \mathcal{L}_{X} A=A \mathcal{L}_{X}$. Suppose that $\alpha \in \Gamma^{2}(\mathcal{M})$ is a timeindependent 2-vector field such that $\delta \alpha=X$. Then the quantity $\int_{\mathcal{M}}\langle\omega, \alpha\rangle d$ vol is formally conserved in time.

In the case of the true Euler equations with $\mathcal{M}=\mathbb{R}^{d}$, examples of (formal) conservation laws arising from Proposition $8.1(\mathrm{v})$ include the total vorticity

$$
\Omega_{i j}:=\int_{\mathcal{M}} \omega_{i j} d \mathrm{vol}
$$

(corresponding to the zero vector field), the impulse

$$
I_{j}:=\frac{-1}{d-1} \int_{\mathcal{M}} x^{i} \omega_{i j} d \mathrm{vol}
$$

\footnotetext{
5 In the case of the true Euler equations, this quantity $\int_{S} \omega$ can be expressed via Stokes' theorem as $\int_{\partial S} \tilde{\eta} u$, which is the physical circulation of velocity along the boundary $\partial S$ of $S$. For the generalised Euler equations, this quantity is not quite the physical circulation, but is instead the quantity $\int_{\partial S} v$ where $v$ is the covelocity from Remark 1.5. Nevertheless we shall abuse notation and refer to the quantity $\int_{S} \omega$ as the circulation around the surface $S$. We thank Peter Constantin for pointing out this subtle distinction between the circulation conserved by Kelvin's theorem and physical circulation in the context of generalised Euler equations.
} 
(corresponding to the translation vector fields $\frac{d}{d x^{j}}$ ), and the moment of impulse

$$
M_{j k}:=\frac{-1}{d} \int_{\mathcal{M}} \eta_{l k} x^{i} x^{l} \omega_{i j}-\eta_{l j} x^{i} x^{l} \omega_{i k} d \text { vol }
$$

(corresponding to the rotation vector field $x^{i} \frac{d}{d x^{j}}-x^{j} \frac{d}{d x^{i}}$ ). If the velocity field $u$ has sufficient decay, then $\Omega_{i j}$ vanishes, $I_{j}$ is equal to the total momentum $\int_{\mathcal{M}} u^{j} d$ vol (after contracting by $\eta$ ), and $M_{j k}$ is equal to the total angular momentum $\int_{\mathcal{M}}\left(x^{j} u^{k}-\right.$ $x^{k} u^{j}$ ) $d$ vol (again after contracting by $\eta$ ); however, the Biot-Savart law does not always give enough decay on $u$ to justify these computations, even when $\omega$ is smooth and compactly supported. See $[58,61]$ for further discussion of these conservation laws.

We prove Proposition 1.6 in Section 2 by direct computation, relying mostly on the standard properties of the Lie and exterior derivatives. One can also interpret these conservation laws as instances of Noether's theorem, using the Euler-Poincaré interpretation of generalised Euler equations (in the spirit of [1]) as formal geodesic flow in the infinite-dimensional manifold of volume-preserving diffeomorphisms of $\mathcal{M}$, with the vector potential operator $A$ determining the formal (right-)invariant Riemannian metric to place on this manifold; see Section 3. It is certainly possible to prove rigorous versions of Proposition 1.6 assuming sufficient regularity and decay of the solution (and assuming that there are no cohomological obstructions), but we will not need to do so here (except for Proposition 1.6(i), which is used to prove Theorem 1.11 below).

\subsection{Local Existence Theory}

Thus far, all of our discussion has been purely formal, ignoring all requirements of decay and smoothness. We now turn to the rigorous existence theory of the generalised Euler equations. For this we will need to place the fields $u$ and $\omega$ in appropriate (high regularity) function spaces; we will also now work in coordinates, abandoning any pretense of coordinate invariance. As mentioned previously, we will assume that the number $m$ of non-compact directions is at least two, in order to avoid problems with defining the inverse $\Delta^{-1}$ of the Hodge Laplacian.

For any $1 \leqslant p \leqslant \infty$, we let $L^{p} \cap \Lambda_{k}(\mathcal{M})$ be the space of $k$-forms that are $p^{\text {th }}$ power integrable, with the usual norm

$$
\|\omega\|_{L^{p}(\mathcal{M})}:=\left(\int_{\mathcal{M}}|\omega|^{p} d \mathrm{vol}\right)^{1 / p}
$$

with the usual Euclidean norm on tensors to define $|\omega|$, and with the usual modifications for $p=\infty$. Similarly define $L^{p} \cap \Gamma^{k}(\mathcal{M}), L^{p} \cap B^{k}(\mathcal{M})$, and $L^{p} \cap B_{k}(\mathcal{M})$, where in the latter two cases we interpret the differential operators $d, \delta$ in the distributional sense, thus for instance $L^{p} \cap B^{k}(\mathcal{M})$ consists of those $\alpha$ in $\Gamma^{k}(\mathcal{M})$ that are $p^{\text {th }}$ power integrable with $\delta \alpha=0$ in the sense of distributions. For any $k \geqslant 0$ and $s \in \mathbb{R}$, we define $\dot{H}^{s} \cap \Lambda_{k}(\mathcal{M})$ to be the space of tempered distributional $k$-forms $\omega \in \Lambda_{k}(\mathcal{M})$ whose (tempered distributional) Fourier transform 


$$
\hat{\omega}(\xi):=\int_{\mathcal{M}} \omega(x) e^{-2 \pi i x \cdot \xi} d x
$$

(computed by working in the standard coordinate basis and taking the tempered distributional Fourier transform of each component of $\omega$ separately) is such that $|\xi|^{s} \hat{\omega}$ is square-integrable, thus

$$
\|\omega\|_{\dot{H}^{s}(\mathcal{M})}^{2}:=\int_{\hat{\mathcal{M}}}|2 \pi \xi|^{2 s}|\hat{\omega}(\xi)|^{2} d \xi<\infty
$$

(here we use the standard Euclidean norm on tensors to define $|\hat{\omega}(\xi)|$, and $\hat{\mathcal{M}}=$ $\mathbb{R}^{m} \times \mathbb{Z}^{d-m}$ denotes the Pontryagin dual of $\left.\mathcal{M}\right)$. The factors of $2 \pi$ are of very minor importance and can be ignored for a first reading. The space $\dot{H}^{s} \cap \Lambda_{k}(\mathcal{M})$ can be easily verified to be a Hilbert space. Similarly define $\dot{H}^{s} \cap \Gamma^{k}(\mathcal{M}), \dot{H}^{s} \cap B_{k}(\mathcal{M})$, and $\dot{H}^{s} \cap B^{k}(\mathcal{M})$. As is usual, we write $H^{s}:=L^{2} \cap \dot{H}^{s}$ (thus for instance $H^{s} \cap \Lambda_{k}=$ $\left.L^{2} \cap \dot{H}^{s} \cap \Lambda_{k}\right)$ and

$$
\|\omega\|_{H^{s}(\mathcal{M})}^{2}:=\|\omega\|_{L^{2}(\mathcal{M})}^{2}+\|\omega\|_{\dot{H}^{s}(\mathcal{M})}^{2} .
$$

We also define $C_{c}^{\infty} \cap \Lambda_{k}(\mathcal{M})$ to be the space of $k$-forms that are smooth and compactly supported, and similarly define $C_{c}^{\infty} \cap \Gamma^{k}(\mathcal{M})$, etc..

Fix an integer $s>\frac{d}{2}+1$, and let $1<p \leqslant 2$ be an exponent ${ }^{6}$ with $p<m$, where we recall that $m$ is the number of non-compact dimensions in $\mathcal{M}$. It turns out that a convenient space to place the vorticity field $\omega(t)$ for a given time $t$ is

$$
L^{p} \cap H^{s} \cap B_{2}(\mathcal{M})
$$

More precisely, to construct solutions on the time interval $[0, T]$, we will place $\omega$ in the space

$$
X^{s, p}:=C^{0}\left([0, T] \rightarrow L^{p} \cap H^{s} \cap B_{2}(\mathcal{M})\right) \cap C^{1}\left([0, T] \rightarrow H^{s-1} \cap B_{2}(\mathcal{M})\right),
$$

thus the map $t \mapsto \omega(t)$ will be required to be a continuous map into $L^{p} \cap H^{s} \cap B_{2}(\mathcal{M})$, and a continuously differentiable map into $H^{s-1} \cap B_{2}(\mathcal{M})$, where we of course give $L^{p} \cap H^{s} \cap B_{2}(\mathcal{M})$ the topology generated by the $L^{p}$ and $H^{s}$ norms, and similarly for $H^{s-1} \cap B_{2}(\mathcal{M})$. Note from Sobolev embedding and the hypothesis $s>\frac{d}{2}+1$ that this implies that $\omega \in C_{t, x}^{1}([0, T] \times \mathcal{M})$. Similarly, we will place the velocity field $u$ in the space

$$
Y^{s, p}:=C^{0}\left([0, T] \rightarrow \dot{W}^{1, p} \cap \dot{H}^{s+1} \cap B^{1}(\mathcal{M})\right),
$$

where $\dot{W}^{1, p}$ is the Sobolev space of functions (or vector fields, in this case) whose distributional derivative lies in $L^{p}$; for technical reasons relating to the slow decay of

\footnotetext{
${ }^{6}$ In particular, when $m \geqslant 3$ we can take $p=2$, which simplifies some of the discussion below. On the other hand, these hypotheses are not satisfiable if $m=0$ or $m=1$. The reason we need the $L^{p}$ integrability for the vorticity $\omega$ is in order to make sense of the velocity $u=\delta A \omega$ as a continuous function, and not merely as a distribution.
} 
the Biot-Savart law (and its generalisations) at infinity, we do not insist that $u$ itself lies in $L^{2}$ or $L^{p}$. Note that the hypothesis $u \in Y^{s, p}$ and Sobolev embedding ${ }^{7}$ implies that $u \in C_{t}^{0} C_{x}^{1}([0, T] \times \mathcal{M})$. This is sufficient regularity to interpret the equation (1.10) in the classical sense, as a "strong" solution rather than merely a weak distributional solution.

To interpret (1.11), we will of course need some regularity hypotheses on the operator $A: B_{2}(\mathcal{M}) \rightarrow \Gamma^{2}(\mathcal{M})$. We will adopt the following choice of hypotheses. We use $X \lesssim Y$ or $X=O(Y)$ to denote the estimate $|X| \leqslant C Y$, where $C$ is a constant; if $C$ is to depend on one or more parameters, we indicate this by subscripting the $\lesssim$ or $O()$ notation appropriately.

Definition 1.7 (Reasonable operator). Let $M$ be a natural number, and let $\mathcal{M}=$ $\mathbb{R}^{m} \times(\mathbb{R} / \mathbb{Z})^{d-m}$ for some $2 \leqslant m \leqslant d$. A vector potential operator $A: C_{c}^{\infty} \cap B_{2}(\mathcal{M}) \rightarrow$ $\Gamma^{2}(\mathcal{M})$ is said to be $M$-reasonable if it has an integral representation

$$
A \omega(x)=\int_{\mathcal{M}} K(x, y) \omega(y) d \operatorname{vol}(y)
$$

where the (tensor-valued) kernel $K$ is smooth for $x \neq y$ and obeys the estimates

$$
\left|\nabla_{x}^{i} \nabla_{y}^{j} K(x, y)\right| \lesssim d, M, A \max \left(|x-y|^{-i-j-d+2},|x-y|^{-i-j-m+2}\right)
$$

for all $x \neq y$ and all $0 \leqslant i, j \leqslant M$ with $i+j \geqslant 1$, where $|x-y|$ denotes the distance between $x$ and $y$ in $\mathcal{M}$ with respect to the Euclidean metric $\eta$; furthermore we assume that

$$
\left\|\nabla^{2} A \omega\right\|_{H^{k}(\mathcal{M})} \lesssim d, M, A\|\omega\|_{H^{k}(\mathcal{M})}
$$

for all $0 \leqslant k \leqslant M$ and all $\omega \in C_{c}^{\infty} \cap B_{2}(\mathcal{M})$. In particular, $\delta A$ can be continuously extended to a map from $L^{2} \cap B_{2}(\mathcal{M})$ to $\dot{H}^{1} \cap \Gamma^{1}(\mathcal{M})$.

Remark 1.8 The right-hand side of (1.19) has the geometric interpretation of being comparable to $\frac{1}{|x-y|^{i+j-2} \operatorname{vol}\left(B_{\mathcal{M}}(0,|x-y|)\right)}$, where $\operatorname{vol}\left(B_{\mathcal{M}}(0,|x-y|)\right)$ is the volume of the ball in $\mathcal{M}$ centred at the origin with radius $|x-y|$. In particular, the operator $\nabla^{2} A$ is a singular integral operator whose kernel obeys estimates of Calderón-Zygmund type, which is of course consistent with the hypothesis (1.20).

From Plancherel's theorem and the fundamental solution for the Laplacian on $\mathcal{M}$, we see that the vector potential operator $\tilde{\eta}^{-1} \Delta^{-1}$ associated to the true Euler equations obeys these requirements whenever $m \geqslant 2$. On the other hand, the vector potential operator (1.14) associated to the SQG equations do not, as in this case $A$ is only smoothing of order 1 rather than 2. With the assumption that $A$ is $M$-reasonable for some sufficiently large $M$, one can now interpret (1.11) rigorously when $\omega \in X$ and $u \in Y$.

\footnotetext{
7 More precisely, observe from Bernstein's inequality and the hypothesis $p<m$ that $\dot{W}^{1, p}$ embeds into $C_{x}^{1}$ at low frequencies, and $\dot{H}^{s+1}$ embeds into $C_{x}^{1}$ at high frequencies.
} 
Using mostly standard "quasilinear well-posedness" energy methods (following the basic approach of Bona and Smith [5], as described in the survey [70]), we can prove the following classical local existence theorem:

Theorem 1.9 (Local existence). Let $\mathcal{M}=\mathbb{R}^{m} \times(\mathbb{R} / \mathbb{Z})^{d-m}$ for some $d \geqslant 2$ and $0 \leqslant m \leqslant d$. Let $s>\frac{d}{2}+1$ be an integer, let $1<p \leqslant 2$ be such that $p<m$, and let $A: C_{c}^{\infty} \cap B_{2}(\mathcal{M}) \rightarrow \dot{H}^{1} \cap \Gamma^{2}(\mathcal{M})$ be a $s+1$-reasonable vector potential operator. Then for any $M>0$ there exists $T>0$ such that for any $\omega_{0} \in L^{p} \cap H^{s} \cap B_{2}(\mathcal{M})$ with $\left\|\omega_{0}\right\|_{L^{p}(\mathcal{M})}+\left\|\omega_{0}\right\|_{H^{s}(\mathcal{M})}<M$, there exists a unique classical solution $\omega \in$ $X^{s, p}$ and $u \in Y^{s, p}$ (with $X^{s, p}, Y^{s, p}$ defined in (1.16), (1.17) respectively) obeying the generalised Euler equations (1.10), (1.11). Furthermore the solution $\omega$ depends continuously on $\omega_{0}$ in the indicated topologies.

Finally, we have the Beale-Kato-Majda blowup criterion [3]: if the solution constructed above cannot be continued beyond a time $0<T_{*}<\infty$ in the indicated function spaces, then

$$
\int_{0}^{T_{*}}\|\omega(t)\|_{L^{\infty}(\mathcal{M})} d t=\infty
$$

We prove this theorem in Section 4. The argument is straightforward when $m \geqslant 3$, in which case the $L^{p}$ norm plays no essential role. However, the situation becomes delicate in the $m=2$ case, basically because the generalised Biot-Savart operator $\delta A$ that appears in the vorticity-vector potential formulation no longer maps $H^{s}$ into $L^{\infty}$ at low frequencies, and one must take advantage of the $L^{p}$ norm and Littlewood-Paley decomposition to close the argument. A slightly different energy method approach to these equations is also given in [58, Chapter 3]. There is also a particle trajectory method to construct solutions to the true Euler equations using the contraction mapping theorem rather than quasilinear method; see e.g. [58, Chapter 4]. However, we were unable to extend it to this general context unless one imposed a translationinvariance hypothesis on the vector potential operator $A$, as the estimates required for the contraction mapping theorem appeared to fail if this hypothesis was not enforced. It may also be possible to extend the local existence arguments in [37] for the true Euler equations, based on the aforementioned interpretation of these equations as a geodesic flow, to the setting of the generalised Euler equations.

Remark 1.10 There are several refinements of the Beale-Kato-Majda blowup criterion in the literature $[9,21,34,60]$. It seems likely to the author that analogues of at least some of these criteria can also be established for the generalised Euler equations (since the generalised Biot-Savart law obeys very similar estimates to the true Biot-Savart law), although we have not attempted to do so here.

\subsection{Finite Time Blowup}

We now turn to the main focus of this paper, namely the establishment of finite time blowup results for generalised Euler equations. 
It is a notorious open problem as to whether smooth solutions to the threedimensional true Euler equations (with suitable decay at infinity) can be extended globally in time, although it is widely expected that finite time blowup can occur for this system; see for instance the surveys $[11,20]$ and recent numerical evidence for blowup in [56,57], as well as a proposed blowup mechanism in [6].

As the global regularity problem for the true Euler equations is difficult to resolve directly, there have been a number of studies of more tractable models of the Euler equations. In particular, finite time blowup has been established for a number of equations that capture some, though not all, of the features of the true Euler-type equations. For instance:

- In [46] a dyadic "shell model" of the Euler equations was introduced, and shown to have solutions that blow up in finite time; see also [64] for a variant of this construction that allows for Navier-Stokes type dissipation. These shell models have the same scaling features as the true Euler equations in three dimensions, as well as energy conservation, but do not have the vorticity transport equation.

- In [23], a one dimensional model for the vorticity equation of the true Euler equations was introduced, and again shown to have solutions that blow up in finite time; see also the later papers $[32,33,59,74]$ for further analysis of this model and its variants. These equations capture many of the features of the Euler equations, such as energy conservation, vorticity stretching and an Euler-Poincaré Lagrangian formulation, but do not correspond to incompressible flows (the formal Euler-Poincaré geodesic flow is on the space of all diffeomorphisms of a manifold, rather than all volume-preserving diffeomorphisms).

- In $[43,45]$, a model of the axially symmetric true Euler equations with swirl was studied in which the convection term was removed, and solutions constructed that blow up in finite time. This system of equations still conserves energy, but does not appear to have an Euler-Poincaré formulation, or a vorticity transport equation analogous to (1.8).

- Further 1D models of axially symmetric true Euler equations outside of a cylindrical obstacle were studied in $[17,18,43]$, again with a number of finite time blowup results; these systems have some remnant of circulation conservation (through the transport of the "temperature" field $\theta$ ), but do not appear to have an Euler-Poincaré formulation that involves an incompressible flow.

See also $[8,14-16,22,25-28,30,35,36,39-43,47,49,54,61,69]$ for further study of the existence and blowup properties of various simplified models for the Euler and Navier-Stokes equations.

In this paper we establish some finite time blowup results in three spatial dimensions for generalised Euler equations, with reasonable vector potential operator $A$. In order to maximise the resemblance of these generalised Euler equations to the true Euler equations, it is desirable to ensure that $A$ be formally self-adjoint, and for $A$ to furthermore be "positive definite" in the sense that the conserved energy (1.15) to be comparable to $\|\omega\|_{\dot{H}^{-1}}^{2}$ (or to $\|u\|_{L^{2}}^{2}$ ). It would also be desirable to construct blowup solutions that are well localised in space, for instance by requiring the initial vorticity to be compactly supported. Finally, one would like to demonstrate some stability in 
the blowup, by showing that blowup persists under some reasonable perturbation of the initial data.

Unfortunately, we were not able to construct a blowup solution in which all of these desirable criteria were satisfied simultaneously. However, we were able produce three different constructions which enjoyed various subsets of this set of desiderata. Taken together, they suggest that one should not be able to establish global regularity properties for the true Euler equations merely by using properties that are shared with the generalised Euler equations, such as energy conservation, the Kelvin circulation theorem, and function space estimates for the vector potential operator.

Our first construction has compactly supported initial data (and a stable blowup), but a non-self-adjoint (and non-positive definite) vector potential operator $A$ :

Theorem 1.11 (Stable non-self-adjoint blowup). Let $\mathcal{M}=\mathbb{R}^{3}$. Then there exists a 100-reasonable vector potential operator $A: C_{c}^{\infty} \cap B_{2}(\mathcal{M}) \rightarrow \Gamma^{2}(\mathcal{M})$ and initial data $\omega_{0} \in C_{c}^{\infty} \cap B_{2}(\mathcal{M})$ such that there is no solution $\omega \in X^{10,2}, u \in Y^{10,2}$ with initial data $\omega_{0}$ on the time interval $[0,1]$.

We prove this result in Section 7. The exponents 10, 100 here have no particular significance and are chosen primarily for sake of concreteness. The blowup is proba$\mathrm{bly}^{8}$ of a "neck pinch" nature, in which the vortex lines focus at a point (see Figure 1); the non-self-adjoint vector potential $A$ is designed to keep transporting the vorticity ever closer to that point. We will not be able to achieve any fine level of control on the dynamics of this finite time blowup, but fortunately we can use the conservation of circulation, combined with a careful choice of $A$, to evaluate the velocity field $u$ near the blowup point and close the argument. As can be seen from the proof, the blowup in Theorem 1.11 is stable in the sense the initial data $\omega_{0}$ can range in an open set in $C_{c}^{\infty} \cap B_{2}$; any smooth closed perturbation of the data supported in a slight enlargment of the support of $\omega_{0}$ will still lead to a solution that blows up in finite time.

As the initial data $\omega_{0}$ and operator $A$ constructed in Theorem 1.11 are compactly supported in space, it is an easy matter to extend the above theorem to $\mathbb{R}^{2} \times \mathbb{R} / \mathbb{Z}$; by adding compact dummy dimensions one can also extend to the case $d \geqslant 3$ and $2 \leqslant m \leqslant 3$. It is likely that one can in fact obtain a result of the above form for arbitrary $d \geqslant 3$ and $2 \leqslant m \leqslant d$ (increasing the exponents 10 and 100 as necessary), but we will not do so here.

The blowup in Theorem 1.11 is perhaps unsurprising, given that the vector potential operator $A$ was not self-adjoint and so did not even have a conserved energy. Our second blowup result involves a vector potential operator $A$ which is now self-adjoint and positive definite. However, to retain compact support of the data, it becomes convenient to work in the domain $\mathbb{R}^{2} \times \mathbb{R} / \mathbb{Z}$ rather than $\mathbb{R}^{3}$; also, the blowup is less stable, as we require the initial data to be translation-invariant in the $\mathbb{R} / \mathbb{Z}$ direction (in order to reduce matters to a two-dimensional problem).

\footnotetext{
${ }^{8}$ Because our argument will be a proof by contradiction, we will not actually be able to guarantee that the solution blows up as intended; it may blow up at an earlier time than the formation of the neck pinch due to other instabilities in the dynamics. However, the "neck pinch" scenario is what the blowup should be, if it is not pre-empted by some earlier, unforeseen blowup. Similarly for the other finite time blowup results in this paper.
} 


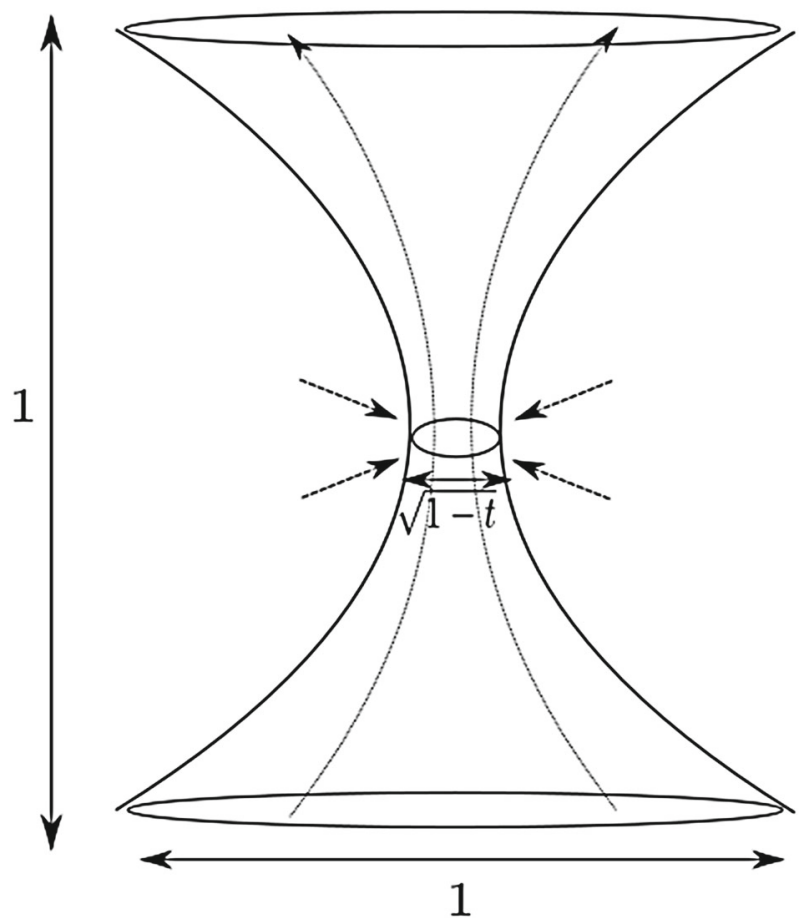

Fig. 1 A schematic depiction of a "neck pinch" blowup of the type expected from the construction in Theorem 1.11 (ignoring a technical parameter $M$ appearing in the proof). At times $t$ close to the blowup time (assumed here to be $T_{*}=1$ ), the vortex streamlines (shown here as dotted curves) travel through a truncated hyperboloid region that is of unit diameter at its ends, but "pinches" through a narrow disk of radius comparable to $\sqrt{1-t}$, so that the vorticity increases to be comparable to $\frac{1}{1-t}$ near this disk, as per the Kelvin circulation theorem. (The vortex streamlines may connect back to themselves outside of this hyperboloid region; this is not pictured in the figure.) The velocity field, depicted here as dashed arrows, points inwards with magnitude comparable to $\frac{1}{\sqrt{1-t}}$ in the pinching region. At time $t=1$, the vorticity becomes infinite at a point, causing blowup

Theorem 1.12 (Self-adjoint partially periodic blowup). Let $\mathcal{M}=\mathbb{R}^{2} \times \mathbb{R} / \mathbb{Z}$ and let $\varepsilon>0$. Then there exists a 100-reasonable, formally self-adjoint vector potential operator $A: C_{c}^{\infty} \cap B_{2}(\mathcal{M}) \rightarrow \Gamma^{2}(\mathcal{M})$ obeying the positive definiteness property

$$
(1-\varepsilon)\|\omega\|_{\dot{H}^{-1}(\mathcal{M})}^{2} \leqslant \int_{\mathcal{M}}\langle\omega, A \omega\rangle d \mathrm{vol} \leqslant(1+\varepsilon)\|\omega\|_{\dot{H}^{-1}(\mathcal{M})}^{2}
$$

for all $\omega \in C_{c}^{\infty} \cap B_{2}(\mathcal{M})$, as well as an initial vorticity $\omega_{0} \in C_{c}^{\infty} \cap B_{2}(\mathcal{M})$ such that there is no solution $\omega \in X^{10,2}, u \in Y^{10,2}$ with initial data $\omega_{0}$ on the time interval $[0,1]$.

We prove this result in Section 8. The main idea is to work in a "two-and-a-halfdimensional" ansatz in which the velocity field $u$ and vorticity $\omega$ are invariant with respect to translations in the $x^{3}$ direction, with the $d x^{1} \wedge d x^{2}$ component of the vorticity vanishing, but in which the third component $u^{3}$ of the velocity is allowed 


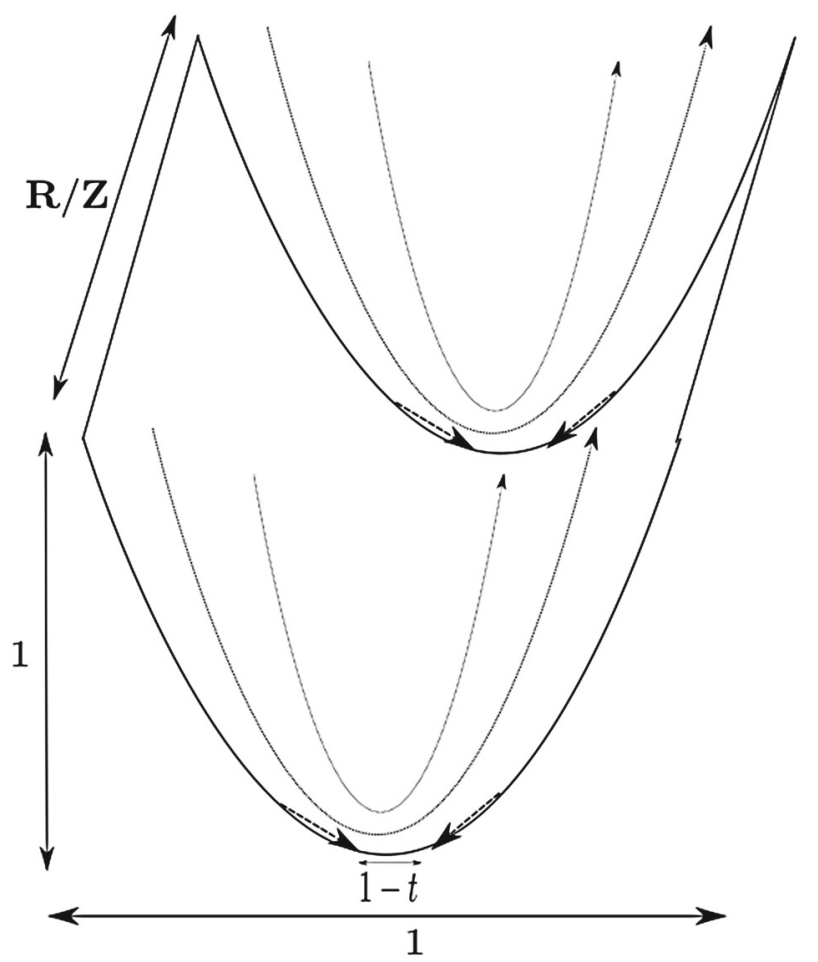

Fig. 2 A schematic depiction of a "two-and-a-half-dimensional" blowup of the type expected from the construction in Theorem 1.12 (again ignoring a technical parameter $M$ ). At times $t$ close to the blowup time (assumed here to be $T_{*}=1$ ), the vortex streamlines (shown here as dotted curves) are horizontal curves that are pinched into a narrow region of horizontal diameter about $1-t$, wherein the vorticity increases to about $\frac{1}{1-t}$ as per the Kelvin circulation theorem. The vorticity is invariant with respect to vertical translations, which in the case of the true Euler equations would mean that the velocity field would be purely vertical (and invariant along streamlines), and the solution would be stationary. Here, we work with a perturbation of the true Euler equations that creates some horizontal velocity in the pinching region (of magnitude comparable to 1 ) that causes the vorticity to pinch further. At time $t=1$, the vorticity becomes infinite on a vertical line (or more precisely, a copy of $\mathbb{R} / \mathbb{Z}$ ), causing blowup

to be non-zero. If the vector potential operator $A$ is chosen properly, it turns out that this component $u^{3}$ obeys an SQG-like active scalar equation on $\mathbb{R}^{2}$; furthermore, the vector potential operator $A_{0}$ for this SQG-like equation is no longer required to be selfadjoint. It is then possible to modify the three-dimensional construction in Theorem 1.11 to create a two-dimensional blowup for this component $u^{3}$, which then implies blowup for the original fields $u, \omega$; in fact the two-dimensional case is a little easier than the three-dimensional one, and is carried out in Section 6. Due to the dimensional reduction, the solution constructed in Theorem 1.12 will (probably) blow up on a one-dimensional set, namely a copy of $\mathbb{R} / \mathbb{Z}$ in $\mathbb{R}^{2} \times \mathbb{R} / \mathbb{Z}$, in contrast to the solution in Theorem 1.11 which (probably) blows up at a point. A schematic depiction of what the blowup should look like in this construction is given in Figure 2.

Finally, we remove the periodic dimension from Theorem 1.12: 


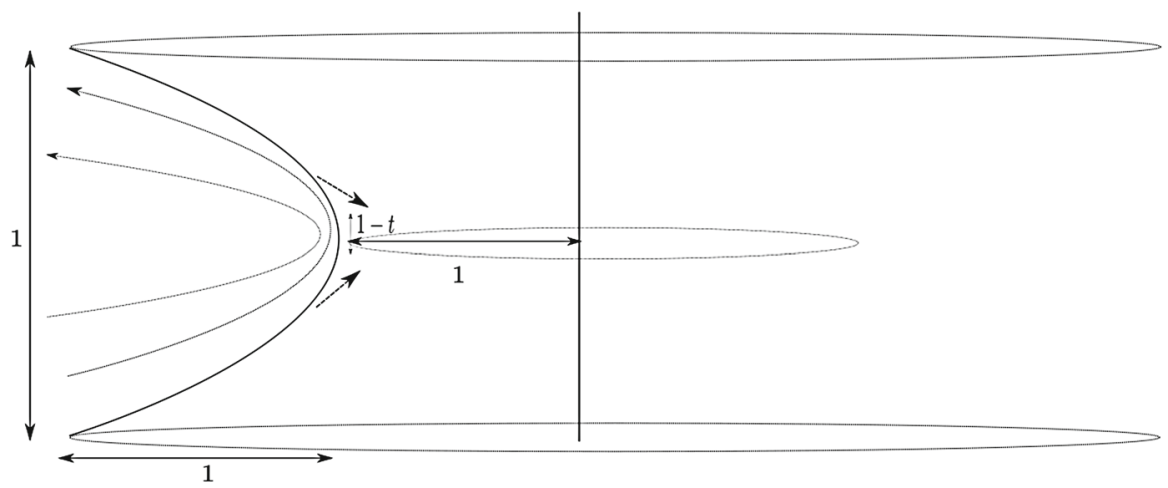

Fig. 3 A schematic depiction of a "axisymmetric with swirl" blowup of the type expected from the construction in Theorem 1.13, which is essentially the same blowup as Theorem 1.12 if the Cartesian coordinates were replaced with cylindrical ones. At times $t$ close to the blowup time (assumed here to be $T_{*}=1$ ), the vortex streamlines (shown here as dotted curves) are curves coplanar with the vertical axis (drawn here as a thick line) that are pinched into a toroidal region that is roughly a $1-t$-neighbourhood of a circle of radius comparable to 1 . Within this region, the vorticity increases to about $\frac{1}{1-t}$ as per the Kelvin circulation theorem. The vorticity and velocity fields are axially symmetric; the latter has magnitude comparable to 1 in the toroidal region, with significant inward components as well as some "swirl" around the vertical axis. At time $t=1$, the vorticity becomes infinite on a circle, causing blowup

Theorem 1.13 (Self-adjoint non-periodic blowup). Let $\mathcal{M}=\mathbb{R}^{3}$ and let $\varepsilon>0$. Then there exists a 100-reasonable, formally self-adjoint vector potential operator $A: C_{c}^{\infty} \cap B_{2}(\mathcal{M}) \rightarrow \Gamma^{2}(\mathcal{M})$ obeying the positive definiteness property

$$
(1-\varepsilon)\|\omega\|_{\dot{H}^{-1}(\mathcal{M})}^{2} \leqslant \int_{\mathcal{M}}\langle\omega, A \omega\rangle d \operatorname{vol} \leqslant(1+\varepsilon)\|\omega\|_{\dot{H}^{-1}(\mathcal{M})}^{2}
$$

for all $\omega \in C_{c}^{\infty} \cap B_{2}(\mathcal{M})$, as well as an initial vorticity $\omega_{0} \in C_{c}^{\infty} \cap B_{2}(\mathcal{M})$ such that there is no solution $\omega \in X^{10,2}, u \in Y^{10,2}$ with initial data $\omega_{0}$ on the time interval $[0,1]$.

We will prove Theorem 1.13 in Section 9; it will essentially be deduced from Theorem 1.12 by embedding $\mathbb{R}^{2} \times \mathbb{R} / \mathbb{Z}$ into $\mathbb{R}^{3}$ using (modified) cylindrical coordinates. The resulting dynamics resembles that of axisymmetric Euler equations with swirl (particularly when viewed in the coordinates used in [4,69]. For the true Euler equations, the assumption of axial symmetry does not completely reduce matters to an active scalar equation (in contrast to the situation with an assumption of translation symmetry, as used in the proof of Theorem 1.12) due to the non-constant-coefficient nature of the metric $\eta$ in cylindrical coordinates; however, using the freedom to select the vector potential $A$, we can replace $\eta$ locally with a nearby metric which is constant coefficient in cylindrical coordinates on the support of $\omega$, at which point one can adapt the argument used to prove Theorem 1.12. Due to the use of cylindrical coordinates, the solution should now blow up on a circle; see Figure 3.

Remark 1.14 Another potential type of blowup scenario would be a self-similar blowup (here one would need the vector potential operator $A$ to commute with spa- 
tial dilations in an appropriate fashion). In the case of the true Euler equations, the arguments of Chae $[10,12,13]$ preclude non-trivial self-similar solutions in which the vorticity decays rapidly at infinity; however, this leaves open the possibility of nontrivial self-similar solutions that decay only slowly at infinity. However, we were not able to construct such solutions while keeping $A$ self-adjoint and positive semi-definite.

Remark 1.15 There are numerous issues preventing one from adapting these generalised Euler equation blowup results to the case of the generalised Navier-Stokes systems (in the spirit of [64]). The most proximate issue is that all of the results rely in one way or another on the conservation of circulation (Proposition 1.6(i)), which is no longer true for Navier-Stokes type equations. However, even if one could obtain bounds on circulation for Navier-Stokes that were of the same order as what one obtains for Euler, the blowup results in Theorem 1.12 and Theorem 1.13 would still create solutions that presumably blow up on a one-dimensional set, which cannot occur for Navier-Stokes equations by the well known results of Caffarelli, Kohn, and Nirenberg [7]. The blowup result in Theorem 1.11, which concentrates only at a point, avoids this problem; however, the scaling is still (barely) unfavorable due to the assumption of bounded circulation (which turns out to be a stronger condition, from the perspective of scaling analysis, than conservation of energy). The numerology is as follows. At a time $t$ close to the blowup time $T_{*}$, one expects the vortex lines to pinch in a disk of radius comparable to $\sqrt{T_{*}-t}$ (see Figure 1). As this disk has area comparable to $T_{*}-t$, conservation (or at least boundedness) of circulation suggests that the vorticity $\omega$ is of size about $\frac{1}{T_{*}-t}$ on this disk, which corresponds on the level of scaling to a velocity comparable to $\frac{1}{\sqrt{T_{*}-t}}$. Thus far the numerology is self-consistent, but in the case of Navier-Stokes, a viscosity term $\Delta u$ in (1.1) would now be expected to be comparable to $\frac{1}{\left(T_{*}-t\right)^{3 / 2}}$, which is also the order of the transport term $(u \cdot \nabla) u$. Thus we expect the viscosity effects to be comparable to the nonlinear effects, creating a "critical" scenario (analogous to two-dimensional Navier-Stokes) which leads one to predict that the blowup scenario will not occur. It may be possible to still obtain blowup by weakening the viscosity term to something like $\Delta^{\alpha} u$ for some $0<\alpha<1$, but with the full viscosity term $\Delta u$, it appears that this blowup scenario can only occur if either the viscosity somehow causes a significant increase in circulation, or if there is a lot of "looping" of the vortex lines that causes the circulation through a small disk to become very large due to the vortex lines passing through the disk multiple times.

Remark 1.16 The blowup mechanisms in this paper behave a little differently from the locally (approximately) discretely self-similar solutions proposed in [6] (which is in turn modeled on the dynamics seen in [64]). We have already discussed the numerology of the blowup in Theorem 1.11 in the previous remark; now we discuss the numerology in Theorem 1.12 (the situation for Theorem 1.13 is similar). At time $t$ close to the final blowup time $T_{*}$, one expects a particularly strong amount of vorticity (with $\omega$ comparable to $\frac{1}{T_{*}-t}$ ) in a narrow tube of radius about $T_{*}-t$ and sidelength 1 (the tube will be a neighbourhood of a copy of $\mathbb{R} / \mathbb{Z}$ in $\mathbb{R}^{2} \times \mathbb{R} / \mathbb{Z}$ ); see Figure 2 . This vortex tube will only capture a small fraction (about $T_{*}-t$ ) of the original circulation; the remainder will come from a "wake" of larger vortex tubes trailing this narrow tube. The velocity field $u$ will be comparable to 1 throughout, and will pinch 
the narrowest vortex tube to a line (or more precisely, a copy of $\mathbb{R} / \mathbb{Z}$ ) by time $T_{*}$. This scenario has some resemblance to that in [6], which also involves increasingly narrow vortex tubes that carry less and less circulation, but which have increasingly large pointwise vorticity; however, the tubes in [6] are not completely linear but have some curvature (and their length goes to zero as $t$ approaches $T_{*}$ ); furthermore there is a complicated dynamic in [6] in which pairs of vortex tubes attract and deform to become vortex planes, which then destabilise back into thinner vortex tubes, which is probably not present in the blowup constructed in Theorem 1.12 (or Theorem 1.13). Also, the scaling exponents in [6] are more flexible than the ones here, for instance the width of the vortex tubes in [6] is not constrained to decay linearly in $T_{*}-t$, nor is the vorticity constrained to behave inversely like $\frac{1}{T_{*}-t}$. We do not know how to implement the blowup scenario proposed in [6] using a generalised Euler equation, even if one drops the requirements of self-similarity and positive definiteness for the vector potential operator.

Remark 1.17 The blowup scenarios here are not of the "tube collapse" form ruled out in [29], in which the volume of a vortex tube locally collapses to zero; instead, only a small portion of the volume is either pinched in a neck, or creased on a line or circle. As the vorticity is expected to blow up like $1 /\left(T_{*}-t\right)$, the Beale-Kato-Majda blowup criterion is satisfied (as it must be). The blowup scenarios also do not appear to be compatible with the scenarios ruled out in [21], either because the velocity is unbounded or because the vorticity direction is changing too rapidly. One could presumably use these blowup scenarios as test cases for any future blowup criterion results for the true or generalised Euler equations in a similar fashion.

Remark 1.18 It is also tempting to construct blowup solutions by first choosing the fields $u, \omega$ blowing up in a specified fashion (with $\omega$ being transported by $u$ ) and then designing a vector potential operator $A$ to solve the generalised Euler equations with these choices of fields, in the spirit of $[66,67]$. This seems achievable if one does not require $A$ to be self-adjoint. If however self-adjointness is imposed, then this creates a nonlocal-in-time constraint on the fields $u, \omega$ which makes this approach difficult. Namely, if one writes $\omega=d v$ for some $v \in \Lambda_{1}(\mathcal{M})$, then an integration by parts using the self-adjointness of $A$ reveals that we must have the constraint

$$
\int_{\mathcal{M}}\left\langle v(t), u\left(t^{\prime}\right)\right\rangle d \mathrm{vol}=\int_{\mathcal{M}}\left\langle v\left(t^{\prime}\right), u(t)\right\rangle d \text { vol }
$$

relating the velocity $u$ to the covelocity $v$ for all times $t, t^{\prime}$. We do not know how to design suitably blowing up fields $u, \omega$ obeying this constraint as well as (1.10) other than by solving the generalised Euler equations.

Remark 1.19 We have attempted to design the generalised Euler equations so as to capture as many of the known features of the true Euler equations as possible. However, we should mention ${ }^{9}$ two properties of the Euler equations which are not obeyed by the generalised Euler equations, namely translation invariance and rotation invariance; the

\footnotetext{
9 We thank an anonymous referee for stressing this point.
} 
operators $A$ we construct are inhomogeneous and non-isotropic (indeed, if one were to insist on these properties as well as dilation invariance, one would essentially be restricting the class of generalised Euler equations back to the true Euler equations up to some normalising constants, as the Biot-Savart law is basically determined by these symmetries). In particular, we do not have conservation of momentum (impulse) or angular momentum (moment of impulse) for these equations. On the other hand, the class of generalised Euler equations remains invariant with respect to such symmetries, in particular most of the useful estimates on solutions to the Euler or generalised Euler equations involve function space norms which are invariant with respect to translations or rotations. Also, the conservation laws of impulse and moment of impulse are very rarely used in the local or global regularity theory for the Euler equations, so their loss does not significantly reduce the body of results that should transfer over to the generalised Euler equation setting.

\section{Formal Proof of Conservation Laws}

We now prove Proposition 1.6. In this section all calculations will be formal, in that we do not check that all fields involved are smooth enough and exhibit sufficient decay at infinity to justify invocations of identities such as Stokes' theorem; we also assume here that all closed forms are exact.

Let $\omega, u$ solve the generalised Euler equations with some vector potential operator $A$. For future reference we observe from the divergence-free nature of the velocity field $u$ (or equivalently, that $\mathcal{L}_{u} d$ vol $=0$ ) we (formally) have ${ }^{10}$

$$
\int_{\mathcal{M}} \mathcal{L}_{u} f d \mathrm{vol}=0
$$

for any $f \in \Lambda_{0}(\mathcal{M})$. Applying this with $f=\langle\omega, \alpha\rangle$ for any $\omega \in \Lambda_{k}(\mathcal{M})$ and $\alpha \in \Gamma^{k}(\mathcal{M})$ using the Leibniz rule

$$
\mathcal{L}_{u}\langle\omega, \alpha\rangle=\left\langle\mathcal{L}_{u} \omega, \alpha\right\rangle+\left\langle\omega, \mathcal{L}_{u} \alpha\right\rangle
$$

we (formally) conclude the integration by parts formula

$$
\int_{\mathcal{M}}\left\langle\mathcal{L}_{u} \omega, \alpha\right\rangle d \mathrm{vol}=-\int_{\mathcal{M}}\left\langle\omega, \mathcal{L}_{u} \alpha\right\rangle d \mathrm{vol} .
$$

The proof of the Kelvin circulation theorem (i) is standard. For each time $t$, let $\Phi(t): \mathcal{M} \rightarrow \mathcal{M}$ be the diffeomorphism formed by flowing along the vector field $u$, thus $\Phi(0)$ is the identity and

$$
\partial_{t} \Phi(t, x)=u(t, \Phi(t, x))
$$

$\overline{10}$ See e.g. [2, §3.4] for a definition of the Lie derivative on $k$-vector fields, as well as a proof of the Leibniz rule (2.2). 
for all $t \in \mathbb{R}$ and $x \in \mathcal{M}$. Using the interpretation of a Lie derivative as an infinitesimal diffeomorphism, we have

$$
\partial_{t}\left(\Phi(t)^{*} \alpha\right)=\Phi(t)^{*}\left(\partial_{t} \alpha(t)\right)+\Phi^{*}(t)\left(\mathcal{L}_{u(t)} \alpha(t)\right)
$$

for any time-dependent form or vector field $\alpha$, where $\Phi(t)^{*}$ denotes the pullback by $\Phi(t)$. From (1.10) we thus see that $\Phi(t)^{*} \omega(t)$ is conserved in time, thus giving the Cauchy vorticity formula

$$
\omega(t)=\Phi(t)_{*} \omega(0)
$$

where $\Phi(t)_{*}$ is the pushforward by $\Phi(t)$ (the inverse of $\left.\Phi(t)^{*}\right)$. The Kelvin circulation theorem (i) then follows from the change of variables formula.

From (1.11) and (1.3), $u$ is divergence-free, thus by (1.6)

$$
\mathcal{L}_{u} d \text { vol }=0
$$

and thus by (2.4) $\Phi(t)^{*} d$ vol is conserved in time, thus $\Phi(t)$ is volume-preserving:

$$
\Phi(t)^{*} d \text { vol }=d \text { vol }
$$

Since the Hodge duality operator $*$ from Remark 1.1 is defined using the volume form vol, we conclude that $*$ commutes with $\Phi(t)^{*}$. In particular, we see from the Cauchy vorticity formula $(2.5)$ that

$$
* \omega(t)=\Phi(t)_{*}(* \omega(0)) .
$$

In three dimensions, this gives the transport (ii) of the vortex stream lines.

Now we establish (iii). Let $v(t)$ be a time-dependent 1 -form with $d v=\omega$, then from the product rule and differentiation under the integral sign we have

$$
\partial_{t} H(t)=\int_{\mathbb{R}^{3}} \partial_{t} v \wedge \omega+v \wedge \partial_{t} \omega
$$

Writing $\omega=d v$ and using the Leibniz rule for the exterior derivative, we have

$$
d\left(v \wedge \partial_{t} v\right)=\omega \wedge \partial_{t} v-v \wedge \partial_{t} \omega
$$

As the wedge product is commutative between 1-forms and 2-forms, we have $\omega \wedge \partial_{t} v=$ $\partial_{t} v \wedge \omega$. We conclude upon integrating and using Stokes' theorem that

$$
\partial_{t} H(t)=2 \int_{\mathbb{R}^{3}} v \wedge \partial_{t} \omega
$$

and hence by (1.10)

$$
\partial_{t} H(t)=-2 \int_{\mathbb{R}^{3}} v \wedge \mathcal{L}_{u} d v .
$$


Recall (see e.g. $[2, \S 2.25,3.4]$ ) that the Lie derivative $\mathcal{L}_{u}$ and the exterior derivative $d$ obey the Leibniz rules

$$
\mathcal{L}_{u}(\omega \wedge \lambda)=\left(\mathcal{L}_{u} \omega\right) \wedge \lambda+\omega \wedge\left(\mathcal{L}_{u} \lambda\right)
$$

and

$$
d(\omega \wedge \lambda)=(d \omega) \wedge \lambda+(-1)^{k} \omega \wedge(d \lambda)
$$

for all $\omega \in \Lambda_{k}(\mathcal{M})$ and $\lambda \in \Lambda_{l}(\mathcal{M})$. From these Leibniz rules and (1.7) we have

$$
\mathcal{L}_{u}(v \wedge d v)+d\left(\mathcal{L}_{u} v \wedge v\right)=2 v \wedge \mathcal{L}_{u} d v
$$

and the claim (iii) now follows from (2.7), (2.1), and Stokes' theorem.

Now we prove (iv). From the Leibniz rule and the self-adjointness of $A$, we have

$$
\partial_{t} E(t)=\int_{\mathcal{M}}\left\langle\partial_{t} \omega, A \omega\right\rangle d \mathrm{vol}
$$

Using (1.10), (2.6) and (2.3), we conclude

$$
\partial_{t} E(t)=\int_{\mathcal{M}}\left\langle\omega, \mathcal{L}_{u} A \omega\right\rangle d \mathrm{vol} .
$$

As $\omega$ is closed, and we are working formally, we may write $\omega=d v$ for some 1 -form $v$. By duality, we thus have

$$
\partial_{t} E(t)=\int_{\mathcal{M}}\left\langle v, \delta \mathcal{L}_{u} A \omega\right\rangle d \text { vol } .
$$

Since $\mathcal{L}_{u}$ annihilates $d$ vol, it commutes with $*$; by (1.7) and (1.9) it therefore commutes with $\delta$, thus by (1.11)

$$
\delta \mathcal{L}_{u} A \omega=\mathcal{L}_{u} u=[u, u]=0
$$

(where $[X, Y]$ denotes the Lie bracket of two vector fields $X, Y$ ) and the claim follows.

Finally, we establish (v). From (1.10), we have

$$
\partial_{t} \int_{\mathcal{M}}\langle\omega, \alpha\rangle d \mathrm{vol}=-\int_{\mathcal{M}}\left\langle\mathcal{L}_{u} \omega, \alpha\right\rangle d \mathrm{vol} .
$$

By (2.6) and (2.3), the right-hand side is equal to

$$
\int_{\mathcal{M}}\left\langle\omega, \mathcal{L}_{u} \alpha\right\rangle d \text { vol }
$$

Writing $\omega=d v$ as before, and using (2.3), (1.7) and $d \alpha=X$, we can write this as

$$
-\int_{\mathcal{M}}\left\langle v, \mathcal{L}_{u} X\right\rangle d \text { vol } .
$$


Since

$$
\mathcal{L}_{u} X=[u, X]=-[X, u]=-\mathcal{L}_{X} u
$$

we can use (1.11) to write the previous expression as

$$
\int_{\mathcal{M}}\left\langle v, \mathcal{L}_{X} \delta A \omega\right\rangle d \text { vol }
$$

As $X$ is divergence-free, $\mathcal{L}_{X}$ commutes with $*$ and thus with $\delta$. By duality and $d v=\omega$, the above expression becomes

$$
\int_{\mathcal{M}}\left\langle\omega, \mathcal{L}_{X} A \omega\right\rangle d \text { vol }
$$

Using (2.3) and using the self-adjointness of $A$, this is equal to

$$
-\int_{\mathcal{M}}\left\langle A \mathcal{L}_{X} \omega, \omega\right\rangle d \mathrm{vol}
$$

But as $A$ and $\mathcal{L}_{X}$ commute, the previous two expressions are also negations of each other, and must thus be zero. The claim follows.

\section{Formal Lagrangian Formulation}

As in the preceding section, our calculations here will be purely formal, without regard to issues of smoothness or decay.

Given a divergence-free velocity field $u:[0, T] \rightarrow B^{1}(\mathcal{M})$, we can form the family of volume-preserving diffeomorphisms $\Phi(t): \mathcal{M} \rightarrow \mathcal{M}$ for $t \in[0, T]$ by solving the ODE

$$
\partial_{t} \Phi(t, x)=u(t, \Phi(t, x))
$$

with initial data $\Phi(0, x)=x$. We can then deform this family to a two-parameter family $\Phi(s, t): \mathcal{M} \rightarrow \mathcal{M}$ of volume-preserving diffeomorphisms with $s$ near zero by solving a further ODE

$$
\partial_{s} \Phi(s, t, x)=v(t, \Phi(s, t, x))
$$

for some additional divergence-free velocity field $v:[0, T] \rightarrow B^{1}(\mathcal{M})$, with initial data $\Phi(0, t, x)=\Phi(t, x)$. The velocity field $u$ then deforms in $s$ via the formula

$$
\partial_{t} \Phi(t, x)=u(s, t, \Phi(t, x)) .
$$

Differentiating (3.1) in $t$ and (3.2) in $s$ and comparing at $s=0$ gives the identity

$$
\partial_{s} u+(v \cdot \nabla) u=\partial_{t} v+(u \cdot \nabla) v
$$


since

$$
\mathcal{L}_{u} v=[u, v]=(u \cdot \nabla) v-(v \cdot \nabla) u
$$

we thus have ${ }^{11}$

$$
\partial_{s} u=\partial_{t} v+\mathcal{L}_{u} v .
$$

Let $A: B_{2}(\mathcal{M}) \rightarrow \Gamma^{2}(\mathcal{M})$ be formally self-adjoint. We now introduce the formal Lagrangian

$$
\mathcal{L}[\Phi]:=\frac{1}{2} \int_{0}^{T} \int_{\mathcal{M}}\langle\omega, A \omega\rangle d \operatorname{vol} d t,
$$

where $\omega \in B_{2}(\mathcal{M})$ is closed and solves (1.11); we assume that $\delta A$ is invertible, so that $\omega$ is uniquely determined by $u$. We claim that if $u$ also solves (1.10), then it is a (formal) critical point of the Lagrangian if one holds the endpoints $\Phi(0), \Phi(T)$ fixed; in other words, if one deforms $u$ and $\Phi$ as above using a divergence-free field $v$ that vanishes at the endpoints $t=0, T$, then

$$
\partial_{s} \mathcal{L}[\Phi]=0
$$

at $s=0$. Indeed, from (3.4) and the self-adjointness of $A$, the left-hand side is

$$
\int_{0}^{T} \int_{\mathcal{M}}\left\langle\omega, \partial_{S} A \omega\right\rangle d \operatorname{vol} d t .
$$

As $\omega$ is closed, we can write $\omega=d \alpha$ for some 1-form $\alpha$. Integrating by parts and using (1.11), this quantity can be rewritten as

$$
\int_{0}^{T} \int_{\mathcal{M}}\left\langle\alpha, \partial_{s} u\right\rangle d \operatorname{vol} d t
$$

which by (3.3) and (2.3) is equal to

$$
\int_{0}^{T} \int_{\mathcal{M}}\left\langle-\partial_{t} \alpha-\mathcal{L}_{u} \alpha, v\right\rangle d \operatorname{vol} d t .
$$

As $v$ is divergence-free, we can (formally) write $v=\delta \beta$ for some 2-vector field $\beta$. Integrating by parts using $d \alpha=\omega$, we can rewrite the preceding expression as

$$
\int_{0}^{T} \int_{\mathcal{M}}\left\langle-\partial_{t} \omega-\mathcal{L}_{u} \omega, \beta\right\rangle d \operatorname{vol} d t .
$$

But this vanishes by (1.10).

11 Another way of interpreting the identity (3.3) is as follows. The diffeomorphisms $\Phi$ can be viewed as a trivialisation of the $\mathcal{M}$-bundle over the parameter space $\mathbb{R}^{2}$ of the variables $(s, t)$. The flat connection associated to this trivialisation, when written in terms of the standard trivialisation, correspond to the differential operators $\partial_{t}+\mathcal{L}_{u}$ and $\partial_{s}+\mathcal{L}_{v}$. As the connection is flat, these operators commute, giving (3.3). 
Remark 3.1 Suppose the operator $A$ is (formally) positive definite. Then one can interpret the above calculation as asserting that the generalised Euler equations in Definition 1.2 are the formal geodesic flow on the infinite-dimensional manifold $\operatorname{Sdiff}(\mathcal{M})$ of volume-preserving diffeomorphisms on $\mathcal{M}$, where we endow this manifold with a right-invariant metric whose quadratic form on the tangent space of the identity (which one can identify with divergence-free vector fields $u$ ) is given by $u \mapsto \int_{\mathcal{M}}\langle\omega, A \omega\rangle d$ vol, where $\omega$ obeys (1.11). In the case of the true Euler equations, this fact was famously observed by Arnold [1], as a special case of the Euler-Poincaré formalism, and a rigorous version of these computations was used in [37] to obtain a local existence theorem for the true Euler equations that is close to that in Theorem 1.9. See [71] for some analogous results for the SQG equation. If one drops the requirement that the diffeomorphisms be volume preserving, then there are several compressible fluid equations that also have a rigorous geodesic flow interpretation; see e.g. $[19,38,47,54,73]$. It is thus likely that the formal computations in this section can similarly be made rigorous given suitable hypotheses on the vector potential operator $A$ and on the initial data, but we will not attempt to do so here.

Given the above Lagrangian formulation of the generalised Euler equations in Definition 1.2, it should come as no surprise that the conservation laws in Proposition 1.6 are associated to symmetries of the Lagrangian (3.4), in accordance with Noether's theorem. Indeed, the Kelvin circulation theorem (and hence helicity conservation and stream line conservation) come from the invariance of (3.4) with respect to the right action of $\operatorname{Sdiff}(\mathcal{M})$, while conservation of the Hamiltonian comes (as usual) from time translation symmetry, and conservation of impulse comes from the symmetry arising from the diffeomorphisms $e^{s X}$ generated by the vector field $X$. See [61,65] for further discussion.

\section{Classical Local Existence}

In this section we leave the realm of formal calculations, and prove Theorem 1.9 rigorously. We will rely primarily on the energy method, with some modification at low frequencies to deal with the failure of the generalised Biot-Savart operator $\delta A$ to map $H^{s}$ to $L^{\infty}$ in the $m=2$ case. To abbreviate the notation, we write \|\|$_{\dot{H}^{s}}$ for \|\|$_{\dot{H}^{s}(\mathcal{M})}$, and similarly for \|\|$_{L^{p}},\|\|_{H^{s}}$, etc.. It will also be convenient to use the norm

$$
\|f\|_{L^{p} \cap H^{s}}:=\|f\|_{L^{p}}+\|f\|_{H^{s}} .
$$

Let $\mathcal{M}, d, A, M, p$ be as in Theorem 1.9. For brevity, we drop the dependence of constants on $d, M, A, p$ from the asymptotic notation.

We can of course write the system (1.10), (1.11) as a single equation

$$
\partial_{t} \omega+\mathcal{L}_{\delta A \omega} \omega=0 .
$$

In coordinates, the equation (4.1) becomes

$$
\partial_{t} \omega+(\delta A \omega \cdot \nabla) \omega=\mathcal{O}\left(\left(\nabla^{2} A \omega\right) \omega\right)
$$


where we use $\mathcal{O}(X)$ to denote an expression that has the schematic form of $X$ in the sense that it is a linear combination (with constant coefficients) of components of a tensor of the form $X$ (interpreting all products in $X$ as tensor products).

We first establish uniqueness. Given two solutions $\omega_{1}, \omega_{2} \in X^{s, p}$ to (4.2) with initial data $\omega_{0}$, the difference $\alpha:=\omega_{1}-\omega_{2}$ lies in $X^{s, p}$ and obeys an equation of the form

$$
\partial_{t} \alpha+\left(\delta A \omega_{2} \cdot \nabla\right) \alpha+(\delta A \alpha \cdot \nabla) \omega_{1}=\mathcal{O}\left(\left(\nabla^{2} A \alpha\right) \omega_{1}\right)+\mathcal{O}\left(\left(\nabla^{2} A \omega_{2}\right) \alpha\right)
$$

Taking inner products with $\alpha|\alpha|^{p-2}$ and integrating using the divergence-free nature of $\delta A \omega_{2}$, we obtain ${ }^{12}$ the inequality

$$
\partial_{t}\|\alpha\|_{L^{p}} \lesssim\left\|(\delta A \alpha \cdot \nabla) \omega_{1}\right\|_{L^{p}}+\left\|\left(\nabla^{2} A \alpha\right) \omega_{1}\right\|_{L^{p}}+\left\|\left(\nabla^{2} A \omega_{2}\right) \alpha\right\|_{L^{p}}
$$

and hence by Hölder's inequality one has

$$
\begin{aligned}
\partial_{t}\|\alpha\|_{L^{p}} \lesssim & \left\|P_{\mathrm{lo}} \nabla A \alpha\right\|_{L^{q}}\left\|\nabla \omega_{1}\right\|_{L^{m}}+\left\|P_{\mathrm{hi}} \nabla A \alpha\right\|_{L^{r}}\left\|\nabla \omega_{1}\right\|_{L^{d}} \\
& +\left\|\nabla^{2} A \alpha\right\|_{L^{p}}\left\|\omega_{1}\right\|_{L^{\infty}}+\left\|\nabla^{2} A \omega_{2}\right\|_{L^{\infty}}\|\alpha\|_{L^{p}}
\end{aligned}
$$

where $\frac{1}{q}:=\frac{1}{p}-\frac{1}{m}, \frac{1}{r}:=\frac{1}{p}-\frac{1}{d}$, and $P_{\mathrm{lo}}, P_{\mathrm{hi}}$ are the Fourier projections to frequencies $|\xi| \leqslant 1$ and $|\xi|>1$ respectively. From Sobolev embedding in $\mathbb{R}^{m} \times(\mathbb{R} / \mathbb{Z})^{d-m},(1.20)$, and the hypothesis $s>\frac{d}{2}+1$, one has

$$
\begin{aligned}
\left\|P_{\mathrm{lo}} \nabla A \alpha\right\|_{L^{q}},\left\|P_{\mathrm{hi}} \nabla A \alpha\right\|_{L^{r}} & \lesssim\left\|\nabla^{2} A \alpha\right\|_{L^{p}} \\
\left\|\nabla \omega_{1}\right\|_{L^{m}},\left\|\nabla \omega_{1}\right\|_{L^{d}},\left\|\omega_{1}\right\|_{L^{\infty}} & \lesssim\left\|\omega_{1}\right\|_{H^{s}} \lesssim\left\|\omega_{1}\right\|_{X^{s, p}} \\
\left\|\nabla^{2} A \omega_{2}\right\|_{L^{\infty}} & \lesssim\left\|\nabla^{2} A \omega_{2}\right\|_{H^{s}} \lesssim\left\|\omega_{2}\right\|_{H^{s}} \lesssim\left\|\omega_{2}\right\|_{X^{s, p}} .
\end{aligned}
$$

Also, from Definition 1.7, the operator $\nabla^{2} A$ is bounded on $L^{2}$ and has a kernel obeying Calderón-Zygmund estimates, so is bounded on $L^{p}$ by Calderón-Zygmund theory (see e.g. [62]). We conclude that

$$
\partial_{t}\|\alpha\|_{L^{p}} \lesssim\left(\left\|\omega_{1}\right\|_{X^{s, p}}+\left\|\omega_{2}\right\|_{X^{s, p}}\right)\|\alpha\|_{L^{p}}
$$

Since $\alpha(0)=0$, we conclude from Gronwall's inequality that $\alpha=0$ identically, giving uniqueness.

Next, we show existence of (weak) solutions using a standard viscosity method which we briefly sketch here; later on we will upgrade the regularity of solutions from weak to strong. For any $\varepsilon>0$ we can consider the generalised Navier-Stokes equation

$$
\partial_{t} \omega+\mathcal{L}_{\delta A \omega} \omega+\varepsilon \Delta \omega=0
$$

12 To be more rigorous here, one could obtain instead a transport equation for $\left(\varepsilon^{2}+|\alpha|^{2}\right)^{p / 2}$ for $\varepsilon>0$, run the Gronwall argument below for the quantity $\left\|\left(\varepsilon^{2}+|\alpha|^{2}\right)^{1 / 2}\right\|_{L^{p}}$, and then send $\varepsilon$ to zero; we leave the details to the interested reader. Similarly for other arguments in this section involving derivatives of $L^{p}$ or $L^{2}$ norms. 
(recall in this paper that $\Delta$ denotes the Hodge Laplacian, which is positive semidefinite). We can write this equation schematically as

$$
\partial_{t} \omega+\varepsilon \Delta \omega=\mathcal{O}(\nabla A \omega \nabla \omega)+\mathcal{O}\left(\left(\nabla^{2} A \omega\right) \omega\right)
$$

From repeated application of the Hölder and Sobolev inequalities, as well as (1.20), the $L^{p}$ boundedness of $\nabla^{2} A$, and the hypothesis $s>\frac{d}{2}+1$, one can check that if $\omega_{1}, \omega_{2} \in L^{p} \cap H^{s+1} \cap B_{2}(\mathcal{M})$, then the expression $\mathcal{O}\left(\nabla A \omega_{1} \nabla \omega_{2}\right)+\mathcal{O}\left(\left(\nabla^{2} A \omega_{1}\right) \omega_{2}\right)$ lies in $L^{p} \cap H^{s}(\mathcal{M})$, and that this operation is locally Lipschitz ${ }^{13}$ in the $\omega_{1}$ and $\omega_{2}$ variables in the indicated norms. By running a contraction mapping argument that places $\omega$ in the function space

$$
C^{0}\left([0, T] \rightarrow L^{p} \cap H^{s} \cap B_{2}(\mathcal{M})\right) \cap L^{2}\left([0, T] \rightarrow H^{s+1} \cap B_{2}(\mathcal{M})\right),
$$

and using the parabolic smoothing effects of the heat equation (and also noting that the Lie derivative operator $\mathcal{L}_{\delta A \omega}$ and the Hodge Laplacian $\Delta$ both preserve the space $B_{2}(\mathcal{M})$ of closed 2-forms), one can then construct local solutions in (4.4) in the above space that can be continued as long as one has a uniform bound on the quantity

$$
\|\omega(t)\|_{L^{p} \cap H^{s}} .
$$

But for any constant coefficient differential operator $D$ of order $k$ for some $0 \leqslant k \leqslant s$ (with no lower order terms), we have upon differentiating (4.4) by $D$ that

$$
\partial_{t} D \omega+(\delta A \omega \cdot \nabla) D \omega+\varepsilon \Delta D \omega=F
$$

where

$$
F:=[\delta A \omega, D] \cdot \nabla \omega+\mathcal{O}\left(D\left(\left(\nabla^{2} A \omega\right) \omega\right)\right)
$$

and $[A, B]:=A B-B A$. Multiplying by $D \omega$ and integrating by parts, we obtain an energy inequality of the form

$$
\partial_{t}\|D \omega\|_{L^{2}}^{2} \lesssim\|F\|_{L^{2}}\|D \omega\|_{L^{2}}
$$

where the implied constant does not depend on $\varepsilon$. However, using the Moser estimate

$$
\|D(u v)\|_{L^{2}} \lesssim\|u\|_{\dot{H}^{k}}\|v\|_{L^{\infty}}+\|u\|_{L^{\infty}}\|v\|_{\dot{H}^{k}}
$$

\footnotetext{
13 Indeed, $\nabla A \omega_{1}, \nabla^{2} A \omega_{1}, \nabla \omega_{2}, \omega_{2}$ both lie in $H^{s}(\mathcal{M})$ thanks to (1.20), and the product of two functions in $H^{s}(\mathcal{M})$ lies in both $H^{s}(\mathcal{M})$ and $L^{p}(\mathcal{M})$ by the Leibniz rule and the Hölder and Sobolev inequalities.
} 
(see e.g. $[68,(2.0 .22)])$, as well as the commutator estimate ${ }^{14}$

$$
\|[u, D] \nabla v\|_{L^{2}} \lesssim\|u\|_{\dot{H}^{k+1}}\|v\|_{L^{\infty}}+\|\nabla u\|_{L^{\infty}}\|v\|_{\dot{H}^{k}}
$$

(see e.g. [52,53], noting the claim is trivial for $k=0$ ), we see that

$$
\|F\|_{L^{2}} \lesssim\left\|\nabla^{2} A \omega\right\|_{L^{\infty}}\left\|\nabla^{k} \omega\right\|_{L^{2}}+\left\|\nabla^{k+2} A \omega\right\|_{L^{2}}\|\omega\|_{L^{\infty}}
$$

Applying Sobolev embedding then gives

$$
\|F\|_{L^{2}} \lesssim\left\|\nabla^{2} A \omega\right\|_{H^{s}}\|\omega\|_{H^{s}} .
$$

Hence by (1.20) and summing over a suitable choice of $D$ we have

$$
\partial_{t}\|\omega\|_{H^{s}}^{2} \lesssim\|\omega\|_{H^{s}}^{3}
$$

Since $\|\omega(0)\|_{H^{s}} \lesssim M$, this gives an a priori bound

$$
\|\omega(t)\|_{H^{s}} \lesssim M
$$

for $0 \leqslant t \leqslant T$, if $T$ is sufficiently small depending on the bound $M$.

Now we need to control the $L^{p}$ component in (4.6). Since $\nabla^{2} A$ is bounded in $L^{p}, \nabla^{2} A \omega$ has a $L^{p}$ norm of $O\left(\|\omega\|_{L^{p}}\right)$. In particular, from Sobolev embedding and Hölder we have

$$
\begin{aligned}
\left\|\mathcal{O}(\nabla A \omega \nabla \omega)+\mathcal{O}\left(\left(\nabla^{2} A \omega\right) \omega\right)\right\|_{L^{p}} & \lesssim\|\nabla A \omega\|_{L^{q}}\|\nabla \omega\|_{L^{2}}+\left\|\nabla^{2} A \omega\right\|_{L^{\infty}}\|\omega\|_{L^{p}} \\
& \lesssim\left\|\nabla^{2} A \omega\right\|_{L^{p} \cap H^{s}}\|\omega\|_{H^{s}}+\left\|\nabla^{2} A \omega\right\|_{H^{s}}\|\omega\|_{L^{p}} \\
& \lesssim\|\omega\|_{L^{p} \cap H^{s}}\|\omega\|_{H^{s}}
\end{aligned}
$$

where $1 / q:=1 / p-1 / 2$, and hence by (4.5), (4.12), and the contractivity of the heat semigroup in $L^{p}$

$$
\partial_{t}\|\omega\|_{L^{p}} \lesssim M 1+\|\omega\|_{L^{p}}
$$

and hence by Gronwall's inequality we have

$$
\sup _{0 \leqslant t \leqslant T}\|\omega(t)\|_{L^{p}} \lesssim M, T 1
$$

\footnotetext{
14 The commutator estimate would usually have $\|u\|_{\dot{H}^{k}}\|\nabla v\|_{L^{\infty}}$ in place of $\|u\|_{\dot{H}^{k+1}}\|v\|_{L^{\infty}}$ here, but it is not difficult to adapt the standard (paraproduct-based) proof of the estimate to also establish (4.9) as written. Indeed, one can use the Leibniz rule to write $[u, D] \nabla v$ as $\sum_{i=1}^{k} \mathcal{O}\left(\nabla^{i} u \nabla^{k+1-i} v\right)$; the contribution of the cases $i=1, i=k+1$ are trivial, and all intermediate cases can be handled by paraproducts or Littlewood-Paley decomposition.
} 
giving the required uniform bound on (4.6). This a priori bound allows us to continue the solution to (4.4) up to a time $T>0$ that is independent of $\varepsilon$. A standard compactness argument sending $\varepsilon \rightarrow 0$ (and noting from the Rellich compactness theorem that weak convergence in $L^{p} \cap H^{s}$ implies strong convergence in $C^{1}$ ) then gives a (distributional) solution to the inviscid system (1.10), (1.11) with the regularity

$$
\omega \in L^{\infty}\left([0, T] \rightarrow L^{p} \cap H^{s} \cap B_{2}(\mathcal{M})\right)
$$

This is not quite smooth enough to place $\omega$ in $X^{s, p}$ (mainly because of the lack of continuity in time); we will upgrade the regularity of $\omega$ shortly.

To prove continuous dependence on the initial data, we use an argument originally due to Bona and Smith [5] (see also the survey of Tzvetkov [70]). Let $\omega_{0} \in L^{p} \cap H^{s} \cap$ $B_{2}(\mathcal{M})$ with

$$
\left\|\omega_{0}\right\|_{L^{p} \cap H^{s}}<M
$$

Let $\omega_{0}^{\prime} \in L^{p} \cap H^{s+1} \cap B_{2}(\mathcal{M})$ be a suitable mollification of $\omega_{0}$ which also obeys the bound

$$
\left\|\omega_{0}^{\prime}\right\|_{L^{p} \cap H^{s}}<M
$$

we will choose $\omega_{0}^{\prime}$ more precisely later. Let $\omega \in L^{\infty}\left([0, T] \rightarrow L^{p} \cap H^{s} \cap B_{2}(\mathcal{M})\right)$ be a solution to (4.1) with initial data $\omega_{0}$ constructed by the preceding compactness argument, and similarly define $\omega^{\prime} \in L^{\infty}\left([0, T] \rightarrow L^{p} \cap H^{s} \cap B_{2}(\mathcal{M})\right)$. From (4.12), (4.14) we have the bounds to be the solution to (4.1) with initial data $\omega_{0}^{\prime}$. Then we have

$$
\|\omega(t)\|_{L^{p} \cap H^{s}},\left\|\omega^{\prime}(t)\right\|_{L^{p} \cap H^{s}} \lesssim_{M, T} 1
$$

for all $0 \leqslant t \leqslant T$. A routine modification of the proof of (4.11) yields the a priori bound

$$
\partial_{t}\left\|\omega^{\prime}\right\|_{H^{s+1}}^{2} \lesssim\|\omega\|_{H^{s}}\left\|\omega^{\prime}\right\|_{H^{s+1}}^{2}
$$

which by Gronwall's inequality and (4.16) leads to the bound

$$
\left\|\omega^{\prime}(t)\right\|_{H^{s+1}} \lesssim_{M, T}\left\|\omega_{0}^{\prime}\right\|_{H^{s+1}}
$$

for all $t \in[0, T]$.

Next, we set $\alpha:=\omega^{\prime}-\omega$. As in (4.3), we have the difference equation

$$
\partial_{t} \alpha+(\delta A \omega \cdot \nabla) \alpha+(\delta A \alpha \cdot \nabla) \omega^{\prime}=\mathcal{O}\left(\left(\nabla^{2} A \alpha\right) \omega^{\prime}\right)+\mathcal{O}\left(\left(\nabla^{2} A \omega\right) \alpha\right) .
$$

Taking inner products with $\alpha|\alpha|^{p-2}$ as before, we see that

$$
\partial_{t}\|\alpha\|_{L^{p}} \lesssim\left\|\nabla A \alpha \nabla \omega^{\prime}\right\|_{L^{p}}+\left\|\nabla^{2} A \alpha \omega^{\prime}\right\|_{L^{p}}+\left\|\nabla^{2} A \omega \alpha\right\|_{L^{p}}
$$


Using Sobolev embedding and Hölder as in (4.13), as well as the boundedness of $\nabla^{2} A$ on $L^{p}$, we conclude that

$$
\partial_{t}\|\alpha\|_{L^{p}} \lesssim\|\alpha\|_{L^{p}}\left(\left\|\omega^{\prime}\right\|_{H^{s}}+\|\omega\|_{H^{s}}\right)
$$

and hence by (4.16) and Gronwall's inequality we have

$$
\|\alpha(t)\|_{L^{p}} \lesssim_{M, T}\|\alpha(0)\|_{L^{p}}
$$

for all $t \in[0, T]$.

Next, if $D$ is a constant coefficient operator of order $k$ for some $k \leqslant s$, then upon applying $D$ to (4.18) we have

$$
\partial_{t} D \alpha+(\delta A \omega \cdot \nabla) D \alpha=F^{\prime}
$$

where

$$
\begin{aligned}
F^{\prime}:= & {[\delta A \omega, D] \cdot \nabla \alpha+[\delta A \alpha, D] \cdot \nabla \omega^{\prime}-(\delta A \alpha \cdot \nabla) D \omega^{\prime}+\mathcal{O}\left(D\left(\left(\nabla^{2} A \alpha\right) \omega\right)\right) } \\
& +\mathcal{O}\left(D\left(\left(\nabla^{2} A \omega^{\prime}\right) \alpha\right)\right) .
\end{aligned}
$$

Multiplying (4.20) by $D \alpha$ and then integrating by parts, we conclude that

$$
\partial_{t}\|D \alpha\|_{L^{2}}^{2} \lesssim\|D \alpha\|_{L^{2}}\left\|F^{\prime}\right\|_{L^{2}}
$$

On the other hand, by using (4.8), (4.9), (1.20) as before, followed by (4.16), we have

$$
\begin{aligned}
\left\|F^{\prime}\right\|_{L^{2}} & \lesssim\|\alpha\|_{H^{k}}\left(\|\omega\|_{H^{s}}+\left\|\omega^{\prime}\right\|_{H^{s}}\right)+\left\|(\delta A \alpha \cdot \nabla) D \omega^{\prime}\right\|_{L^{2}} \\
& \lesssim M, T\|\alpha\|_{H^{k}}+\|\nabla A \alpha\|_{L^{\infty}}\left\|\omega^{\prime}\right\|_{H^{k+1}} .
\end{aligned}
$$

Summing over a suitable set of $D$, we conclude that

$$
\partial_{t}\|\alpha\|_{H^{k}}^{2} \lesssim_{M, T}\|\alpha\|_{H^{k}}^{2}+\|\alpha\|_{H^{k}}\|\nabla A \alpha\|_{L^{\infty}}\left\|\omega^{\prime}\right\|_{H^{k+1}}
$$

and thus

$$
\partial_{t}\|\alpha\|_{H^{k}} \lesssim M, T\|\alpha\|_{H^{k}}+\|\nabla A \alpha\|_{L^{\infty}}\left\|\omega^{\prime}\right\|_{H^{k+1}}
$$

for any $0 \leqslant k \leqslant s$.

When $m \geqslant 3$, we can use Sobolev embedding and (1.20) to bound

$$
\|\nabla A \alpha\|_{L^{\infty}} \lesssim\left\|\nabla^{2} A \alpha\right\|_{H^{s-1}} \lesssim\|\alpha\|_{H^{s-1}}
$$

However when $m=2$ the situation is more delicate. If $P_{h i}$ and $P_{l o}$ denote the Fourier projections used previously, we have

$$
\left\|P_{h i} \nabla A \alpha\right\|_{L^{\infty}} \lesssim\left\|\nabla^{2} A \alpha\right\|_{H^{s-1}} \lesssim\|\alpha\|_{H^{s-1}}
$$


For $P_{l o}$, we see for any $N \geqslant 2$ using the Bernstein and Cauchy-Schwarz inequalities, as well as Plancherel's theorem, that

$$
\begin{aligned}
\left\|P_{l o} \nabla A \alpha\right\|_{L^{\infty}} & \lesssim \sum_{M \leqslant 1}\left\|P_{M} \nabla A \alpha\right\|_{L^{\infty}} \\
& \lesssim \sum_{M \leqslant 1}\left\|P_{M} \nabla^{2} A \alpha\right\|_{L^{2}} \\
& \lesssim \sum_{M \leqslant N^{-C}} M^{1 / 2-1 / p}\left\|\nabla^{2} A \alpha\right\|_{L^{p}}+\sum_{N^{-C}<M \leqslant 1}\left\|P_{M} \nabla^{2} A \alpha\right\|_{L^{2}} \\
& \lesssim \frac{1}{N^{2}}\left\|\nabla^{2} A \alpha\right\|_{L^{p}}+\sqrt{\log N}\left(\sum_{N^{-C}<M \leqslant 1}\left\|P_{M} \nabla^{2} A \alpha\right\|_{L^{2}}^{2}\right)^{1 / 2} \\
& \lesssim \frac{1}{N^{2}}\left\|\nabla^{2} A \alpha\right\|_{L^{p}}+\sqrt{\log N}\left\|\nabla^{2} A \alpha\right\|_{L^{2}}
\end{aligned}
$$

where $C:=2 /(1 / p-1 / 2), M$ ranges over dyadic numbers $M=2^{m}, m \in \mathbb{Z}$, and $P_{M}$ is a Littlewood-Paley type Fourier projection to frequencies comparable to $M$. Since $\nabla^{2} A$ is bounded on $L^{p}, L^{2}$, and $H^{s-1}$, we conclude for any choice of $m$ that

$$
\|\nabla A \alpha\|_{L^{\infty}} \lesssim \frac{1}{N^{2}}\|\alpha\|_{L^{p}}+\sqrt{\log N}\|\alpha\|_{L^{2}}+\|\alpha\|_{H^{s-1}}
$$

Inserting these bounds and (4.19) into (4.21), we have

$$
\partial_{t}\|\alpha\|_{H^{k}} \lesssim M, T\|\alpha\|_{H^{k}}+\left(\frac{1}{N^{2}}\|\alpha(0)\|_{L^{p}}+\sqrt{\log N}\|\alpha\|_{L^{2}}+\|\alpha\|_{H^{s-1}}\right)\left\|\omega^{\prime}\right\|_{H^{k+1}}
$$

for any $0 \leqslant k \leqslant s$ and $N \geqslant 2$. We first apply this bound with $k=0$ using (4.16) to obtain

$$
\partial_{t}\|\alpha\|_{L^{2}} \lesssim M, T \frac{1}{N}\|\alpha(0)\|_{L^{p}}+\sqrt{\log N}\|\alpha\|_{L^{2}}
$$

and hence by Gronwall's inequality

$$
\|\alpha(t)\|_{L^{2}} \lesssim M, T \exp \left(O_{M, T}(\sqrt{\log N})\right)\left(\frac{1}{N^{2}}\|\alpha(0)\|_{L^{p}}+\|\alpha(0)\|_{L^{2}}\right)
$$

which on re-insertion back into (4.22) and conceding some powers of $N$ give

$$
\partial_{t}\|\alpha\|_{H^{k}} \lesssim M, T\|\alpha\|_{H^{k}}+\left(\frac{1}{N}\|\alpha(0)\|_{L^{p}}+N^{s-1}\|\alpha(0)\|_{L^{2}}+\|\alpha\|_{H^{s-1}}\right)\left\|\omega^{\prime}\right\|_{H^{k+1}}
$$


Applying this bound with $k=s-1$ and using (4.16), we conclude that

$$
\partial_{t}\|\alpha\|_{H^{s-1}} \lesssim M, T\|\alpha\|_{H^{s-1}}+\frac{1}{N}\|\alpha(0)\|_{L^{p}}+N^{s-1}\|\alpha(0)\|_{L^{2}}
$$

and hence by Gronwall's inequality

$$
\|\alpha(t)\|_{H^{s-1}} \lesssim_{M, T}\|\alpha(0)\|_{H^{s-1}}+\frac{1}{N}\|\alpha(0)\|_{L^{p}}+N^{s-1}\|\alpha(0)\|_{L^{2}}
$$

for any $0 \leqslant t \leqslant T$. Inserting this back into (4.22) for $k=s$ and using (4.17), we have

$$
\partial_{t}\|\alpha\|_{H^{s}} \lesssim M, T\|\alpha\|_{H^{s}}+\left(\frac{1}{N}\|\alpha(0)\|_{L^{p}}+N^{s-1}\|\alpha(0)\|_{L^{2}}+\|\alpha(0)\|_{H^{s-1}}\right)\left\|\omega^{\prime}(0)\right\|_{H^{s+1}}
$$

and hence by Gronwall's inequality

$$
\|\alpha(t)\|_{H^{s}} \lesssim M, T\|\alpha(0)\|_{H^{s}}+\left(\frac{1}{N}\|\alpha(0)\|_{L^{p}}+N^{s-1}\|\alpha(0)\|_{L^{2}}+\|\alpha(0)\|_{H^{s-1}}\right)\left\|\omega^{\prime}(0)\right\|_{H^{s+1}}
$$

for any $0 \leqslant t \leqslant T$. Combining this with (4.19) and the definition of $\alpha$, we have

$$
\begin{aligned}
& \left\|\omega^{\prime}(t)-\omega(t)\right\|_{L^{p} \cap H^{s}} \lesssim M, T\left\|\omega_{0}^{\prime}-\omega_{0}\right\|_{L^{p} \cap H^{s}} \\
& \quad+\left(\frac{1}{N}\left\|\omega_{0}^{\prime}-\omega_{0}\right\|_{L^{p}}+N^{s-1}\left\|\omega_{0}^{\prime}-\omega_{0}\right\|_{L^{2}}+\left\|\omega_{0}^{\prime}-\omega_{0}\right\|_{H^{s-1}}\right)\left\|\omega_{0}^{\prime}\right\|_{H^{s+1}} .
\end{aligned}
$$

Let $\varepsilon>0$. If we let $\tilde{\omega}_{0}$ be initial data in $L^{p} \cap H^{s} \cap B_{2}(\mathcal{M})$ that is sufficiently close to $\omega_{0}$ in $L^{p} \cap H^{s}$ norm (depending on $\varepsilon, N$ ), and let $\tilde{\omega}$ be the corresponding solution to $(4.1)$, the same argument (replacing $\omega$ with $\tilde{\omega}$ ) gives

$$
\begin{aligned}
& \left\|\omega^{\prime}(t)-\tilde{\omega}(t)\right\|_{L^{p} \cap H^{s}} \lesssim M, T\left\|\omega_{0}^{\prime}-\omega_{0}\right\|_{L^{p} \cap H^{s}} \\
& \quad+\left(\frac{1}{N}\left\|\omega_{0}^{\prime}-\omega_{0}\right\|_{L^{p}}+N^{s-1}\left\|\omega_{0}^{\prime}-\omega_{0}\right\|_{L^{2}}+\left\|\omega_{0}^{\prime}-\omega_{0}\right\|_{H^{s-1}}\right)\left\|\omega_{0}^{\prime}\right\|_{H^{s+1}}+\varepsilon
\end{aligned}
$$

and thus by the triangle inequality

$$
\begin{aligned}
& \|\omega(t)-\tilde{\omega}(t)\|_{L^{p} \cap H^{s}} \lesssim M, T\left\|\omega_{0}^{\prime}-\omega_{0}\right\|_{L^{p} \cap H^{s}} \\
& \quad+\left(\frac{1}{N}\left\|\omega_{0}^{\prime}-\omega_{0}\right\|_{L^{p}}+N^{s-1}\left\|\omega_{0}^{\prime}-\omega_{0}\right\|_{L^{2}}+\left\|\omega_{0}^{\prime}-\omega_{0}\right\|_{H^{s-1}}\right)\left\|\omega_{0}^{\prime}\right\|_{H^{s+1}}+\varepsilon
\end{aligned}
$$

for $\tilde{\omega}_{0}$ sufficiently close to $\omega_{0}$ in $L^{p} \cap H^{s} \cap B_{2}(\mathcal{M})$.

If we now let $\omega_{0}^{\prime}$ be a smoothed Fourier projection of $\omega_{0}$ (of Littlewood-Paley type) to frequencies less than $N$, we see from Plancherel's theorem and dominated convergence that

$$
\begin{aligned}
&\left\|\omega_{0}^{\prime}-\omega_{0}\right\|_{L^{p} \cap H^{s}} \lesssim \varepsilon \\
& N^{s}\left\|\omega_{0}^{\prime}-\omega_{0}\right\|_{L^{2}} \lesssim \varepsilon
\end{aligned}
$$




$$
\begin{aligned}
N\left\|\omega_{0}^{\prime}-\omega_{0}\right\|_{H^{s-1}} & \lesssim \varepsilon \\
\frac{1}{N}\left\|\omega_{0}^{\prime}\right\|_{H^{s+1}} & \lesssim \varepsilon
\end{aligned}
$$

for $N$ large enough, and thus

$$
\sup _{0 \leqslant t \leqslant T}\|\omega(t)-\tilde{\omega}(t)\|_{L^{p} \cap H^{s}} \lesssim M, T \varepsilon
$$

whenever $\tilde{\omega}_{0}$ is sufficiently close to $\omega_{0}$. Writing $\beta:=\omega-\tilde{\omega}$, we have the difference equation

$$
\partial_{t} \beta=-(\delta A \tilde{\omega} \cdot \nabla) \beta-(\delta A \beta \cdot \nabla) \omega+\mathcal{O}\left(\left(\nabla^{2} A \beta\right) \omega\right)+\mathcal{O}\left(\left(\nabla^{2} A \tilde{\omega}\right) \beta\right) .
$$

Using (4.25), (4.12), and (1.20), all the terms on the right-hand side can be computed to have an $H^{s-1}$ norm of $O_{M, T}(\varepsilon)$, and so

$$
\sup _{0 \leqslant t \leqslant T}\left\|\partial_{t}(\omega(t)-\tilde{\omega}(t))\right\|_{H^{s-1}} \lesssim M, T \varepsilon
$$

The estimates (4.25), (4.26) will give continuous dependence of the solution map $\omega_{0} \mapsto \omega$ from $L^{p} \cap H^{s} \cap B_{2}(\mathcal{M})$ to $X^{s, p}$ as soon as we establish that the solution $\omega$ actually lies in $X^{s, p}$. We already have the $L^{\infty}$ regularity (4.15); we now upgrade this to $C^{0}$ regularity. By approximating $\omega_{0}$ by initial data in $L^{p} \cap H^{s+1} \cap B_{2}(\mathcal{M})$ and using the continuity estimates already established, it suffices to establish $C^{0}$ regularity under the hypothesis that $\omega_{0} \in L^{p} \cap H^{s+1} \cap B_{2}(\mathcal{M})$. By (4.17) we then have

$$
\|\omega(t)\|_{L^{p}}+\|\omega(t)\|_{H^{s+1}} \lesssim \omega_{0} 1
$$

for all $0 \leqslant t \leqslant T$; from (4.4), the Hölder and Sobolev inequalities, and (1.20) we then have

$$
\left\|\partial_{t} \omega(t)\right\|_{H^{s}} \lesssim \omega_{0} 1
$$

and from repeating the proof of (4.13) we also have

$$
\left\|\partial_{t} \omega(t)\right\|_{L^{p}} \lesssim \omega_{0} 1
$$

and on integrating in $t$ this gives the desired continuity in time in the $L^{p} \cap H^{s}(\mathcal{M})$ topology. Finally, once we know that $\omega$ lies in $C\left([0, T] \rightarrow L^{p} \cap H^{s}(\mathcal{M})\right)$, we can use (4.4), (1.20), and the Hölder and Sobolev inequalities to conclude that $\partial_{t} \omega$ lies in $C\left([0, T] \rightarrow H^{s-1}(\mathcal{M})\right)$, and so $\omega$ lies in $X^{s, p}$ as required. Setting $u:=\delta A \omega$, we also have $u \in Y^{s, p}$ by (1.20) and the boundedness of the Calderón-Zygmund operator $\nabla^{2} A$ on $L^{p}$; this also gives continuous dependence of $u$ on $\omega_{0}$. This concludes the proof of the local wellposedness portion of Theorem 1.9. 
Now we establish the Beale-Kato-Majda criterion. Suppose a priori that we have a solution $\omega \in X^{s, p}, u \in Y^{s, p}$ to (1.10), (1.11) up to some (possibly large) time $0<T_{*}<\infty$ with the bounds

$$
\|\omega(0)\|_{L^{p}}+\|\omega(0)\|_{H^{s}} \leqslant M
$$

and

$$
\int_{0}^{T_{*}}\|\omega(t)\|_{L^{\infty}} d t \leqslant M
$$

for some $0<M<\infty$. Multiplying (4.2) by $\omega$ and integrating, we have

$$
\partial_{t}\|\omega\|_{L^{2}}^{2} \lesssim\left\|\nabla^{2} A \omega\right\|_{L^{2}}\|\omega\|_{L^{\infty}}\|\omega\|_{L^{2}}
$$

and hence by (1.20)

$$
\partial_{t}\|\omega\|_{L^{2}}^{2} \lesssim\|\omega\|_{L^{\infty}}\|\omega\|_{L^{2}}^{2}
$$

and hence by Gronwall's inequality and (4.27), (4.28) one has

$$
\|\omega(t)\|_{L^{2}} \lesssim_{M, T_{*}} 1
$$

for all $0 \leqslant t \leqslant T_{*}$.

Next, let $D$ be a constant coefficient differential operator of order $k \leqslant s$, with no lower order terms. From (4.7), (4.10) we have

$$
\partial_{t}\|D \omega\|_{L^{2}}^{2} \lesssim\left(\left\|\nabla^{2} A \omega\right\|_{L^{\infty}}\left\|\nabla^{k} \omega\right\|_{L^{2}}+\left\|\nabla^{k+2} A \omega\right\|_{L^{2}}\|\omega\|_{L^{\infty}}\right)\|D \omega\|_{L^{2}}
$$

and hence on summing over suitable $D$ and using (1.20)

$$
\partial_{t}\|\omega\|_{H^{s}}^{2} \lesssim\|\omega\|_{H^{s}}^{2}\left(\|\omega\|_{L^{\infty}}+\left\|\nabla^{2} A \omega\right\|_{L^{\infty}}\right)
$$

We now establish a key logarithmic inequality, as in [3]:

Lemma 4.1 We have

$$
\left\|\nabla^{2} A \omega\right\|_{L^{\infty}} \lesssim 1+\|\omega\|_{L^{2}}+\|\omega\|_{L^{\infty}} \log \left(2+\|\omega\|_{H^{s}}\right) .
$$

Proof Let $N \geqslant 2$ be a parameter to be chosen later. From (1.20) and Sobolev embedding we have

$$
\left\|\nabla^{3} A \omega\right\|_{L^{\infty}} \lesssim\left\|\nabla^{2} A \omega\right\|_{H^{s}} \lesssim\|\omega\|_{H^{s}}
$$

and hence for any $x_{0} \in \mathcal{M}$, we have

$$
\left|\nabla^{2} A \omega\left(x_{0}\right)\right| \lesssim N^{d}\left|\int_{\mathcal{M}} \phi\left(N\left(x-x_{0}\right)\right) \nabla^{2} A \omega(x) d \operatorname{vol}(x)\right|+\frac{1}{N}\|\omega\|_{H^{s}}
$$


for some fixed bump function $\phi$ supported on the unit ball $B_{\mathcal{M}}(0,1)$, where of course

$$
B_{\mathcal{M}}\left(x_{0}, r\right):=\left\{x \in \mathcal{M}:\left|x-x_{0}\right| \leqslant r\right\}
$$

is the ball of radius $r$ centred at $x_{0}$ with respect to the distance associated with the Euclidean metric $\eta$ on $\mathcal{M}$. We split $\omega=\omega 1_{B_{\mathcal{M}}\left(x_{0}, 2 / N\right)}+\omega\left(1-1_{B_{\mathcal{M}}\left(x_{0}, 2 / N\right)}\right)$. The former term has an $L^{2}$ norm of $O\left(N^{-d / 2}\|\omega\|_{L^{\infty}}\right)$, hence by (1.20) and Cauchy-Schwarz, we have

$$
N^{d}\left|\int_{\mathcal{M}} \phi\left(N\left(x-x_{0}\right)\right) \nabla^{2} A\left(\omega 1_{B_{\mathcal{M}}\left(x_{0}, 2 / N\right)}\right)(x) d \operatorname{vol}(x)\right| \lesssim\|\omega\|_{L^{\infty}} .
$$

Now we turn to the contribution of $\omega\left(1-1_{B_{\mathcal{M}}\left(x_{0}, 2 / N\right)}\right)$. Using the kernel representation (1.18) of $A$, we can bound

$$
\begin{aligned}
& N^{d} \mid \int_{\mathcal{M}} \phi\left(N\left(x-x_{0}\right)\right) \nabla^{2} A\left(\omega \left(1-1_{\left.\left.B_{\mathcal{M}}\left(x_{0}, 2 / N\right)\right)\right)(x) d \operatorname{vol}(x) \mid} \mid\right.\right. \\
& \quad \lesssim N^{3} \int_{B_{\mathcal{M}}\left(x_{0}, 1 / N\right)} \int_{\mathcal{M} \backslash B_{\mathcal{M}}\left(x_{0}, 2 / N\right)}\left|\nabla_{x}^{2} K(x, y)\right||\omega(y)| d \operatorname{vol}(y) d \operatorname{vol}(x) .
\end{aligned}
$$

From (1.19) one has $\left|\nabla_{x}^{2} K(x, y)\right| \lesssim|x-y|^{-d}+|x-y|^{-m}$. Using $L^{\infty}$ bounds on $\omega$ for $y \in B_{\mathcal{M}}\left(x_{0}, 1\right)$ and $L^{2}$ bounds elsewhere, we can bound the above expression by

$$
\|\omega\|_{L^{\infty}} \log N+\|\omega\|_{L^{2}}
$$

and hence

$$
\left\|\nabla^{2} A \omega\right\|_{L^{\infty}} \lesssim \frac{1}{N}\|\omega\|_{H^{s}}+\|\omega\|_{L^{2}}+\|\omega\|_{L^{\infty}} \log N
$$

Setting $N:=2+\|\omega\|_{H^{s}}$, we obtain the claim.

Using this inequality and (4.29), we thus have

$$
\partial_{t}\|\omega\|_{H^{s}}^{2} \lesssim M, T_{*}\|\omega\|_{H^{s}}^{2}\left(1+\|\omega\|_{L^{\infty}} \log \left(2+\|\omega\|_{H^{s}}\right)\right)
$$

and hence by the chain rule

$$
\partial_{t} \log \left(2+\|\omega\|_{H^{s}}\right) \lesssim_{M, T_{*}}\left(1+\|\omega\|_{L^{\infty}}\right) \log \left(2+\|\omega\|_{H^{s}}\right)
$$

Using Gronwall's inequality and (4.27), we conclude the a priori bound

$$
\|\omega\|_{H^{s}} \lesssim_{M, T_{*}} 1
$$

all the way up to $T_{*}$; repeating the proof of (4.14) we also have

$$
\|\omega\|_{L^{p}} \lesssim M, T_{*} 1
$$


all the way to this time. By the local existence theory already established, this allows one to continue the solution beyond the time $T_{*}$. Taking contrapositives, we obtain the Beale-Kato-Majda blowup criterion.

\section{Non-self-adjoint Blowup: A Simple One-Dimensional Example}

Our three blowup theorems will rely on a "non-self-adjoint blowup" mechanism in which the velocity field $u$ depends on the dynamic field (which will either be a scalar $\theta$ or a 2-form $\omega$, depending on the dimensionality) in a non-self-adjoint fashion (though for the last two of our theorems, we will rely on an embedding trick to make the vector potential operator self-adjoint again). To illustrate this mechanism, we begin with a simple blowup result for a (compressible) one-dimensional equation (a variant of the inviscid Burgers equation). This result will not be directly used elsewhere in the paper, but may help illustrate the basic strategy of the arguments in subsequent sections.

Proposition 5.1 (One-dimensional non-self-adjoint blowup). Let $\theta_{0}: \mathbb{R} \rightarrow \mathbb{R}$ be a smooth function with $\theta_{0}(0)=0$ and $\theta_{0}(x)=1$ for all $x>1 / 4$. Then there does not exist a smooth bounded solution $u, \theta:[0,1] \times \mathbb{R} \rightarrow \mathbb{R}$ to the system

$$
\begin{aligned}
\partial_{t} \theta+u \partial_{x} \theta & =0 \\
u(t, x) & =-\theta(t, 2 x)
\end{aligned}
$$

with initial data $\theta(0, x)=\theta_{0}(x)$.

Note that the negative dilation map that sends a function $x \mapsto \theta(x)$ to the function $x \mapsto-\theta(2 x)$ is non-self-adjoint. The system (5.1), (5.2) transports the field $\theta$ at a position $x$ with a velocity that depends on the value of the field $\theta$ at the position $2 x$; however, due to the non-self-adjointness, the value of $\theta$ at $x$ has no direct impact on the dynamics of $\theta$ at $2 x$. This one-way causality makes it easy to force the $\theta=1$ portion of the solution to collide with the $\theta=0$ portion to create the desired singularity; the point is that the "front" of the $\theta=1$ portion is being driven by the "bulk" of that portion, without any feedback in the opposite direction. This basic dynamic will also power all the rest of the blowup arguments in this paper.

Proof Suppose for contradiction that there are $u, \theta$ with the claimed properties. We use the barrier method, introducing a time-varying barrier $\Omega(t)$ which, on its boundary, expands slower than the velocity field. More precisely, for each time $t \in[0,1]$, let $\Omega(t) \subset \mathbb{R}$ denote the half-line

$$
\Omega(t):=[(1-t) / 2,+\infty)
$$

thus $\Omega(t)$ expands outwards at speed $1 / 2$ until it reaches the origin at time $t=1$; see Figure 4 . Let $T_{*}$ denote the supremum of all the times $0 \leqslant T_{*} \leqslant 1$ such that $\theta(t, x)=1$ for all $0 \leqslant t \leqslant T_{*}$ and $x \in \Omega(t)$. From the initial condition $\theta=\theta_{0}$, and the fact that $\theta$ is transported by the bounded velocity field $u$, we see that $0<T_{*} \leqslant 1$. By continuity we see that $\theta\left(T_{*}\right)$ equals 1 on $\Omega\left(T_{*}\right)$. 


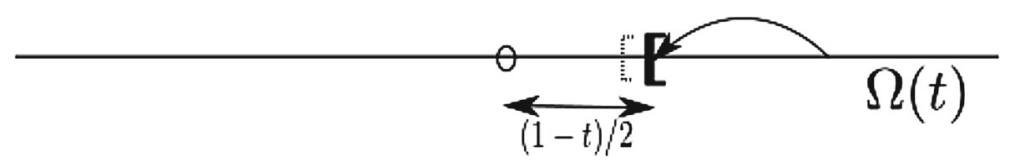

Fig. 4 The region $\Omega(t)$ (to the right of the solid bracket) and a slightly later region $\Omega(t+d t)$ (to the right of the dotted bracket). The active scalar $\theta(t)$ is known to equal one on $\Omega(t)$, and to vanish at the origin (depicted here by a small circle). The curved arrow from $2 x$ to $x$ represents the one-way causality of the (non-self-adjoint) negative dilation operator in (5.2) that sends $x \mapsto \theta(x)$ to $x \mapsto-\theta(2 x)$

From (5.1), (5.2) applied to $x=0$, we have

$$
\partial_{t} \theta(t, 0)=\theta(t, 0) \partial_{x} \theta(t, 0) .
$$

Since $\theta(0,0)=\theta_{0}(0)=0$ and $\theta$ is smooth, we conclude from Gronwall's inequality that $\theta(1,0)=0$. Since $\Omega(1)$ contains 0 , we conclude that $T_{*}$ cannot equal 1 , thus $0<T_{*}<1$.

Let $x_{*}:=\left(1-T_{*}\right) / 2$. By construction of $T_{*}$ and continuity, we have $\theta\left(T_{*}, x_{*}\right)=1$, but $\theta\left(T_{*}, x\right) \neq 1$ for $x$ arbitrarily close to $x_{*}$. On the other hand, we have $\theta(t, x)=1$ for all $0 \leqslant t \leqslant T_{*}$ and $x \in \Omega(t)$. Since $x_{*}$ lies on the boundary of $\Omega\left(T_{*}\right)$, which moves at a velocity of $-1 / 2$, and $\theta$ is transported by the velocity field $u$, we conclude (by the method of characteristics) that

$$
u\left(T_{*}, x_{*}\right) \geqslant-1 / 2,
$$

otherwise one could flow $\theta$ backwards in time from $T_{*}$ and conclude that $\theta(t, x) \neq 1$ for some $t$ slightly less than $T_{*}$ and some $x$ barely inside $\Omega(t)$.

On the other hand, we have $\theta\left(T_{*}, x\right)=1$ for all $x \geq\left(1-T_{*}\right) / 2$, and hence from (5.2) we see that $u\left(T_{*}, x_{*}\right)=-1$. This contradicts (5.3) and gives the claim.

Remark 5.2 The above argument suggests that, at best, the solution $u$ will survive up to time 1 , and for times $t$ close to 1 it will equal 1 on the region $\Omega(t)$ and vanish at and to the left of 0 . However, as the proof of the above proposition is by contradiction, it does not preclude the possibility that the solution $u$ in fact blows up sooner, and possibly with a qualitatively different dynamics ${ }^{15}$ than the one suggested here. Similarly, the arguments used to prove the main theorems in our paper suggest a possible blowup mechanism, but do not ensure that this mechanism actually occurs because the solution may in fact blow up sooner, and in a different fashion, from that mechanism.

\footnotetext{
15 In the case when $\theta$ is non-negative and vanishing to the left of the origin, it may be possible to analyse the solution more carefully using some variant of the method of characteristics to obtain more definitive control on the blowup, for instance it seems possible to show that blowup at a point $x_{0}>0$ cannot occur if the solution remained regular in the region $x \geqslant 2 x_{0}$, which on iteration suggests that blowup can only occur at (or to the left of) the origin. We thank an anonymous referee for this observation.
} 


\section{A Non-self-adjoint Blowup of an SQG-Type Equation}

We now give a two-dimensional version of the argument in the previous section, establishing finite time blow up of an SQG type equation with a non-self-adjoint vector potential operator $A$. The construction here can be viewed as a simplified version of the three-dimensional blowup construction used to establish Theorem 1.11, and will also be embedded directly into the three-dimensional blowup constructions in Theorem 1.12 and Theorem 1.13.

Consider the generalised Euler equation (1.10), (1.11) on $\mathbb{R}^{2}$. We formally write the vector potential operator $A: B_{2}\left(\mathbb{R}^{2}\right) \rightarrow \Gamma^{2}\left(\mathbb{R}^{2}\right)$ in coordinates as

$$
A\left(\theta d x^{1} \wedge d x^{2}\right)=\left(A_{0} \theta\right) \frac{d}{d x^{1}} \wedge \frac{d}{d x^{2}}
$$

for some linear operator $A_{0}: \Lambda_{0}\left(\mathbb{R}^{2}\right) \rightarrow \Lambda_{0}\left(\mathbb{R}^{2}\right)$ and all scalar functions $\theta: \mathbb{R}^{2} \rightarrow \mathbb{R}$. If we write the fields $\omega, u$ in coordinates as

$$
\omega=\theta d x^{1} \wedge d x^{2}
$$

and

$$
u=u^{1} \frac{d}{d x^{1}}+u^{2} \frac{d}{d x^{2}}
$$

we thus arrive at the active scalar system

$$
\begin{aligned}
\partial_{t} \theta+u^{1} \partial_{1} \theta+u^{2} \partial_{2} \theta & =0 \\
u^{1} & =\partial_{2}\left(A_{0} \theta\right) \\
u^{2} & =-\partial_{1}\left(A_{0} \theta\right) .
\end{aligned}
$$

As noted in the introduction, the SQG equation corresponds to the case $A_{0}=\Delta^{-1 / 2}$. We now construct an operator $A_{0}$ which will behave like ${ }^{16}$ a non-self-adjoint variant of $\Delta^{-1 / 2}$, as follows. We will need some cutoff functions:

- A Littlewood-Paley type cutoff $\gamma: \mathbb{R} \rightarrow \mathbb{R}$ which is smooth, non-negative, supported on $[1 / 2,2]$, and obeys the identity

$$
\sum_{j \in \mathbb{Z}} \gamma\left(2^{j} x\right)=1
$$

for all $x>0$;

\footnotetext{
16 In fact, it will be almost be a pseudodifferential operator in the exotic symbol class $S_{1,1}^{-1}$, as defined in [62, Chapter VII], in that the symbol obeys a large but finite number of the estimates required for this class. We will not prove or use this fact here.
} 
- A smooth function $\psi: \mathbb{R} \rightarrow \mathbb{R}$ supported on $[-20,20]$ that equals 1 on $[-10,10]$ and obeys the moment conditions $\int_{\mathbb{R}} \psi(x) P(x) d x=0$ for all polynomials $P: \mathbb{R} \rightarrow \mathbb{R}$ of degree at most 1000

- A smooth function $\varphi: \mathbb{R}^{2} \rightarrow \mathbb{R}$ supported on $B_{\mathbb{R}^{2}}((0,10), 1) \cup B_{\mathbb{R}^{2}}((0,-10), 1)$ such that $\int_{B_{\mathbb{R}^{2}}((0,10), 1)} \varphi(x) d x=1$, but such that $\int_{\mathbb{R}^{2}} \varphi(x) P(x) d x=0$ for all polynomials $P: \mathbb{R} \rightarrow \mathbb{R}$ of degree at most 1000 .

It is not difficult to construct examples of such cutoff functions $\gamma, \psi, \varphi$. The moment conditions on $\psi, \varphi$ will not be needed in this section, but will become useful in Sections 8,9 , when verifying that certain vector potential operators $A$ constructed using these functions are reasonable.

Let $M \geqslant 1$ be a sufficiently large constant (depending on $\gamma, \psi, \varphi$ ). The operator $A_{0}$ will now be defined for locally integrable $\theta$ as

$$
A_{0}(\theta)\left(x^{1}, x^{2}\right):=\frac{2}{M} \sum_{k=0}^{\infty} 2^{2 k} x^{1} \gamma\left(2^{k} x^{2}\right) \psi\left(2^{k} x^{1}\right) \int_{\mathbb{R}^{2}} \theta(y) \varphi\left(2^{k} y\right) d \operatorname{vol}(y)
$$

Note that if $\theta$ is supported on the upper half-plane $\left\{\left(x^{1}, x^{2}\right): x^{2} \geqslant 0\right\}$, then the value of $A_{0}(\theta)$ near $\left(0,2^{-k}\right)$ for some $k \geqslant 0$ is mostly driven by the behaviour of $\theta$ near $\left(0,10 \times 2^{-k}\right)$. This is analogous to how, in the previous section, the value of the velocity field $u$ at a position $x$ was driven by the active scalar $\theta$ at position $2 x$. Roughly speaking, the operator $A_{0}$ is normalised so that it will produce a downward velocity $\left(\partial_{2} A_{0} \theta,-\partial_{1} A_{0} \theta\right)$ of $\left(0,-\frac{2}{M}\right)$ near $\left(0,2^{-k}\right)$ whenever the active scalar $\theta$ is equal to 1 near $\left(0,10 \times 2^{-k}\right)$.

We now have the following blowup result:

Proposition 6.1 (Finite time blowup). Let $\theta_{0}: \mathbb{R}^{2} \rightarrow \mathbb{R}$ be smooth, compactly supported, vanishing in the half-plane $\left\{\left(x^{1}, x^{2}\right): x^{2} \leqslant 0\right\}$, and equal to 1 on the trapezoid $R:=\left\{\left(x^{1}, x^{2}\right): \frac{1}{2 M} \leqslant x^{2} \leq 100 ;\left|x^{1}\right| \leqslant x^{2}\right\}$. Then there does not exist continuously differentiable and compactly supported fields $\theta, u^{1}, u^{2}:[0,1] \times \mathbb{R}^{2} \rightarrow \mathbb{R}$ solving (6.1), (6.2), (6.3) with $\theta(0)=\theta_{0}$.

We now prove this proposition. Let $\theta_{0}$ be as in the proposition, and suppose for contradiction that such fields $\theta, u^{1}, u^{2}$ exist. From (6.5) we see that for all $0 \leqslant t \leqslant 1$, $A_{0}(\theta(t))$ vanishes on the half-space $\left\{\left(x^{1}, x^{2}\right): x^{2}<0\right\}$, so by (6.2), (6.3) we conclude that the velocity fields $u^{1}(t), u^{2}(t)$ do also; from (6.1) and the vanishing of $\theta_{0}$ we conclude that $\theta(t)$ also vanishes here. By continuity we thus have

$$
\theta\left(t, x^{1}, x^{2}\right)=0
$$

for all $0 \leqslant t \leqslant 1$ and $x^{2} \leqslant 0$.

To obtain the required contradiction, we again use the barrier method. For each time $t \in[0,1]$, let $\Omega(t) \subset \mathbb{R}^{2}$ denote the truncated hyperbolic region

$$
\Omega(t):=\left\{\left(x^{1}, x^{2}\right): \sqrt{\left((1+t) x^{1}\right)^{2}+\left(\frac{1-t}{M}\right)^{2}} \leqslant x^{2} \leqslant 20\right\}
$$




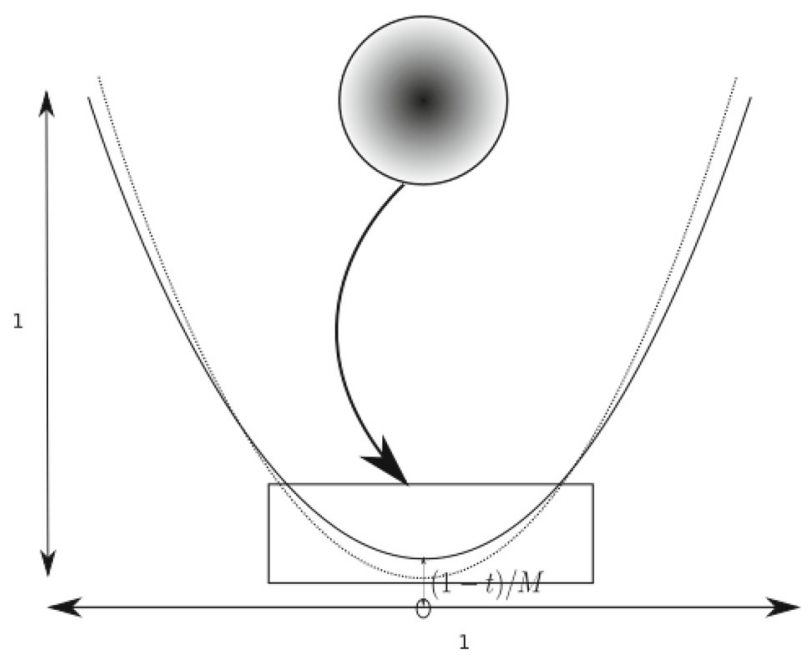

Fig. 5 A schematic depiction of $\Omega(t)$ (the region above the solid hyperboa, with the upper boundary $x^{2}=20$ out of view), together with a slightly later version $\Omega(t+d t)$ (the region above the dotted hyperbola). The origin $(0,0)$ is marked by a small circle. The rectangle and large disk represent the supports of the two functions $\left(x^{1}, x^{2}\right) \mapsto x^{1} \gamma\left(2^{k} x^{2}\right) \psi\left(2^{k} x^{1}\right)$ and $y \mapsto \varphi\left(2^{k} y\right)$ respectively that occur in (6.5), for the value of $k$ that is of most importance at the time $t$. (Actually, $y \mapsto \varphi\left(2^{k} y\right)$ also has a component supported below the $x^{1}$ axis, but this component will not be of relevance since $\theta$ vanishes there thanks to (6.6).) The active scalar $\theta(t)$ is known to equal one on $\Omega(t)$, and to vanish at and below the $x^{1}$ axis. The curved arrow signifies the one-way causality of the non-self-adjoint operator $A_{0}$

(see Figure 5). Informally, $\Omega(t)$ describes the region where we will be able to force $\theta(t)$ to take the value of 1 . Note that as $t$ increases from zero to one, the vertex $\left(0, \frac{1-t}{M}\right)$ of this region is moving outwards (towards the origin) at a constant speed $\frac{1}{M}$, but the middle portion of the boundary (where $x^{1}, x^{2}$ are comparable to 1) is instead moving inwards due to the narrowing of the hyperbola bounding $\Omega(t)$. These dynamics are chosen to match the bounds we will be able to establish on the velocity field $u$ on the boundary of this domain.

As in the previous section, let $T_{*}$ denote the supremum of all the times $0 \leqslant T_{*} \leqslant 1$ such that $\theta(t)$ is equal to 1 on $\Omega(t)$ for all $0 \leqslant t \leqslant T_{*}$. By hypothesis, $\theta_{0}$ equals 1 on $\Omega(0)$, and so $0 \leqslant T_{*} \leqslant 1$. In fact, since $\omega_{0}$ equals 1 on a neighbourhood of $\Omega(0)$, and $\theta$ is transported by the continuous vector field $u^{1} \frac{d}{d x^{1}}+u^{2} \frac{d}{d x^{2}}$ thanks to (6.1), we have $T_{*}>0$. By continuity we see that $\theta\left(T_{*}\right)$ equals 1 on $\Omega\left(T_{*}\right)$. Since $\Omega(1)$ contains the origin, we conclude from (6.6) that $T_{*}<1$. Thus we have $0<T_{*}<1$.

As $\theta$ is transported continuously by $u:=u^{1} \frac{d}{d x^{1}}+u^{2} \frac{d}{d x^{2}}$, and $\Omega(t)$ is compact and varies continuously with $t$, there must exist a point $x_{*}=\left(x_{*}^{1}, x_{*}^{2}\right)$ on the boundary of $\Omega\left(T_{*}\right)$ which is also on the boundary of the set $\left\{x: \theta\left(T_{*}, x\right)=1\right\}$. On the other hand, from (6.5) we see that $A_{0}(\theta(t))$ is supported in the region $\left\{\left(x^{1}, x^{2}\right): x^{2} \leqslant 2\right\}$ for all $t$, and so from (6.1), (6.2), (6.3) we see that $\theta\left(t, x^{1}, x^{2}\right)=\theta_{0}\left(x^{1}, x^{2}\right)$ whenever $x^{2} \geqslant 2$ and $0 \leqslant t \leqslant 1$. Since $\theta_{0}=1$ on the trapezoid $R$, we conclude that $\theta\left(T_{*}\right)$ equals 1 in a neighbourhood of $\left\{\left(x^{1}, x^{2}\right) \in \Omega\left(T_{*}\right): x^{2} \geqslant 2\right\}$. Thus we must have $x_{*}^{2}<2$, and hence by (6.7) we have 


$$
x_{*}^{2}=\sqrt{\left(\left(1+T_{*}\right) x_{*}^{1}\right)^{2}+\left(\frac{1-T_{*}}{M}\right)^{2}} .
$$

In particular,

$$
\frac{1-T_{*}}{M} \leqslant x_{*}^{2}<2
$$

From (6.8) we have

$$
\left.\partial_{t} \sqrt{\left((1+t) x^{1}\right)^{2}+\left(\frac{1-t}{M}\right)^{2}}\right|_{t=T_{*}}=\frac{\left(1+T_{*}\right)\left(x_{*}^{1}\right)^{2}-\left(1-T_{*}\right) / M^{2}}{x_{*}^{2}}
$$

and thus $\Omega\left(T_{*}\right)$ expands outward at $\left(x_{*}^{1}, x_{*}^{2}\right)$ at velocity

$$
\frac{\left(1+T_{*}\right)\left(x_{*}^{1}\right)^{2}-\left(1-T_{*}\right) / M^{2}}{x_{*}^{2}} n^{2}
$$

where $n^{2}<0$ is the $x^{2}$ component of the outward unit normal $n$ of $\Omega\left(T_{*}\right)$ at $\left(x_{*}^{1}, x_{*}^{2}\right)$ (this expansion becomes negative for large $x_{*}^{1}$ ). Since $\theta(t)$ is equal to 1 on $\Omega(t)$ for $t \leqslant T_{*}$ and is transported by $u$, but $\theta\left(T_{*}, x^{1}, x^{2}\right)$ is not equal to 1 for $\left(x^{1}, x^{2}\right)$ arbitrarily close to $\left(x_{*}^{1}, x_{*}^{2}\right)$, we conclude (on tracing characteristics backwards in time from $T_{*}$ ) the inequality

$$
n \cdot u\left(T_{*}, x_{*}^{1}, x_{*}^{2}\right) \leqslant \frac{\left(1+T_{*}\right)\left(x_{*}^{1}\right)^{2}-\left(1-T_{*}\right) / M^{2}}{x_{*}^{2}} n^{2},
$$

that is to say the outward normal velocity cannot exceed the expansion of the barrier at $\left(T_{*}, x_{*}\right)$.

To compute the left-hand side of (6.11), we first compute $A_{0}(\theta)\left(T_{*}, x^{1}, x^{2}\right)$ for $\left(x^{1}, x^{2}\right)$ in a small neighbourhood of $\left(x_{*}^{1}, x_{*}^{2}\right)$. We expand this quantity using (6.5). From the support of $\gamma$, we need only restrict attention to those $k$ for which $2^{-k} \geqslant \frac{1}{2} x^{2}$; in particular, from (6.9) and the restriction $k \geqslant 0$ we have

$$
1 \geqslant 2^{-k} \geqslant \frac{1-T_{*}}{3 M}
$$

for $\left(x^{1}, x^{2}\right)$ sufficiently close to $\left(x_{*}^{1}, x_{*}^{2}\right)$. The function $y \mapsto \varphi\left(2^{k} y\right)$ in the integrand in (6.5) is supported in $B_{\mathbb{R}^{2}}\left(\left(0,10 \times 2^{-k}\right), 2^{-k}\right) \cup B_{\mathbb{R}^{2}}\left(\left(0,-10 \times 2^{-k}\right), 2^{-k}\right)$. By (6.6), $\theta$ vanishes on the latter ball $B_{\mathbb{R}^{2}}\left(\left(0,-10 \times 2^{-k}\right), 2^{-k}\right)$. By $(6.12)$, the ball $B_{\mathbb{R}^{2}}\left(\left(0,10 \times 2^{-k}\right), 2^{-k}\right)$ is contained in the truncated cone

$$
\left\{\left(x^{1}, x^{2}\right): 9 \times \frac{\left(1-T_{*}\right)}{3 M} \leqslant x^{2} \leqslant 11 ;\left|x^{1}\right| \leqslant \frac{1}{9} x^{2}\right\}
$$


which can be seen in turn from (6.7) and a brief calculation to lie in $\Omega\left(T_{*}\right)$. By construction of $T_{*}$ and continuity, we have $\theta\left(T_{*}, y\right)=1$ for all $y$ in $B_{\mathbb{R}^{2}}((0,10 \times$ $\left.\left.2^{-k}\right), 2^{-k}\right)$, and hence

$$
\begin{aligned}
\int_{\mathbb{R}^{2}} \theta(y) \varphi\left(2^{k} y\right) d \operatorname{vol}(y) & =\int_{B_{\mathbb{R}^{2}}\left(\left(0,10 \times 2^{-k}\right), 2^{-k}\right)} \varphi\left(2^{k} y\right) d \operatorname{vol}(y) \\
& =2^{-2 k} \int_{B_{\mathbb{R}^{2}}((0,10), 1)} \varphi(y) d \operatorname{vol}(y) \\
& =2^{-2 k}
\end{aligned}
$$

by construction of $\varphi$. Inserting this into (6.5), we conclude that

$$
A_{0}(\theta)\left(T_{*}, x^{1}, x^{2}\right)=\frac{2}{M} \sum_{k=0}^{\infty} x^{1} \gamma\left(2^{k} x^{2}\right) \psi\left(2^{k} x^{1}\right)
$$

From (6.8) we have $\left|x_{*}^{1}\right| \leqslant x_{*}^{2}$, and hence

$$
\left|x^{1}\right| \leqslant 2\left|x^{2}\right|
$$

for $\left(x^{1}, x^{2}\right)$ sufficiently close to $\left(x_{*}^{1}, x_{*}^{2}\right)$. From the construction of $\gamma$ and $\psi$, we conclude that $\psi\left(2^{k} x^{1}\right)$ equals 1 whenever $\gamma\left(2^{k} x^{2}\right)$ is non-zero. Thus

$$
A_{0}(\theta)\left(T_{*}, x^{1}, x^{2}\right)=\frac{2}{M} x^{1} \sum_{k=0}^{\infty} \gamma\left(2^{k} x^{2}\right)
$$

If $x_{*}^{2} \leqslant 1 / 2$, then the constraint $k \geqslant 0$ can be dropped, and from (6.4) we thus have $A_{0}(\theta)\left(x^{1}, x^{2}\right)=\frac{2}{M} x^{1}$. From (6.2), (6.3) we thus have

$$
u\left(T_{*}, x_{*}^{1}, x_{*}^{2}\right)=\left(0,-\frac{2}{M}\right),
$$

and hence by (6.11) and the negativity of $n^{2}$

$$
\frac{2}{M} \leqslant \frac{\left(1-T_{*}\right) / M^{2}-\left(1+T_{*}\right)\left(x_{*}^{1}\right)^{2}}{x_{*}^{2}} .
$$

But this contradicts (6.9) (discarding the negative term $\left.\left(1+T_{*}\right)\left(x_{*}^{1}\right)^{2}\right)$. Thus we must have $x_{*}^{2} \geqslant 1 / 2$. But then the quantity $\gamma\left(2^{k} x^{2}\right)$ only is non-zero for $k=0,1$. Meanwhile, from (6.9), (6.8) we have

$$
1 \lesssim\left|x_{*}^{1}\right|, x_{*}^{2} \lesssim 1
$$


From (6.13), (6.2), (6.3) we then have the crude bounds

$$
u\left(T_{*}, x_{*}^{1}, x_{*}^{2}\right)=O(1 / M)
$$

From (6.11) we thus have

$$
\left(-n^{2}\right) \frac{\left(1+T_{*}\right)\left(x_{*}^{1}\right)^{2}-\left(1-T_{*}\right) / M^{2}}{x_{*}^{2}} \leqslant O(1 / M) .
$$

On the other hand, from (6.15) we have for $M$ large enough that $-n^{2} \gtrsim 1$ and $\frac{\left(1+T_{*}\right)\left(x_{*}^{1}\right)^{2}-\left(1-T_{*}\right) / M^{2}}{x_{*}^{2}} \gtrsim 1$, giving the required contradiction. This concludes the proof of Proposition 6.1.

\section{A Stable, Non-self-adjoint Blowup}

In this section we prove Theorem 1.11, using a three-dimensional variant of the argument ${ }^{17}$ used to prove Proposition 6.1. We will need a large constant $M>1$ to be chosen later. Now we select initial data $\omega_{0} \in B_{2}\left(\mathbb{R}^{3}\right)$ with the following properties:

- $\omega_{0}$ is smooth and compactly supported. When restricted to the ball $B_{\mathbb{R}^{3}}(0,100 M)$, $\omega_{0}$ supported on the cylindrical region $\left\{\left(x^{1}, x^{2}, x^{3}\right) \in B_{\mathbb{R}^{3}}(0,100 M):\left(x^{1}\right)^{2}+\right.$ $\left.\left(x^{2}\right)^{2} \leqslant \frac{1}{M}\right\}$.

- For any $-50 M \leqslant x^{3} \leqslant 50 M$, one has the constant circulation

$$
\int_{\left\{\left(x_{1}, x_{2}, x_{3}\right):\left(x^{1}\right)^{2}+\left(x^{2}\right)^{2} \leqslant \frac{1}{M}\right\}} \omega_{0}=1
$$

where we give the disk $\left\{\left(x^{1}, x^{2}, x^{3}\right):\left(x^{1}\right)^{2}+\left(x^{2}\right)^{2} \leqslant \frac{1}{M}\right\}$ the orientation of $\frac{d}{d x^{1}} \wedge \frac{d}{d x^{2}}$.

To create such an $\omega_{0}$, one can for instance set $\omega_{0}=d \lambda$, where $\lambda \in C_{c}^{\infty} \cap \Lambda_{1}\left(\mathbb{R}^{3}\right)$ is chosen to be equal to the closed form

$$
\lambda=\frac{1}{2 \pi} \frac{x^{1} d x^{2}-x^{2} d x^{1}}{\left(x^{1}\right)^{2}+\left(x^{2}\right)^{2}}
$$

in the region $\left\{\left(x^{1}, x^{2}, x^{3}\right) \in B_{\mathbb{R}^{3}}(0,100 M):\left(x^{1}\right)^{2}+\left(x^{2}\right)^{2} \geqslant \frac{1}{M}\right\}$, but otherwise arbitrary outside of this region; the constant circulation (7.1) then follows from Stokes' theorem.

Next, we construct the vector potential operator $A$. We introduce the cylindrically radial variable

$$
r:=\sqrt{\left(x^{1}\right)^{2}+\left(x^{2}\right)^{2}}
$$

\footnotetext{
17 A simplified version of this argument, involving a non-compactly supported initial vorticity $\omega_{0}$, can be found at terrytao.wordpress.com/2016/02/01.
} 
and the associated cylindrically radial vector field

$$
\frac{d}{d r}:=\frac{x^{1}}{r} \frac{d}{d x^{1}}+\frac{x^{2}}{r} \frac{d}{d x^{2}}
$$

defined away from the $x^{3}$ axis $\left\{\left(0,0, x^{3}\right): x^{3} \in \mathbb{R}\right\}$. Our construction will be designed so that the velocity field $u=\delta A \omega$ will be equal to the inward cylindrically radial vector field

$$
-\frac{1}{M r} \frac{d}{d r}=\frac{-x^{1} \frac{d}{d x^{1}}-x^{2} \frac{d}{d x^{2}}}{M r^{2}}
$$

in a certain key portion of physical space $\mathbb{R}^{3}$. Observe that away from the $x^{3}$-axis, this field is divergence free, and can be written in turn as a divergence

$$
-\frac{1}{M r} \frac{d}{d r}=\delta \frac{-x^{1} x^{3} \frac{d}{d x^{1}} \wedge \frac{d}{d x^{3}}-x^{2} x^{3} \frac{d}{d x^{2}} \wedge \frac{d}{d x^{3}}}{M r^{2}} .
$$

For technical reasons (having to do with ensuring that the vector potential operator $A$ we will construct is reasonable), we need to replace the 2-vector field $\frac{-x^{1} x^{3} \frac{d}{d x^{1}} \wedge \frac{d}{d x^{3}}-x^{2} x^{3} \frac{d}{d x^{2}} \wedge \frac{d}{d x^{3}}}{r^{2}}$ appearing on the right-hand side of (7.2) by a variant $\alpha$ that enjoys better moment vanishing conditions. More precisely, by inserting a suitable cutoff in the angular variable, one can find a 2-vector field $\alpha \in \Gamma^{2}\left(\mathbb{R}^{3}\right)$ that is smooth away from the origin and homogeneous of degree zero, such that

$$
\alpha=\frac{-x^{1} x^{3} \frac{d}{d x^{1}} \wedge \frac{d}{d x^{3}}-x^{2} x^{3} \frac{d}{d x^{2}} \wedge \frac{d}{d x^{3}}}{r^{2}}
$$

and hence

$$
\delta \alpha=-\frac{1}{r} \frac{d}{d r}
$$

in the exterior cone region $\left\{\left(x^{1}, x^{2}, x^{3}\right): r>\left|x^{3}\right|\right\}$, and such that all moments of $\alpha$ vanish to order 1000 (say) on each sphere, or in other words the three components $\alpha^{12}, \alpha^{13}, \alpha^{23}$ of $\alpha$ are such that

$$
\int_{S^{2}} \alpha^{i j}(\theta) P(\theta) d \theta=0
$$

for all polynomials $P: \mathbb{R}^{3} \rightarrow \mathbb{R}$ of degree at most 1000 , where $S^{2}$ is the unit sphere in $\mathbb{R}^{3}$ and $d \theta$ denotes surface measure.

We introduce a smooth dyadic partition of unity of Littlewood-Paley type, writing

$$
1=\sum_{k \in \mathbb{Z}} \psi\left(2^{k} x\right)
$$


for a suitable smooth, spherically symmetric function $\psi: \mathbb{R}^{3} \rightarrow \mathbb{R}$ (not depending on $M)$ supported on the annulus $\{x: 1 / 2 \leqslant|x| \leqslant 2\}$. Clearly we can then decompose $\alpha=\sum_{k \in \mathbb{Z}} \alpha_{k}$, where $\alpha_{k} \in C_{c}^{\infty} \cap \Gamma^{2}\left(\mathbb{R}^{3}\right)$ is defined by the formula

$$
\alpha_{k}(x):=\alpha(x) \psi\left(2^{k} x\right)
$$

Next, we let $\varphi: \mathbb{R}^{3} \rightarrow \mathbb{R}$ be a smooth compactly supported function (not depending on $M$ ) of the form

$$
\varphi\left(x^{1}, x^{2}, x^{3}\right)=\varphi_{12}\left(x^{1}, x^{2}\right) \varphi_{3}\left(x^{3}\right)
$$

where $\varphi_{12}: \mathbb{R}^{2} \rightarrow \mathbb{R}$ is a smooth spherically symmetric function supported on the disk $B_{\mathbb{R}^{2}}(0,20)$ that equals one on the disk $B_{\mathbb{R}^{2}}(0,10)$ and obeys the moment conditions

$$
\int_{\mathbb{R}^{2}} \varphi_{12}\left(x^{1}, x^{2}\right) P\left(x^{1}, x^{2}\right) d \operatorname{vol}(x)=0
$$

for any polynomial $P$ of degree at most 1000 , and $\varphi_{3}: \mathbb{R} \rightarrow \mathbb{R}$ is a smooth function supported on $[1,2]$ with the normalisation

$$
\int_{1}^{2} \varphi_{3}\left(x^{3}\right) d x^{3}=1
$$

We define the vector potential operator $A$ by the formula

$$
A \omega(x):=\sum_{k=0}^{\infty} \frac{2^{k}}{M^{2}} \alpha_{k}(x) \int_{\mathbb{R}^{3}} \omega_{12}(y) \varphi\left(2^{k} y / M\right) d \operatorname{vol}(y)
$$

where $\omega_{12}$ is the $d x^{1} \wedge d x^{2}$ component of $\omega$. This operator $A$ is designed so that $A \omega$ will equal $\frac{1}{M} \alpha$ in regions where $\omega$ has circulation equal to one. In particular, $u=\delta A \omega$ will equal $-\frac{1}{M r} \frac{d}{d r}$ in these regions.

It is easy to see that the sum defining $A \omega$ is absolutely convergent for $\omega \in C_{c}^{\infty} \cap$ $B_{2}\left(\mathbb{R}^{3}\right)$. One can write $A$ as an integral operator

$$
A \omega(x)=\int_{\mathbb{R}^{3}} K(x, y) \omega_{12}(y) d \operatorname{vol}(y)
$$

where the kernel $K$ is given by the formula

$$
K(x, y)=\sum_{k=0}^{\infty} \frac{2^{k}}{M^{2}} \alpha \psi\left(2^{k} x\right) \varphi\left(2^{k} y / M\right)
$$

(here we exploit the hypothesis that $\alpha$ is homogeneous of degree zero). Since $\alpha \psi, \varphi$ are smooth and compactly supported, we see that $K$ obeys the bounds (1.19) for all $0 \leqslant i, j \leqslant 100$ (with implied constants depending on $M$ ); indeed, one can even replace the quantity $|x-y|$ in (1.19) by the larger quantity $2 \max (|x|,|y|)$, and obtain 
bounds for arbitrary $i, j \geqslant 0$ if one allows the implied constant to depend on these parameters. Now we show (1.20) (again with bounds depending on $M$ ). It will suffice to establish the slightly stronger bounds

$$
\|A \omega\|_{\dot{H}^{r+2}(\mathcal{M})} \lesssim M\|\omega\|_{\dot{H}^{r} k(\mathcal{M})}
$$

for all $0 \leqslant r \leqslant 100$. By duality, it suffices to establish the bounds

$$
\int_{\mathbb{R}^{3}}\langle\beta(x), A \omega(x)\rangle d \operatorname{vol}(x) \lesssim M\|\omega\|_{\dot{H}^{s}\left(\mathbb{R}^{3}\right)}\|\beta\|_{\dot{H}^{-s-2}\left(\mathbb{R}^{3}\right)}
$$

for any $\beta \in \dot{H}^{-s-2} \cap \Lambda_{2}\left(\mathbb{R}^{3}\right)$ and any $0 \leqslant s \leqslant 100$. By Littlewood-Paley decomposition and Schur's test, it suffices to show that

$$
\int_{\mathbb{R}^{3}}\langle\beta(x), A \omega(x)\rangle d \operatorname{vol}(x) \lesssim M \min \left(N_{1}^{-2}, N_{1}^{200} N_{2}^{-202}\right)\|\omega\|_{L^{2}\left(\mathbb{R}^{3}\right)}\|\beta\|_{L^{2}\left(\mathbb{R}^{3}\right)}
$$

whenever $\omega, \beta \in C_{c}^{\infty} \cap \Lambda_{2}\left(\mathbb{R}^{3}\right)$ have Fourier transforms supported on the annuli $\left\{\xi:|\xi| \sim N_{1}\right\}$ and $\left\{\eta:|\eta| \sim N_{2}\right\}$ respectively for some $N_{1}, N_{2}>0$. The left-hand side may be expanded as $\sum_{k=0}^{\infty} \frac{2^{k}}{M^{2}} X_{k} Y_{k}$, where

$$
X_{k}:=\int_{\mathbb{R}^{3}}\left\langle\beta(x), \alpha \psi\left(2^{k} x\right)\right\rangle d \operatorname{vol}(x)
$$

and

$$
Y_{k}:=\int_{\mathbb{R}^{3}} \omega_{12}(y) \varphi\left(2^{k} y / M\right) d \operatorname{vol}(y) .
$$

From the smoothness and moment conditions on $\alpha \psi$, the Parseval identity, and Cauchy-Schwarz, we see that

$$
X_{k} \lesssim \min \left(N_{1} / 2^{k}, 2^{k} / N_{1}\right)^{300} 2^{-3 k / 2}\|\beta\|_{L^{2}\left(\mathbb{R}^{3}\right)}
$$

for any $k$; similarly

$$
Y_{k} \lesssim M \min \left(N_{2} / 2^{k}, 2^{k} / N_{2}\right)^{300} 2^{-3 k / 2}\|\omega\|_{L^{2}\left(\mathbb{R}^{3}\right)} .
$$

Inserting these bounds and summing in $k$, one obtains the claim.

We can now prove Theorem 1.11 with this choice of $\omega_{0}$ and $A$. Suppose for contradiction that there is a solution $\omega \in X^{10,2}, u \in Y^{10,2}$ to (1.10), (1.11) with $s=10$ on the time interval $[0,1]$. This is enough regularity to interpret the equations $(1.10)$, (1.11) in the classical sense. The velocity $u$ is bounded in $\mathbb{R}^{3} \times[0,1]$, and the vorticity $\omega$ is transported by $u$ and is compactly supported at time zero, and is thus compactly supported in all of $\mathbb{R}^{3} \times[0,1]$. From (7.7), (1.11) we see that $u$ is supported in the ball $B_{\mathbb{R}^{3}}(0,2)$, and thus by (1.11) $\omega$ is stationary outside of this ball. 


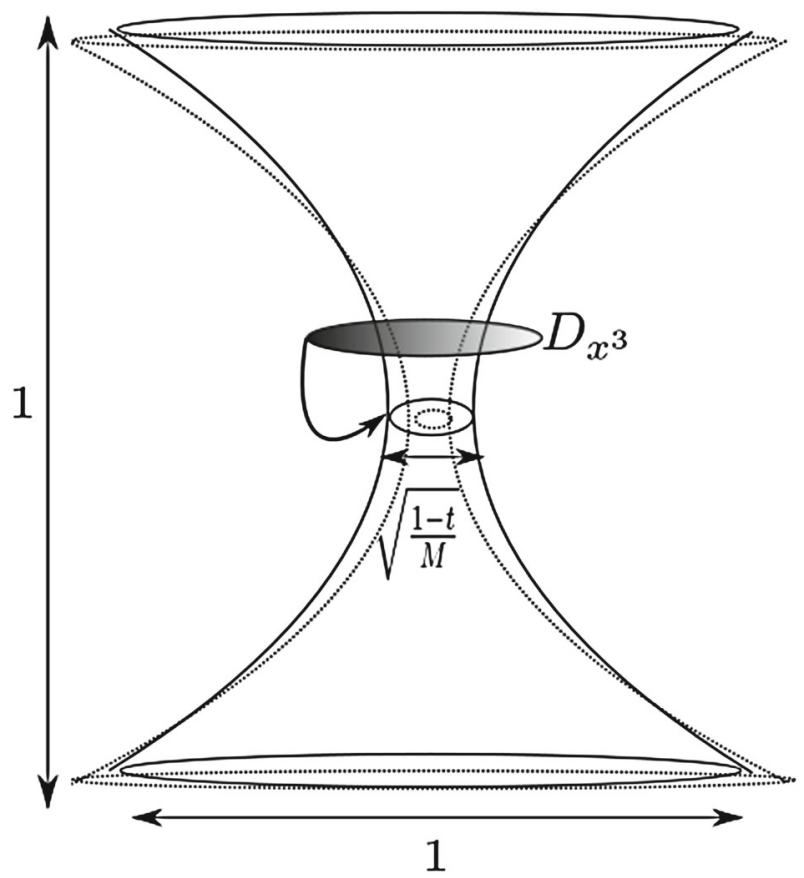

Fig. 6 A schematic depiction of $\Omega(t)$ (the region inside the hyperboloid, with the exterior of $B_{\mathbb{R}^{3}}(0,50 M)$ out of view), together with a slightly later version $\Omega(t+d t)$ (the region inside the dotted hyperboloid). The origin $(0,0)$ is marked by a small circle. The vorticity $\omega$ is supported inside $\Omega(t)$, which allows one to use the Kelvin circulation theorem to calculate the circulation on disks $D_{x^{3}}$ such as the shaded one depicted here. The curved arrow depicts the causal relationship in the non-self-adjoint vector potential $A$, which uses the circulation on disks such as $D_{x^{3}}$ to determine the velocity field in the "neck" of the hyperboloid

We once again use the barrier method. For any time $0 \leqslant t \leqslant 1$, let $\Omega(t) \subset \mathbb{R}^{3}$ denote the region

$$
\Omega(t):=\left(\mathbb{R}^{3} \backslash B_{\mathbb{R}^{3}}(0,50 M)\right) \cup\left\{\left(x^{1}, x^{2}, x^{3}\right) \in \mathbb{R}^{3}: r \leqslant \sqrt{\frac{1-t}{M}+(1+t)\left(x^{3}\right)^{2}}\right\}
$$

inside the ball $B_{\mathbb{R}^{3}}(0,50 M)$; this is the interior of a one-sheeted hyperboloid which pinches at the spatial origin $(0,0,0)$ at time $t=1$, while simultaneously becoming slightly wider away from this origin. See Figure 6. From the construction of $\omega_{0}$, we see that $\omega_{0}$ is supported in $\Omega(0)$; from continuity and the fact that the support of $\omega$ propagates at bounded speed, we see also that $\omega(t)$ is supported in $\Omega(t)$ for sufficiently small $t$. Let $T_{*}$ be the supremum of all times $0 \leqslant T_{*} \leqslant 1$ for which $\omega(t)$ is supported in $\Omega(t)$ for all $0 \leqslant t \leqslant T_{*}$, then from the previous observation we have $0<T_{*} \leqslant 1$, and from continuity $\omega\left(T_{*}\right)$ is supported in $\Omega\left(T_{*}\right)$. We now claim the circulation identity

$$
\int_{D_{x^{3}}} \omega(t)=1
$$


on the disk $D_{x^{3}}:=\left\{\left(x^{1}, x^{2}, x^{3}\right): r \leqslant 30 M\right\}$ for all $-10 M \leqslant x^{3} \leqslant 10 M$ and $0 \leqslant t \leqslant T_{*}$. For $t=0$, this follows from the construction of $\omega_{0}$. The set of $0 \leqslant t \leqslant T_{*}$ for which the above bound holds is clearly closed in $t$. Finally, if the above bound holds for some $0 \leqslant t<1$, and $t^{\prime}$ is a time slightly larger than $t$, then from conservation of circulation we have

$$
\int_{S} \omega\left(t^{\prime}\right)=1
$$

where $S$ is the image of the disk $D_{x^{3}}$ after flowing along the velocity field $u$ from time $t$ to time $t^{\prime}$. But if $t^{\prime}$ is sufficiently close to $t, S$ is homologous to $D_{x^{3}}$ up to a thin annular strip outside of $\Omega\left(t^{\prime}\right)$, and so from Stokes theorem and the closed nature of $\omega$ we conclude that (7.9) holds for all $t^{\prime}$ slightly larger than $t$, and from a continuity argument we conclude that (7.9) holds for all $0 \leqslant t \leqslant T_{*}$.

We can now exclude the case $T_{*}=1$, since in this case $\Omega(1)$ degenerates to a cone that only intersects the disk $D_{0}=\left\{\left(x^{1}, x^{2}, 0\right): r \leqslant 30 M\right\}$ at the origin $(0,0,0)$, contradicting (7.9) and the regularity hypotheses on $\omega$. Thus we have $0<T_{*}<1$.

By definition of $T_{*}$, and the continuity of $\omega$, there must be a point $x_{*}=\left(x_{*}^{1}, x_{*}^{2}, x_{*}^{3}\right)$ on the boundary of $\Omega\left(T_{*}\right)$ which is on the boundary of the support of $\omega\left(T_{*}\right)$. Since $\omega\left(T_{*}\right)$ is equal to $\omega_{0}$ outside of $B_{\mathbb{R}^{3}}(0,2)$, and $\omega_{0}$ vanishes near the boundary of $\Omega\left(T_{*}\right)$, we must have $x_{*} \in B_{\mathbb{R}^{3}}(0,2)$. From (7.8) we conclude that the radial coordinate $r_{*}:=\sqrt{\left(x_{*}^{1}\right)^{2}+\left(x_{*}^{2}\right)^{2}}$ is given by

$$
r_{*}=\sqrt{\frac{1-T}{M}+(1+T)\left(x_{*}^{3}\right)^{2}}
$$

which implies in particular that

$$
\max \left(\left|x_{*}^{3}\right|, \sqrt{\frac{1-T_{*}}{M}}\right) \leqslant r \leqslant 2 \max \left(\left|x_{*}^{3}\right|, \sqrt{\frac{1-T_{*}}{M}}\right)
$$

and hence by Pythagoras' theorem

$$
\max \left(\left|x_{*}^{3}\right|, \sqrt{\frac{1-T_{*}}{M}}\right) \leqslant\left|x_{*}\right| \leqslant 3 \max \left(\left|x_{*}^{3}\right|, \sqrt{\frac{1-T_{*}}{M}}\right) .
$$

On the other hand, if $n$ denotes the outward normal to $\Omega\left(T_{*}\right)$ at $x_{*}$, then since

$$
\begin{aligned}
\left.\frac{d}{d t} \sqrt{\frac{1-t}{M}+(1+t)\left(x_{*}^{3}\right)^{2}}\right|_{t=T_{*}} & =\frac{\left(x_{*}^{3}\right)^{2}-\frac{1}{M}}{2} \frac{1}{\sqrt{\frac{1-T_{*}}{M}+\left(1+T_{*}\right)\left(x_{*}^{3}\right)^{2}}} \\
& =-\frac{\frac{1}{M}-\left(x_{*}^{3}\right)^{2}}{2 r_{*}}
\end{aligned}
$$


we see that at $x_{*}, \Omega\left(T_{*}\right)$ is moving outwards at speed

$$
\frac{\left(x_{*}^{3}\right)^{2}-\frac{1}{M}}{2 r_{*}} n^{r}
$$

where $n^{r}$ is the radial component of $n$ (note this component is negative, reflecting inwards motion, when $x^{3}$ is small). Since $\omega$ is transported by $u$ and is supported on $\Omega(t)$ for all $t \leqslant T_{*}$, we thus have

$$
n \cdot u\left(T_{*}, x_{*}\right) \geqslant \frac{\left(x_{*}^{3}\right)^{2}-\frac{1}{M}}{2 r_{*}} n^{r} .
$$

Now we compute the velocity field $u\left(T_{*}, x_{*}\right)$ at $\left(T_{*}, x_{*}\right)$. By (1.11), (7.7) we have

$$
u\left(T_{*}, x_{*}\right):=\sum_{j=0}^{\infty} \frac{2^{j}}{M^{2}} \delta \alpha_{j}\left(x_{*}\right) \int_{\mathbb{R}^{3}} \omega_{12}(T, y) \varphi\left(2^{j} y / M\right) d \operatorname{vol}(y) .
$$

The quantity $\delta \alpha_{k}\left(x_{*}\right)$ is only non-vanishing when

$$
2^{-k-1} \leqslant\left|x_{*}\right| \leqslant 2^{-k+1},
$$

so we may restrict to $k$ obeying these bounds. By (7.4), the function $\varphi\left(2^{k} y / M\right)$ is only non-vanishing when

$$
2^{-k} M \leqslant y^{3} \leqslant 2^{-k+1} M
$$

and

$$
r(y) \leqslant 20 M 2^{-k}
$$

where $r(y):=\sqrt{\left(y^{1}\right)^{2}+\left(y^{2}\right)^{2}}$ is the cylindrically radial component of $y$. In particular $|y| \leqslant 30 M$ since $k \geq 0$. Since $\omega\left(T_{*}\right)$ is supported in $\Omega\left(T_{*}\right)$, we conclude that $\omega_{12}(T, y) \varphi\left(2^{k} y / M\right)$ is only non-vanishing when

$$
r(y) \leqslant \sqrt{\frac{1-T_{*}}{M}+\left(1+T_{*}\right)\left(y^{3}\right)^{2}}
$$

which implies from the triangle inequality that

$$
r(y) \leqslant \sqrt{\frac{1-T_{*}}{M}}+2\left|y^{3}\right| .
$$

Using (7.15), (7.14), (7.11) we have

$$
y^{3} \geqslant \frac{1}{2} M\left|x_{*}\right| \geqslant \frac{1}{2} M \sqrt{\frac{1-T_{*}}{M}}
$$


and hence by (7.15)

$$
r(y) \leqslant 3 y^{3} \leqslant 6 M 2^{-k}
$$

Using (7.4), we then have

$$
\varphi\left(2^{k} y / M\right)=\varphi_{3}\left(2^{k} y^{3} / M\right)
$$

and thus

$$
\int_{\mathbb{R}^{3}} \omega_{12}\left(T_{*}, y\right) \varphi\left(2^{k} y / M\right) d \operatorname{vol}(y)=\int_{\mathbb{R}} \varphi_{3}\left(2^{k} y^{3} / M\right)\left(\int_{r(y) \leqslant 30 M} \omega_{12}\left(T_{*}, y\right) d y^{1} d y^{2}\right) d y^{3}
$$

Applying (7.9) and (7.6), the right-hand side evaluates to $M / 2^{k}$. From (7.13) we conclude that

$$
u\left(T_{*}, x_{*}\right):=\frac{1}{M} \sum_{k=0}^{\infty} \delta \alpha_{k}\left(x_{*}\right)
$$

Suppose first that $\left|x_{*}\right| \leqslant 1 / 2$, then $\delta \alpha_{k}$ vanishes for $k<0$, and we conclude from (7.3) that $u\left(T_{*}, x_{*}\right)$ is the inward vector field

$$
u\left(T_{*}, x\right)=-\frac{1}{M r_{*}} \frac{d}{d r}
$$

and hence

$$
n \cdot u\left(T_{*}, x_{*}\right)=-\frac{1}{M r_{*}} n^{r}
$$

Since $n^{r}$ is positive, this contradicts (7.12). Thus we must have $1 / 2 \leqslant\left|x_{*}\right| \leqslant 2$, which from (7.11) implies that $\left|x_{3}\right|$ is comparable to 1 . Now we use the boundedness of $\alpha$ and its derivatives on this annulus to obtain the crude bound

$$
\begin{gathered}
u\left(T_{*}, x_{*}\right)=O(1 / M) \\
n \cdot u\left(T_{*}, x_{*}\right)=O(1 / M) .
\end{gathered}
$$

On the other hand, in the region $1 / 2 \leqslant\left|x_{*}\right| \leqslant 2$, one checks from (7.8) that $n^{r}$ is comparable to 1 , and this again contradicts (7.12) for $M$ large enough. This concludes the proof of Theorem 1.11. 


\section{Embedding SQG Type Equations into Euler Type Equations}

\subsection{Formal Calculations}

To motivate our proof of Theorem 1.12, we begin with the following observation that embeds solutions of SQG type equations on $\mathbb{R}^{2}$ into solutions of Euler type equations on $\mathbb{R}^{2} \times \mathbb{R} / \mathbb{Z}$; a key feature of this embedding is that the vector potential operator $\tilde{A}$ on $\mathbb{R}^{2} \times \mathbb{R} / \mathbb{Z}$ will always be formally self-adjoint, even when the vector potential operator $A$ on $\mathbb{R}^{2}$ is not. In this subsection we ignore issues of regularity or decay in calculations, proceeding instead at a purely formal level.

Let $A: B_{2}\left(\mathbb{R}^{2}\right) \rightarrow \Gamma^{2}\left(\mathbb{R}^{2}\right)$ be a (formal) vector potential operator on $\mathbb{R}^{2}$. We can write this operator in coordinates as

$$
A\left(\theta d x^{1} \wedge d x^{2}\right)=\left(A_{0} \theta\right) \frac{d}{d x^{1}} \wedge \frac{d}{d x^{2}}
$$

for all scalar functions $\theta: \mathbb{R}^{2} \rightarrow \mathbb{R}$, and some linear operator $A_{0}: \Lambda_{0}\left(\mathbb{R}^{2}\right) \rightarrow \Lambda_{0}\left(\mathbb{R}^{2}\right)$. Let $\omega, u$ solve the generalised Euler equations with vector potential operator $A$; writing in coordinates

$$
\omega=\theta d x^{1} \wedge d x^{2}
$$

and

$$
u=u^{1} \frac{d}{d x^{1}}+u^{2} \frac{d}{d x^{2}}
$$

we thus arrive at the active scalar system (6.1), (6.2), (6.3) from Section 6. We can formally define the adjoint $A_{0}^{*}: \Lambda_{0}\left(\mathbb{R}^{2}\right) \rightarrow \Lambda_{0}\left(\mathbb{R}^{2}\right)$ of $A_{0}$ by requiring the formal identity

$$
\int_{\mathbb{R}^{2}}\left(A_{0} \theta\right)(x) \theta^{\prime}(x) d \operatorname{vol}(x)=\int_{\mathbb{R}^{2}} \theta(x)\left(A_{0}^{*} \theta^{\prime}\right)(x) d \operatorname{vol}(x)
$$

for all $\theta, \theta^{\prime} \in \Lambda_{0}\left(\mathbb{R}^{2}\right)$.

We now suppose we have a three-dimensional extension $\tilde{A}_{0}: \Lambda_{0}\left(\mathbb{R}^{2} \times \mathbb{R} / \mathbb{Z}\right) \rightarrow$ $\Lambda_{0}\left(\mathbb{R}^{2} \times \mathbb{R} / \mathbb{Z}\right)$ of $A_{0}$, by which we mean a linear operator on $\Lambda_{0}\left(\mathbb{R}^{2} \times \mathbb{R} / \mathbb{Z}\right)$ obeying the compatibility condition

$$
\tilde{A}_{0}(\theta \circ \pi):=\left(A_{0} \theta\right) \circ \pi
$$

for all $\theta \in \Lambda_{0}\left(\mathbb{R}^{2}\right)$, where $\pi: \mathbb{R}^{2} \times \mathbb{R} / \mathbb{Z} \rightarrow \mathbb{R}^{2}$ is the projection map $\pi\left(x^{1}, x^{2}, x^{3}\right):=$ $\left(x^{1}, x^{2}\right)$. We also suppose that we have an adjoint operator $\tilde{A}_{0}^{*}: \Lambda_{0}\left(\mathbb{R}^{2} \times \mathbb{R} / \mathbb{Z}\right) \rightarrow$ $\Lambda_{0}\left(\mathbb{R}^{2} \times \mathbb{R} / \mathbb{Z}\right)$ which extends $A_{0}^{*}$ in the sense that the analogue

$$
\tilde{A}_{0}^{*}(\theta \circ \pi):=\left(A_{0}^{*} \theta\right) \circ \pi
$$


of (8.1) holds for all $\theta \in \Lambda_{0}\left(\mathbb{R}^{2}\right)$; we also assume that $\tilde{A}_{0}^{*}$ is the adjoint of $\tilde{A}_{0}$ in the sense that

$$
\int_{\mathbb{R}^{2} \times \mathbb{R} / \mathbb{Z}}\left(\tilde{A}_{0} \theta\right)(x) \theta^{\prime}(x) d \operatorname{vol}(x)=\int_{\mathbb{R}^{2}} \theta(x)\left(\tilde{A}_{0}^{*} \theta^{\prime}\right)(x) d \operatorname{vol}(x)
$$

for all $\theta, \theta^{\prime} \in \Lambda_{0}\left(\mathbb{R}^{2} \times \mathbb{R} / \mathbb{Z}\right)$. One could impose further properties on $\tilde{A}_{0}$ and $\tilde{A}_{0}^{*}$, for instance that they are invariant with respect to translations in the $x^{3}$ direction, but we will not need to do so for this formal calculation. Heuristically, if $A_{0}$ (and hence $A_{0}^{*}$ ) are pseudodifferential operators of order -1 , then we would expect to be able to select extensions $\tilde{A}_{0}, \tilde{A}_{0}^{*}$ to also be pseudodifferential operators of order -1 ; again, we will not enforce these requirements during this formal discussion.

We now formally define an operator $\tilde{A}: B_{2}\left(\mathbb{R}^{2} \times \mathbb{R} / \mathbb{Z}\right) \rightarrow \Gamma^{2}\left(\mathbb{R}^{2} \times \mathbb{R} / \mathbb{Z}\right)$ by the formula

$$
\begin{aligned}
\tilde{A} \omega:= & -\tilde{A}_{0} \Delta^{-1}\left(\partial_{1} \omega_{13}+\partial_{2} \omega_{23}\right) \frac{d}{d x^{1}} \wedge \frac{d}{d x^{2}} \\
& +\partial_{1} \Delta^{-1} \tilde{A}_{0}^{*} \omega_{12} \frac{d}{d x^{1}} \wedge \frac{d}{d x^{3}} \\
& +\partial_{2} \Delta^{-1} \tilde{A}_{0}^{*} \omega_{12} \frac{d}{d x^{2}} \wedge \frac{d}{d x^{3}} \\
& +\Delta^{-1} \omega_{13} \frac{d}{d x^{1}} \wedge \frac{d}{d x^{3}} \\
& +\Delta^{-1} \omega_{23} \frac{d}{d x^{2}} \wedge \frac{d}{d x^{3}} \\
& +\Delta^{-1} \omega_{12} \frac{d}{d x^{1}} \wedge \frac{d}{d x^{2}}
\end{aligned}
$$

whenever $\omega \in B_{2}\left(\mathbb{R}^{2} \times \mathbb{R} / \mathbb{Z}\right)$, where $\omega$ is expressed in coordinates as

$$
\omega=\omega_{12} d x^{1} \wedge d x^{2}+\omega_{13} d x^{1} \wedge d x^{3}+\omega_{23} d x^{2} \wedge d x^{3}
$$

Here we pause to make a technical remark: because there are only two noncompact dimensions in $\mathbb{R}^{2} \times \mathbb{R} / \mathbb{Z}$, the operator $\Delta^{-1}$ is not quite uniquely defined even on $C_{c}^{\infty}$ (the symbol $\frac{1}{4 \pi|\xi|^{2}}$ is not absolutely integrable near the origin of the frequency space $\mathbb{R}^{2} \times \mathbb{Z}$ ). However, the ambiguity is only up to constant functions, which will not be an issue since every appearance of $\Delta^{-1}$ will eventually be combined with at least one spatial derivative. For sake of concreteness, though, we fix an explicit choice ${ }^{18}$ of $\Delta^{-1} \omega$ for $\omega \in C_{c}^{\infty} \cap \Lambda_{0}\left(\mathbb{R}^{2} \times \mathbb{R} / \mathbb{Z}\right)$ by the formula

$$
\Delta^{-1} \omega(x)=\int_{\mathbb{R}^{2} \times \mathbb{R} / \mathbb{Z}} \omega\left(x^{\prime}\right) K_{1}\left(x-x^{\prime}\right) d \operatorname{vol}\left(x^{\prime}\right)
$$

\footnotetext{
18 In the language of distributions, this corresponds to fixing an explicit interpretation of the symbol $\frac{1}{4 \pi|\xi|^{2}}$ as a tempered distribution, which is well defined up to a constant multiple of the Dirac mass at the origin.
} 
where the fundamental solution $K_{1}(x)$ for $x \neq 0$ can be obtained via descent from the fundamental solution $\frac{1}{4 \pi|x|}$ on $\mathbb{R}^{3}$ by the renormalised summation formula

$$
K_{1}(x):=\lim _{N \rightarrow \infty} \sum_{n=-N}^{N} \frac{1}{4 \pi|\tilde{x}+(0,0, n)|}-\frac{\log N}{2 \pi}
$$

where $\tilde{x}$ is an arbitrary lift of $x$ from $\mathbb{R}^{2} \times \mathbb{R} / \mathbb{Z}$ to $\mathbb{R}^{3}$ (it is easy to see that the precise choice of lift is irrelevant). Roughly speaking, this kernel behaves like $\frac{1}{4 \pi|x|}$ when $|x|$ is small and like $\log |x|$ when $|x|$ is large. Note that the convergence of the sum in (8.4) improves after taking at least one derivative; for instance, one has the absolutely convergent series representation

$$
\nabla K_{1}(x)=\sum_{n=-\infty}^{\infty} \frac{-(\tilde{x}+(0,0, n))}{4 \pi|\tilde{x}+(0,0, n)|^{3}}
$$

Since the Hodge Laplacian $\Delta$ is diagonalised by the basis $d x^{1} \wedge d x^{2}, d x^{1} \wedge d x^{3}, d x^{2} \wedge$ $d x^{3}$, one could also write the last three terms in (8.3) more compactly as $\tilde{\eta}^{-1} \Delta^{-1} \omega$, as per (1.13). Observe that if $\tilde{A}_{0}$ is a pseudodifferential operator of order -1 , then $\tilde{A}$ will be a pseudodifferential operator of order -2 (formally, at least); similarly, if $A_{0}$ and $A_{0}^{*}$ commute with translations in the $x^{3}$ direction, then so does $\tilde{A}$.

From definition and integration by parts it is clear that $\tilde{A}$ is formally self-adjoint in the sense of (1.12). Next, we introduce the 2-form $\omega \in \Lambda_{2}\left(\mathbb{R}^{2} \times \mathbb{R} / \mathbb{Z}\right)$ and the vector field $\tilde{u} \in \Gamma^{1}\left(\mathbb{R}^{2} \times \mathbb{R} / \mathbb{Z}\right)$ at any given time by the formulae

$$
\begin{aligned}
\omega & :=d\left(\tilde{\theta} d x^{3}\right) \\
& =\left(\partial_{1} \tilde{\theta}\right) d x^{1} \wedge d x^{3}+\left(\partial_{2} \tilde{\theta}\right) d x^{2} \wedge d x^{3} \\
\tilde{u} & :=\tilde{u}^{1} \frac{d}{d x^{1}}+\tilde{u}^{2} \frac{d}{d x^{2}}-\tilde{\theta} \frac{d}{d x^{3}} .
\end{aligned}
$$

where $\tilde{\theta}:=\theta \circ \pi, \tilde{u}^{1}:=u^{1} \circ \pi, \tilde{u}^{2}:=u^{2} \circ \pi$ are the lifts of $\theta, u^{1}, u^{2}$ from $\mathbb{R}^{2}$ to $\mathbb{R}^{2} \times \mathbb{R} / \mathbb{Z}$. It is clear that $\omega$ is closed, and thus lies in $B^{2}\left(\mathbb{R}^{2} \times \mathbb{R} / \mathbb{Z}\right)$.

We now claim

Proposition 8.1 $\omega$ and $\tilde{u}$ (formally) obey the generalised Euler equations (1.10), (1.11) on $\mathbb{R}^{2} \times \mathbb{R} / \mathbb{Z}$ with vector potential operator $\tilde{A}$.

Proof We begin with (1.10). By (8.3), (8.6) we have

$$
\begin{aligned}
\tilde{A} \omega= & -\tilde{A}_{0} \Delta^{-1}\left(\partial_{1} \partial_{1} \tilde{\theta}+\partial_{2} \partial_{2} \tilde{\theta}\right) \frac{d}{d x^{1}} \wedge \frac{d}{d x^{2}} \\
& +\Delta^{-1} \partial_{1} \tilde{\theta} \frac{d}{d x^{1}} \wedge \frac{d}{d x^{3}} \\
& +\Delta^{-1} \partial_{2} \tilde{\theta} \frac{d}{d x^{2}} \wedge \frac{d}{d x^{3}} .
\end{aligned}
$$


But since $\tilde{\theta}=\theta \circ \pi$ is constant in the $x^{3}$ direction, we have from definition of the Hodge Laplacian that

$$
\partial_{1} \partial_{1} \theta+\partial_{2} \partial_{2} \tilde{\theta}=-\Delta \tilde{\theta}
$$

Taking divergences, and again noting that $\tilde{\theta}$ is constant in the $x^{3}$ direction, we have

$$
\begin{aligned}
\delta \tilde{A} \omega= & -\partial_{1} \tilde{A}_{0} \tilde{\theta} \frac{d}{d x^{2}}+\partial_{2} \tilde{A}_{0} \tilde{\theta} \frac{d}{d x^{1}} \\
& +\partial_{1} \Delta^{-1} \partial_{1} \tilde{\theta} \frac{d}{d x^{3}} \\
& +\partial_{2} \Delta^{-1} \partial_{2} \tilde{\theta} \frac{d}{d x^{3}} .
\end{aligned}
$$

From (6.2), (6.3), (8.1) one has

$$
\tilde{u}^{1}=\partial_{2} \tilde{A}_{0} \tilde{\theta} ; \quad \tilde{u}^{2}=-\partial_{1} \tilde{A}_{0} \tilde{\theta}
$$

inserting this and (8.8) into the above computation, we obtain (1.11).

Now we turn to (1.10). From (8.6) we have

$$
\omega=d \tilde{\theta} \wedge d x^{3}
$$

and hence by (1.7) and (2.8), we have

$$
\partial_{t} \omega+\mathcal{L}_{\tilde{u}} \omega=d\left(\partial_{t} \tilde{\theta}+\mathcal{L}_{\tilde{u}} \tilde{\theta}\right) \wedge d x^{3}-d \tilde{\theta} \wedge d\left(\mathcal{L}_{\tilde{u}} x^{3}\right) .
$$

From (8.7) we have

$$
\mathcal{L}_{\tilde{u}} x^{3}=-\tilde{\theta}
$$

and hence

$$
d \tilde{\theta} \wedge d\left(\mathcal{L}_{\tilde{u}} x^{3}\right)=-d \tilde{\theta} \wedge d \tilde{\theta}=0
$$

Next, since $\tilde{u}=u \circ \pi-\tilde{\theta} \frac{d}{d x^{3}}$ and $\tilde{\theta}$ is constant in the $x^{3}$ variable, we have

$$
\mathcal{L}_{\tilde{u}} \theta=\left(\mathcal{L}_{u} \theta\right) \circ \pi
$$

and hence by $(6.1)$

$$
\partial_{t} \tilde{\theta}+\mathcal{L}_{\tilde{u}} \tilde{\theta}=0
$$

The claim (1.10) follows. 


\subsection{Rigorous Construction}

We now prove Theorem 1.12 rigorously. Set $\mathcal{M}=\mathbb{R}^{2} \times \mathbb{R} / \mathbb{Z}$, and let $\varepsilon>0$. Let $M>1$ be sufficiently large depending on $\varepsilon$. Let $A_{0}: \Lambda_{0}\left(\mathbb{R}^{2}\right) \rightarrow \Lambda_{0}\left(\mathbb{R}^{2}\right)$ be the linear operator defined in (6.5), thus

$$
A_{0}(\theta)\left(x^{1}, x^{2}\right):=\frac{2}{M} \sum_{k=0}^{\infty} 2^{2 k} x^{1} \eta\left(2^{k} x^{2}\right) \psi\left(2^{k} x^{1}\right) \int_{\mathbb{R}^{2}} \theta(y) \varphi\left(2^{k} y\right) d \operatorname{vol}(y) .
$$

We then define the extension $\tilde{A}_{0}: \Lambda_{0}\left(\mathbb{R}^{2} \times \mathbb{R} / \mathbb{Z}\right) \rightarrow \Lambda_{0}\left(\mathbb{R}^{2} \times \mathbb{R} / \mathbb{Z}\right)$ by the formula

$$
\begin{aligned}
\tilde{A}_{0}(\theta)\left(x^{1}, x^{2}, x^{3}\right):= & \frac{2}{M} \sum_{k=0}^{\infty} 2^{2 k} x^{1} \eta\left(2^{k} x^{2}\right) \psi\left(2^{k} x^{1}\right) \int_{\mathbb{R}} \int_{\mathbb{R}^{2}} \theta\left(y, x^{3}\right. \\
& \left.+2^{-k} z\right) \varphi\left(2^{k} y\right) d \operatorname{vol}(y) \kappa(z) d z
\end{aligned}
$$

where $\kappa: \mathbb{R} \rightarrow \mathbb{R}$ is a smooth function supported on $[-1 / 2,1 / 2]$ with $\int_{\mathbb{R}} \kappa(z) d z=1$. The purpose of the additional averaging in the $z$ variable is so that $\tilde{A}_{0}$ obeys the kernel estimates (1.19) in the definition of a reasonable operator.

It is easy to see that the sum defining $\tilde{A}_{0}(\theta)$ is absolutely convergent for $\theta$ in $C_{c}^{\infty} \cap \Lambda_{0}\left(\mathbb{R}^{2} \times \mathbb{R} / \mathbb{Z}\right)$; indeed, the summands have size $O_{\theta}\left(2^{-j}\right)$. It is also easy to verify the relation (8.1) with $\theta \in C_{c}^{\infty} \cap \Lambda_{0}\left(\mathbb{R}^{2}\right)$. The adjoint map $\tilde{A}_{0}^{*}: \Lambda_{0}\left(\mathbb{R}^{2} \times \mathbb{R} / \mathbb{Z}\right) \rightarrow$ $\Lambda_{0}\left(\mathbb{R}^{2} \times \mathbb{R} / \mathbb{Z}\right)$ is given by the formula

$$
\begin{aligned}
\tilde{A}_{0}^{*}(\theta)\left(y^{1}, y^{2}, y^{3}\right):= & \frac{2}{M} \sum_{k=0}^{\infty} 2^{2 k} \varphi\left(2^{k} y^{1}, 2^{k} y^{2}\right) \int_{\mathbb{R}} \int_{\mathbb{R}^{2}} x^{1} \eta\left(2^{k} x^{2}\right) \psi\left(2^{k} x^{1}\right) \theta\left(x, y^{3}\right. \\
& \left.-2^{-k} z\right) d x \kappa(z) d z
\end{aligned}
$$

again, one can check that the sum defining $\tilde{A}_{0}^{*}$ is absolutely convergent for $\theta \in C_{c}^{\infty} \cap$ $\Lambda_{0}\left(\mathbb{R}^{2} \times \mathbb{R} / \mathbb{Z}\right)$, that $\tilde{A}_{0}^{*}$ is the adjoint of $\tilde{A}_{0}$ in the sense of (8.2), and that $\tilde{A}_{0}^{*}$ extends $A_{0}^{*}$. Finally it is clear from construction that $\tilde{A}_{0}$ and $\tilde{A}_{0}^{*}$ are both invariant with respect to translations in the $x^{3}$ direction.

Now we establish

Proposition 8.2 $\tilde{A}$ is a 100-reasonable vector potential operator.

Proof We first prove (1.20). As in the previous section, it will suffice to establish the slightly stronger bounds

$$
\|\tilde{A} \omega\|_{\dot{H}^{k+2}(\mathcal{M})} \lesssim\|\omega\|_{\dot{H}^{k}(\mathcal{M})}
$$

for all $0 \leqslant k \leqslant 100$.

The claim is clear for the last three components of (8.3), so we focus on the first three components. By duality (and commuting $\Delta^{-1}$ with $\partial_{1}, \partial_{2}$ ) it will suffice to show that 


$$
\left\|\tilde{A}_{0} \partial_{l} \omega\right\|_{\dot{H}^{s}\left(\mathbb{R}^{2} \times \mathbb{R} / \mathbb{Z}\right)} \lesssim\|\omega\|_{\dot{H}^{s}\left(\mathbb{R}^{2} \times \mathbb{R} / \mathbb{Z}\right)}
$$

for all $-102 \leqslant s \leqslant 102, l=1,2$, and $\omega \in \dot{H}^{s}(\mathcal{M})$ (dropping the requirement that $\omega$ be divergence-free). For future reference we note that we will in fact gain an extra factor of $1 / M$, and show that

$$
\left\|\tilde{A}_{0} \partial_{l} \omega\right\|_{\dot{H}^{s}\left(\mathbb{R}^{2} \times \mathbb{R} / \mathbb{Z}\right)} \lesssim \frac{1}{M}\|\omega\|_{\dot{H}^{s}\left(\mathbb{R}^{2} \times \mathbb{R} / \mathbb{Z}\right)} .
$$

Unwinding the definition of the Sobolev norms, it suffices to show that

$$
\left\|\Delta^{s / 2} \tilde{A}_{0} \partial_{l} \Delta^{-s / 2} f\right\|_{L^{2}\left(\mathbb{R}^{2} \times \mathbb{R} / \mathbb{Z}\right)} \lesssim \frac{1}{M}\|f\|_{L^{2}\left(\mathbb{R}^{2} \times \mathbb{R} / \mathbb{Z}\right)}
$$

for all $f \in L^{2}\left(\mathbb{R}^{2} \times \mathbb{R} / \mathbb{Z}\right)$. By Minkowski's inequality and translation invariance, it suffices to prove this with $\tilde{A}_{0}$ replaced by the variant operator $\tilde{A}_{0}^{\prime}$ defined by

$$
\tilde{A}_{0}^{\prime}(\theta)\left(x^{1}, x^{2}, x^{3}\right):=\frac{2}{M} \sum_{k=0}^{\infty} 2^{2 k} x^{1} \gamma\left(2^{k} x^{2}\right) \psi\left(2^{k} x^{1}\right) \int_{\mathbb{R}^{2}} \theta\left(y, x^{3}\right) \varphi\left(2^{k} y\right) d \operatorname{vol}(y)
$$

thus $\tilde{A}_{0}^{\prime}$ simply applies the operator $A_{0}$ on each $x^{3}$ slice of $\mathbb{R}^{2} \times \mathbb{R} / \mathbb{Z}$. Taking Fourier coefficients in the $\mathbb{R} / \mathbb{Z}$ coordinate (noting that $A_{0}^{\prime}$ and $\partial_{i}$ commute with this operation), it suffices to show the two-dimensional estimate

$$
\left\|(E+\Delta)^{s / 2} A_{0} \nabla(E+\Delta)^{-s / 2} f\right\|_{L^{2}\left(\mathbb{R}^{2}\right)} \lesssim \frac{1}{M}\|f\|_{L^{2}\left(\mathbb{R}^{2}\right)}
$$

for all $f \in L^{2}\left(\mathbb{R}^{2}\right)$ and $E \geqslant 0$, where $\Delta$ now denotes the Hodge Laplacian on $\mathbb{R}^{2}$ rather than $\mathbb{R}^{2} \times \mathbb{R} / \mathbb{Z}$.

Fix $E \geqslant 0$. By duality, it suffices to establish the bound

$$
\left|\left\langle A_{0} \nabla(E+\Delta)^{-s / 2} f,(E+\Delta)^{s / 2} g\right\rangle\right| \lesssim \frac{1}{M}\|f\|_{L^{2}\left(\mathbb{R}^{2}\right)}\|g\|_{L^{2}\left(\mathbb{R}^{2}\right)}
$$

for $f, g \in L^{2}\left(\mathbb{R}^{2}\right)$. By (6.5) and integration by parts, the left-hand side is $-\frac{1}{M} \sum_{k=0}^{\infty} X_{k} Y_{k}$, where

$$
X_{k}:=2^{k} \int_{\mathbb{R}^{2}}(E+\Delta)^{-s / 2} f(y)(\nabla \varphi)\left(2^{k} y\right) d \operatorname{vol}(y)
$$

and

$$
Y_{k}:=2^{k} \int_{\mathbb{R}^{2}}(E+\Delta)^{s / 2} g(x) 2^{k} x^{1} \gamma\left(2^{k} x^{2}\right) \psi\left(2^{k} x^{1}\right) d \operatorname{vol}(x) .
$$

The functions $y \mapsto \nabla \varphi(y)$ and $\left(x^{1}, x^{2}\right) \mapsto x^{1} \gamma\left(x^{2}\right) \psi\left(x^{1}\right)$ are smooth and compactly supported, and orthogonal to all polynomials of degree up to 1000, thus their Fourier 
transforms are Schwartz functions that vanish to order 1000 at the origin. From this, Plancherel's theorem, and Cauchy-Schwarz, we see that

$$
X_{k} \lesssim \sum_{N}\left(E+N^{2}\right)^{-s / 2} \min \left(N / 2^{k}, 2^{k} / N\right)^{1000}\left\|P_{N} f\right\|_{L^{2}\left(\mathbb{R}^{2}\right)}
$$

and

$$
Y_{k} \lesssim \sum_{M}\left(E+M^{2}\right)^{-s / 2} \min \left(M / 2^{k}, 2^{k} / M\right)^{1000}\left\|P_{M} g\right\|_{L^{2}\left(\mathbb{R}^{2}\right)}
$$

where $N, M$ range over the dyadic numbers $2^{n}, n \in \mathbb{Z}$, and $P_{N}$ denotes the Fourier projection to frequencies $N \leqslant|\xi| \leqslant 2 N$. Multiplying and summing in $k$ and using the hypothesis $|k| \leqslant 102$, we conclude that

$$
\sum_{k=0}^{\infty} X_{k} Y_{k} \lesssim \sum_{N} \sum_{M} \min (M / N, N / M)^{100}\left\|P_{N} f\right\|_{L^{2}\left(\mathbb{R}^{2}\right)}\left\|P_{M} g\right\|_{L^{2}\left(\mathbb{R}^{2}\right)}
$$

and the claim now follows from Schur's test and Plancherel's theorem.

Now we prove (1.19). We need to show that the integral kernel of $\tilde{A}$ obeys the bounds

$$
\left|\nabla_{x}^{i} \nabla_{y}^{j} K(x, y)\right| \lesssim \max \left(|x-y|^{-i-j-1},|x-y|^{-i-j}\right)
$$

for $0 \leqslant i, j \leqslant 100$ with $i+j \geqslant 1$. The contribution of the last three components of $\tilde{A}$ in (8.3) are acceptable after differentiating (8.4) as in (8.5) (note here that it is important that $i+j \geq 1$ ). It remains to control the kernel of the first three components. This kernel on $\mathbb{R}^{2} \times \mathbb{R} / \mathbb{Z}$ (and its derivatives) can be obtained by descent from the kernel of the corresponding operator on $\mathbb{R}^{3}$ (and its derivatives) by summing over cosets of $\{0\} \times\{0\} \times \mathbb{Z}$ as in (8.4), (8.5). Thus, if we let $\tilde{K}$ denote the kernel of the first three components of $\tilde{A}$ on $\mathbb{R}^{3}$, it will suffice to show that

$$
\left|\nabla_{x}^{i} \nabla_{y}^{j} \tilde{K}(x, y)\right| \lesssim_{i, j}|x-y|^{-i-j-1}
$$

for $0 \leqslant i, j \leqslant 100$ with $i+j \geqslant 1$; the condition $i+j \geq 1$ is needed to ensure a convergent sum over the coset of $\{0\} \times\{0\} \times \mathbb{Z}$, but will not otherwise be needed henceforth.

By linearity and taking adjoints, it thus suffices to verify the above bound for the integral kernel of $\tilde{A}_{0} \Delta^{-1} \partial_{l}$ on $\mathbb{R}^{3}$ for $l=1,2$.

From the Newton formula

$$
\Delta^{-1} f(w)=\frac{1}{4 \pi} \int_{\mathbb{R}^{3}} \frac{f(y)}{|w-y|} d \operatorname{vol}(y)
$$


on $\mathbb{R}^{3}$, we see that the kernel $\Delta^{-1} \partial_{l}$ is given by $L(w-y)$, where

$$
L(x):=\frac{-1}{4 \pi} \frac{x^{l}}{|x|^{3}}
$$

also, from (8.9), the kernel $R(x, w)$ of $\tilde{A}_{0}$ is given by

$$
R(x, w)=\sum_{k=0}^{\infty} R_{k}(x, w)
$$

where

$$
R_{k}(x, w):=\frac{2}{M} 2^{3 k} x^{1} \gamma\left(2^{k} x^{2}\right) \psi\left(2^{k} x^{1}\right) \varphi\left(2^{k}\left(w^{1}, w^{2}\right)\right) \kappa\left(2^{k}\left(x^{3}-w^{3}\right)\right) .
$$

Thus it will suffice to show that

$$
\left|\sum_{k=0}^{\infty} \nabla_{x}^{i} \nabla_{y}^{j} \int_{\mathbb{R}^{3}} R_{k}(x, w) L(w-y) d \operatorname{vol}(w)\right| \lesssim|x-y|^{-i-j-1}
$$

for $0 \leqslant i, j \leqslant 100$.

From the construction of $\gamma, \psi, \varphi, \kappa$ we see that $R_{k}(x, w)$ is supported on the region $|x-w| \leqslant 100 \times 2^{-k}$ and obeys the derivative bounds

$$
\left|\nabla_{x}^{i} \nabla_{w}^{j} R_{k}(x, w)\right| \lesssim 2^{(i+j+2) k}
$$

for $0 \leqslant i, j \leqslant 100$. Also, from the moment conditions on $\varphi$ we see that for any $x \in \mathbb{R}^{3}$, the function $w \mapsto R_{k}(x, w)$ is orthogonal to any polynomial of degree at most 1000 .

Let us first consider the contribution to the left-hand side of (8.12) of those $k$ for which

$$
|x-y| \geqslant 200 \times 2^{-k} .
$$

Then we have $|w-y| \gtrsim|x-y|$, and hence $\left|\nabla_{y}^{m} L(w-y)\right| \lesssim|x-y|^{-2-m}$ for any $0 \leqslant m \leqslant 1000$. For each fixed $x \in \mathbb{R}^{3}$, and for $w$ in the support of $R_{k}(x, w)$, one can then use Taylor expansion to write $\nabla^{j} L(w-y)$ as a polynomial of degree at most 1000 , plus an error of size at most $O\left(\left(2^{-k} /|x-y|\right)^{500}|x-y|^{-2-j}\right)$ (say). Using (8.13) (with $j$ replaced by 0 ), and the support of $R_{k}$, we conclude that

$$
\left|\nabla_{x}^{i} \nabla_{y}^{j} \int_{\mathbb{R}^{3}} R_{k}(x, w) L(w-y) d w\right| \lesssim 2^{(i+2) k} \times 2^{-3 k} \times\left(2^{-k} /|x-y|\right)^{500}|x-y|^{-2-j} .
$$

Summing over all $k$ obeying (8.14), we see that this contribution to the left-hand side of (8.12) is acceptable. 
It remains to treat the contribution of those $k$ for which (8.14) fails. In this case we integrate by parts to obtain the identity

$$
\left|\nabla_{x}^{i} \nabla_{y}^{j} \int_{\mathbb{R}^{3}} R_{k}(x, w) L(w-y) d \operatorname{vol}(w)\right|=\left|\int_{\mathbb{R}^{3}} \nabla_{x}^{i} \nabla_{w}^{j} R_{k}(x, w) L(w-y) d \operatorname{vol}(w)\right| .
$$

Applying (8.13) and the support of $R_{k}$, we conclude that

$$
\left|\nabla_{x}^{i} \nabla_{y}^{j} \int_{\mathbb{R}^{3}} R_{k}(x, w) L(w-y) d \operatorname{vol}(w)\right| \lesssim 2^{(i+j+2) k} \int_{|w-x| \leqslant 100 \times 2^{-k}}|L(w-y)| d \operatorname{vol}(w) .
$$

Since (8.14) fails, the condition $|w-x| \leqslant 100 \times 2^{-k}$ implies that $|w-y| \lesssim 2^{-k}$, and hence by the bound $|L(w-y)| \lesssim|w-y|^{-2}$, we have

$$
2^{(i+j+2) k} \int_{|w-x| \leqslant 100 \times 2^{-k}}|L(w-y)| d \operatorname{vol}(w) \lesssim 2^{(i+j+1) k} .
$$

Summing over all $k$ for which (8.14) fails, we see that this contribution to (8.12) is also acceptable.

Next, we establish positive definiteness.

Proposition 8.3 For any $\omega \in C_{c}^{\infty} \cap B_{2}(\mathcal{M})$, we hqave

$$
\int_{\mathcal{M}}\langle\omega, \tilde{A} \omega\rangle d \mathrm{vol}=\left(1+O\left(\frac{1}{M}\right)\right)\|\omega\|_{\dot{H}^{-1}(\mathcal{M})}^{2} .
$$

Proof From Plancherel's theorem, the contribution of the last three terms of (8.3) to the left-hand side of (8.15) is precisely $\|\omega\|_{\dot{H}^{-1}(\mathcal{M})}^{2}$. By the Cauchy-Schwarz inequality and the triangle inequality, it thus suffices to establish the bounds

$$
\left\|\tilde{A}_{0} u\right\|_{\dot{H}^{1}(\mathcal{M})} \lesssim \frac{1}{M}\|u\|_{L^{2}(\mathcal{M})}
$$

and

$$
\left\|\tilde{A}_{0}^{*} v\right\|_{L^{2}(\mathcal{M})} \lesssim \frac{1}{M}\|v\|_{\dot{H}^{-1}(\mathcal{M})}
$$

for $u \in L^{2}(\mathcal{M})$ and $v \in \dot{H}^{-1}(\mathcal{M})$. But this follows from (8.11) and duality.

Let $\theta_{0}: \mathbb{R}^{2} \rightarrow \mathbb{R}$ be initial data of the type in Proposition 6.1, and let $\tilde{\theta}_{0}: \mathcal{M} \rightarrow \mathbb{R}$ be the lift of $\theta_{0}$ to $\mathcal{M}$ defined by $\tilde{\theta}_{0}:=\theta_{0} \circ \pi$. Following (8.6), we define the initial data $\omega_{0} \in C_{c}^{\infty} \cap B_{2}(\mathcal{M})$ by the formula

$$
\omega_{0}:=\left(\partial_{1} \tilde{\theta}_{0}\right) d x^{1} \wedge d x^{3}+\left(\partial_{2} \tilde{\theta}_{0}\right) d x^{2} \wedge d x^{3} .
$$

We now claim (for $M$ sufficiently large) that Theorem 1.12 holds with this choice of initial data $\omega_{0}$ and with the operator $\tilde{A}$ constructed above as vector potential operator. 
We have already verified that $\tilde{A}$ is 100 -reasonable, formally self-adjoint, and obeys (1.21) (if $M$ is sufficiently large depending on $\varepsilon$ ). Thus, the only way that Theorem 1.12 can still fail is if there is a solution $\omega \in X^{10,2}, u \in Y^{10,2}$ to the generalised Euler equations with vector potential operator $\tilde{A}$ and initial vorticity $\omega_{0}$ on the time interval $[0,1]$.

Suppose for contradiction that this is the case. Obseve that $\omega_{0}$ is invariant with respect to translations in the $x^{3}$ direction, and that $\tilde{A}$ commutes with these translations. Thus, if $\omega, u$ solve the generalised Euler equations with initial data $\omega_{0}$, then so do any translates of $\omega, u$ in the $x^{3}$ direction. Applying the uniqueness component of Theorem 1.9 , we conclude that $\omega, u$ are invariant with respect to translations in the $x^{3}$ direction, thus

$$
\partial_{3} \omega=0 ; \quad \partial_{3} u=0
$$

We define the scalar field $\tilde{\theta}:[0,1] \times \mathcal{M} \rightarrow \mathbb{R}$ by solving the transport equation

$$
\partial_{t} \tilde{\theta}+\mathcal{L}_{u} \tilde{\theta}=0
$$

with initial data $\tilde{\theta}=\tilde{\theta}_{0}$. Since $u$ lies in $Y^{10,2}$ and $\tilde{\theta}_{0}$ is smooth and compactly supported, there is no difficulty defining $\tilde{\theta}$ uniquely, in such a way that it is continuously differentiable in both space and time, and compactly supported in space. Since $\tilde{\theta}$ and $u$ are invariant with respect to translations in the $x^{3}$ direction, $\tilde{\theta}$ is also.

We now can justify the formal ansatz (8.6):

Proposition 8.4 On $[0,1] \times \mathcal{M}$, we have

$$
\begin{aligned}
\omega & =d \tilde{\theta} \wedge d x^{3} \\
& =\partial_{1} \tilde{\theta} d x^{1} \wedge d x^{3}+\partial_{2} \tilde{\theta} d x^{2} \wedge d x^{3} .
\end{aligned}
$$

Proof Set $\alpha$ to be the 2-form

$$
\alpha:=\omega-d \tilde{\theta} \wedge d x^{3}
$$

then $\alpha$ is continuously differentiable in space and time, and our task is to show that $\alpha(t)=0$ for all $t \in[0,1]$. From (8.16) we know that $\alpha(0)=0$. We now use (1.10), (8.17) to compute

$$
\begin{aligned}
\left(\partial_{t}+\mathcal{L}_{u}\right) \alpha & =\left(\partial_{t}+\mathcal{L}_{u}\right) \alpha-\left(\partial_{t}+\mathcal{L}_{u}\right)\left(d \tilde{\theta} \wedge d x^{3}\right) \\
& =0-d 0 \wedge d x^{3}-d \tilde{\theta} \wedge d \mathcal{L}_{u} x^{3} \\
& =-d \tilde{\theta} \wedge d u^{3}
\end{aligned}
$$

On the other hand, from (1.11) and (8.3) we have

$$
\begin{aligned}
u^{3}= & \partial_{1} \partial_{1} \Delta^{-1} \tilde{A}_{0}^{*} \omega_{12}+\partial_{2} \partial_{2} \Delta^{-1} \tilde{A}_{0}^{*} \omega_{12} \\
& +\partial_{1} \Delta^{-1} \omega_{13}+\partial_{2} \Delta^{-1} \omega_{23}
\end{aligned}
$$




$$
\begin{aligned}
= & \tilde{A}_{0}^{*} \alpha_{12}+\partial_{1} \Delta^{-1} \partial_{1} \tilde{\theta}+\partial_{2} \Delta^{-1} \partial_{2} \tilde{\theta} \\
& +\partial_{1} \Delta^{-1} \alpha_{13}+\partial_{2} \Delta^{-1} \alpha_{23} \\
= & \left(\tilde{A}_{0}^{*} \alpha_{12}+\partial_{1} \Delta^{-1} \alpha_{13}+\partial_{2} \Delta^{-1} \alpha_{23}\right)-\tilde{\theta} .
\end{aligned}
$$

Since $d \tilde{\theta} \wedge d \tilde{\theta}=0$, we thus have

$$
\left(\partial_{t}+\mathcal{L}_{u}\right) \alpha=-d \tilde{\theta} \wedge d\left(\tilde{A}_{0}^{*} \alpha_{12}+\partial_{1} \Delta^{-1} \alpha_{13}+\partial_{2} \Delta^{-1} \alpha_{23}\right) .
$$

Taking inner products with $\alpha$ and integrating by parts (which can be justified as $\omega$ lies in $X^{10,2}$ and $\theta$ is continuously differentiable and compactly supported), we conclude that

$$
\partial_{t}\|\alpha\|_{L^{2}}^{2}=-2\left\langle d \tilde{\theta} \wedge d\left(\tilde{A}_{0}^{*} \alpha_{12}+\partial_{1} \Delta^{-1} \alpha_{13}+\partial_{2} \Delta^{-1} \alpha_{23}\right), \alpha\right\rangle_{L^{2}(\mathcal{M})} .
$$

From the proof of Proposition 8.2, we know that $\tilde{A}_{0}^{*}$ maps $L^{2}(\mathcal{M})$ to $\dot{H}^{1}(\mathcal{M})$. As $d \tilde{\theta}$ is bounded, we conclude that

$$
\partial_{t}\|\alpha\|_{L^{2}}^{2} \lesssim \theta\|\alpha\|_{L^{2}}^{2}
$$

and hence from Gronwall's inequality we have $\alpha(t)=0$ for all $0 \leqslant t \leqslant 1$, as required.

If we insert the above proposition back into (8.3), we have

$$
\begin{aligned}
\tilde{A} \omega:= & -\tilde{A}_{0} \Delta^{-1}\left(\partial_{1} \partial_{1} \tilde{\theta}+\partial_{2} \partial_{2} \tilde{\theta}\right) \frac{d}{d x^{1}} \wedge \frac{d}{d x^{2}} \\
& +\Delta^{-1} \partial_{1} \tilde{\theta} \frac{d}{d x^{1}} \wedge \frac{d}{d x^{3}} \\
& +\Delta^{-1} \partial_{2} \tilde{\theta} \frac{d}{d x^{2}} \wedge \frac{d}{d x^{3}} .
\end{aligned}
$$

The first term on the right-hand side simplifies to $\tilde{A}_{0} \tilde{\theta} \frac{d}{d x^{1}} \wedge \frac{d}{d x^{2}}$. Taking divergences (and recalling that $\tilde{\theta}$ is constant in the $x^{3}$ direction), and using (1.11), we conclude that

$$
u=\partial_{2}\left(\tilde{A}_{0} \tilde{\theta}\right) \frac{d}{d x^{1}}-\partial_{1}\left(\tilde{A}_{0} \tilde{\theta}\right) \frac{d}{d x^{2}}-\tilde{\theta} \frac{d}{d x^{3}}
$$

(cf. (8.7)). The equation (8.17) then becomes

$$
\partial_{t} \tilde{\theta}+\partial_{2}\left(\tilde{A}_{0} \tilde{\theta}\right) \partial_{1} \tilde{\theta}-\partial_{1}\left(\tilde{A}_{0} \tilde{\theta}\right) \partial_{2} \tilde{\theta}=0 .
$$

Since $\tilde{\theta}$ is constant in the $x^{3}$ direction, we can write $\tilde{\theta}=\theta \circ \pi$ for some continuously differentiable, compactly supported $\theta:[0,1] \times \mathbb{R}^{2} \rightarrow \mathbb{R}$. From (8.1) we then have

$$
\partial_{t} \theta+\partial_{2}\left(A_{0} \theta\right) \partial_{1} \theta-\partial_{1}\left(A_{0} \theta\right) \partial_{2} \theta=0 .
$$


But then $\theta$ contradicts Proposition 6.1 (with $u^{1}:=\partial_{2}\left(A_{0} \theta\right)$ and $u^{1}:=-\partial_{1}\left(A_{0} \theta\right)$ ), as required.

Remark 8.5 Applying the above arguments with $A_{0}$ replaced by the SQG vector potential operator $\Delta^{-1 / 2}$, we obtain a rigorous connection between SQG and an explicit three dimensional generalised Euler equation. Namely, if there exists a finite time blowup solution to SQG in $\mathbb{R}^{2}$ (with suitable decay at infinity), then there exists a finite time blowup solution to a generalised Euler equation in $\mathbb{R}^{2} \times \mathbb{R} / \mathbb{Z}$ for an explicit vector potential operator $A$ that is a Fourier multiplier of order -2 which is self-adjoint and positive definite.

\section{Removing the Periodicity}

We now modify the arguments of the previous section to prove Theorem 1.13. Let $\mathcal{M}$ denote the Euclidean manifold that is represented in Cartesian coordinates by $\mathbb{R}^{3}$. Whereas in previous sections we would use the notations $\mathcal{M}$ and $\mathbb{R}^{3}$ interchangeably, in this section we will take care to distinguish the manifold $\mathcal{M}$ from its Cartesian coordinate representation $\mathbb{R}^{3}$. This is because we will be using a number of other coordinate systems for $\mathcal{M}$, such as cylindrical coordinates, in which the coordinate space is not $\mathbb{R}^{3}$. More precisely, for any triple $\left(x^{1}, x^{2}, x^{3}\right) \in \mathbb{R}^{3}$ of real numbers, we let $\left(x^{1}, x^{2}, x^{3}\right)_{\text {car }} \in \mathcal{M}$ denote the associated point on $\mathcal{M}$, thus the map $\left(x^{1}, x^{2}, x^{3}\right) \mapsto$ $\left(x^{1}, x^{2}, x^{3}\right)_{\text {car }}$ gives an isomorphism between $\mathbb{R}^{3}$ and $\mathcal{M}$; however we will not view this isomorphisms as an identification, keeping the point $\left(x^{1}, x^{2}, x^{3}\right)_{\text {car }} \in \mathcal{M}$ and the triple $\left(x^{1}, x^{2}, x^{3}\right) \in \mathbb{R}^{3}$ conceptually distinct.

As mentioned in the introduction, the strategy is to try to embed $\mathbb{R}^{2} \times \mathbb{R} / \mathbb{Z}$ (or more precisely, $\mathbb{R}^{2} \times \mathbb{R} / \mathbb{Z}$ equipped with a constant coefficient Riemannian metric) into $\mathcal{M}$. Clearly this cannot be done globally, and certainly not isometrically; however, it can be done locally, and nearly isometrically, by modifying the familiar cylindrical coordinates $^{19}(z, r, \alpha)_{\text {cyl }}$ of $\mathcal{M}$, with $(z, r, \alpha) \in \mathbb{R} \times[0,+\infty) \times \mathbb{R} / 2 \pi \mathbb{Z}$, defined in terms of the Cartesian coordinate system $\left(x^{1}, x^{2}, x^{3}\right)_{\text {car }}$ by the change of variables

$$
\left(x^{1}, x^{2}, x^{3}\right)_{\mathrm{car}}=(r \cos \alpha, r \sin \alpha, z)_{\mathrm{car}}=(z, r, \alpha)_{\mathrm{cyl}} .
$$

Of course, the cylindrical coordinate system is singular at the $x^{3}$-axis

$$
\left\{\left(x^{1}, x^{2}, x^{3}\right)_{\mathrm{car}}: x^{1}=x^{2}=0\right\}=\left\{(z, r, \alpha)_{\mathrm{cyl}}: r=0\right\},
$$

but let us ignore this singularity for the moment and work away from this axis, in which the map $(z, r, \alpha) \mapsto(z, r, \alpha)$ cyl becomes a diffeomorphism between (most of) $\mathbb{R} \times[0,+\infty) \times \mathbb{R} / 2 \pi \mathbb{Z}$ and (most of) $\mathcal{M}$. In cylindrical coordinates, the Euclidean first fundamental form

$$
d \eta^{2}=\left(d x^{1}\right)^{2}+\left(d x^{2}\right)^{2}+\left(d x^{3}\right)^{2}
$$

\footnotetext{
19 We use $\alpha$ here instead of $\theta$ to denote the angular variable, as we will reserve the latter symbol for an active scalar field later in this section.
} 
becomes

$$
d \eta^{2}=d z^{2}+d r^{2}+r^{2} d \alpha^{2}
$$

while the volume form

$$
d \mathrm{vol}=d x^{1} \wedge d x^{2} \wedge d x^{3}
$$

becomes

$$
d \mathrm{vol}=r d z \wedge d r \wedge d \alpha
$$

Note that the first fundamental form and the volume element both have variable coefficients due to the factors of $r$. In the latter case, we can rectify this by replacing the radial variable $r$ with the modified radial variable $y:=r^{2} / 2$, thus introducing ${ }^{20}$ a modified cylindrical coordinate system $(z, y, \alpha)_{\bmod }$ with $(z, y, \alpha) \in \mathbb{R} \times[0,+\infty) \times \mathbb{R} / 2 \pi \mathbb{Z}$, defined through the change of variables

$$
\left(x^{1}, x^{2}, x^{3}\right)_{\mathrm{car}}=(\sqrt{2 y} \cos \alpha, \sqrt{2 y} \sin \alpha, z)_{\mathrm{car}}=(z, \sqrt{2 y}, \alpha)_{\mathrm{cyl}}=(z, y, \alpha)_{\bmod } .
$$

The volume form is now constant coefficient,

$$
d \mathrm{vol}=d z \wedge d y \wedge d \theta
$$

so in particular the Hodge star $*$ and codifferential $\delta$ look the same when written in $(z, y, \alpha)_{\text {mod }}$ coefficients as they do in $\left(x^{1}, x^{2}, x^{3}\right)_{\text {car }}$ coordinates. However the first fundamental form remains variable coefficient:

$$
d \eta^{2}=d z^{2}+\frac{1}{2 y} d y^{2}+2 y d \alpha^{2} .
$$

Nevertheless, we observe that the first fundamental form is approximately constant coefficient when $y$ is large. Indeed, let $\varepsilon>0$ be the quantity in Theorem 1.13. If $M \geqslant 10^{10}$ is a large constant depending on $\varepsilon$ to be chosen later, and we reparameterise the annular region

$$
\left\{(z, y, \alpha)_{\bmod }:\left|y-M^{2} / 2\right|<M^{3 / 2} ;|z|<M^{1 / 2}\right\}
$$

in $\mathcal{M}$ using rescaled coordinates $\left(w^{1}, w^{2}, w^{3}\right)_{\text {rsc }}$, with $\left(w^{1}, w^{2}, w^{3}\right)$ confined to the region

$$
Q:=\left(-M^{1 / 2}, M^{1 / 2}\right) \times\left(-M^{1 / 2}, M^{1 / 2}\right) \times \mathbb{R} / 2 \pi M \mathbb{Z}
$$

\footnotetext{
20 This modified cylindrical coordinate system has been used previously to simplify the true Euler equations in the case of axisymmetric solutions with swirl; see [4,69].
} 
defined by

$$
(z, y, \alpha)_{\bmod }=\left(w^{1}, M^{2} / 2+M w^{2}, \frac{w^{3}}{M}\right)_{\bmod }=\left(w^{1}, w^{2}, w^{3}\right)_{\mathrm{rsc}}
$$

or equivalently

$$
\left(w^{1}, w^{2}, w^{3}\right)_{\mathrm{rsc}}=\left(\sqrt{M^{2} / 2+M w^{2}} \cos \frac{w^{3}}{M}, \sqrt{M^{2} / 2+M w^{2}} \sin \frac{w^{3}}{M}, w^{1}\right)_{\mathrm{car}}
$$

then the volume form is still constant coefficient in this region,

$$
d \text { vol }=d w^{1} \wedge d w^{2} \wedge d w^{3}
$$

and the first fundamental form is almost Euclidean:

$$
d \eta^{2}=\left(d w^{1}\right)^{2}+\left(1+\frac{2 w^{2}}{M}\right)^{-1}\left(d w^{2}\right)^{2}+\left(1+\frac{2 w^{2}}{M}\right)\left(d w^{3}\right)^{2}
$$

From this it is easy to see that the map $w \mapsto w_{\text {rsc }}$ is a bilipschitz identification of $Q$ (with the Euclidean metric) with the region (9.2), where the bilipschitz constants are bounded uniformly in $M$. It will later be convenient (mostly for notational reasons) to embed $Q$ as a subset of $\mathbb{R}^{2} \times \mathbb{R} / 2 \pi M \mathbb{Z}$, but we do not attempt to identify the remaining portion of $\mathbb{R}^{2} \times \mathbb{R} / 2 \pi M \mathbb{Z}$ with any portion of $\mathcal{M}$, thus leaving the $\left(w^{1}, w^{2}, w^{3}\right)$ coordinate system as a local coordinate system parameterising (9.2) only.

In order to smoothly interpolate between the Euclidean structure on $\mathbb{R}^{2} \times \mathbb{R} / 2 \pi M \mathbb{Z}$ and the Euclidean structure on $\mathbb{R}^{3}$, we will (for technical reasons) need a very gentle cutoff function $\varphi \in C^{c}(\mathcal{M})$ supported in (9.2) which is bounded by 1 and small in $\dot{H}^{1}(\mathcal{M})$, while remaining invariant with respect to rotations around the axis (9.1); this is possible due to the failure of the two-dimensional Sobolev embedding $\dot{H}^{1} \not \subset L^{\infty}$. More precisely, we set

$$
\varphi\left(\left(w^{1}, w^{2}, w^{3}\right)_{\mathrm{rsc}}\right):=h\left(w_{1}, w_{2}\right)
$$

in (9.2), with $\varphi$ vanishing outside of (9.2), where $h: \mathbb{R}^{2} \rightarrow[0,1]$ is a smooth, spherically symmetric function supported on $B_{\mathbb{R}^{2}}(0, \sqrt{M})$ which equals 1 on $B_{\mathbb{R}^{2}}\left(0,10^{3}\right)$, and is such that

$$
h(w)=1-\frac{\log |w|}{\log \sqrt{M}}
$$

when $10^{4} \leqslant|w| \leqslant \sqrt{M} / 10$, with the derivative estimates

$$
\left|\nabla^{j} h(w)\right| \lesssim j \frac{1}{\log M} \frac{1}{(1+|w|)^{j}}
$$

for all $j \geqslant 1$ and $w \in \mathbb{R}^{2}$. 
Let $I: \Lambda_{0}(\mathcal{M}) \rightarrow \Lambda_{0}\left(\mathbb{R}^{2} \times \mathbb{R} / 2 \pi M \mathbb{Z}\right)$ be the operator defined by

$$
\operatorname{If}\left(w^{1}, w^{2}, w^{3}\right):=(\varphi f)\left(\left(w^{1}, w^{2}, w^{3}\right)_{\mathrm{rsc}}\right)
$$

for $\left(w^{1}, w^{2}, w^{3}\right)$ in $Q$, with If vanishing outside of this region. The adjoint operator $I^{*}: \Lambda_{0}\left(\mathbb{R}^{2} \times \mathbb{R} / 2 \pi M \mathbb{Z}\right) \rightarrow \Lambda_{0}(\mathcal{M})$ is then given by the formula

$$
I^{*} f\left(\left(w^{1}, w^{2}, w^{3}\right)_{\mathrm{rsc}}\right)=\varphi\left(\left(w^{1}, w^{2}, w^{3}\right)_{\mathrm{rsc}}\right) f\left(w^{1}, w^{2}, w^{3}\right)
$$

in the annulus (9.2), with $I^{*} f$ vanishing outside of this annulus. (The fact that $I^{*}$ is the adjoint of $I$ follows from the fact that the volume form on $\mathcal{M}$ is given by $d w^{1} \wedge d w^{2} \wedge d w^{3}$ in (9.2), so there is no Jacobian factor.)

Let $\tilde{A}_{0}: \Lambda_{0}\left(\mathbb{R}^{2} \times \mathbb{R} / 2 \pi M \mathbb{Z}\right) \rightarrow \Lambda_{0}\left(\mathbb{R}^{2} \times \mathbb{R} / 2 \pi M \mathbb{Z}\right)$ be the operator defined by (8.9) (but now with the $x^{3}$ variable ranging in $\mathbb{R} / 2 \pi M \mathbb{Z}$ rather than $\mathbb{R} / \mathbb{Z}$ ). We now define the operator $\tilde{A}: B_{2}(\mathcal{M}) \rightarrow \Gamma^{2}(\mathcal{M})$ by the formula

$$
\tilde{A}=I^{*} A^{\prime} I+\tilde{\eta}^{-1} \Delta^{-1}-\varphi \tilde{\eta}^{-1} \Delta^{-1} \varphi
$$

where the operator $A^{\prime}: \Lambda_{2}\left(\mathbb{R}^{2} \times \mathbb{R} / 2 \pi M \mathbb{Z}\right) \rightarrow \Gamma^{2}\left(\mathbb{R}^{2} \times \mathbb{R} / 2 \pi M \mathbb{Z}\right)$ is given by the formula

$$
\begin{aligned}
A^{\prime} \omega:= & -\tilde{A}_{0} \Delta_{w}^{-1}\left(\partial_{1} \omega_{13}+\partial_{2} \omega_{23}\right) \frac{d}{d w^{1}} \wedge \frac{d}{d w^{2}} \\
& +\partial_{1} \Delta_{w}^{-1} \tilde{A}_{0}^{*} \omega_{12} \frac{d}{d w^{1}} \wedge \frac{d}{d w^{3}} \\
& +\partial_{2} \Delta_{w}^{-1} \tilde{A}_{0}^{*} \omega_{12} \frac{d}{d w^{2}} \wedge \frac{d}{d w^{3}} \\
& +\Delta_{w}^{-1} \omega_{13} \frac{d}{d w^{1}} \wedge \frac{d}{d w^{3}} \\
& +\Delta_{w}^{-1} \omega_{23} \frac{d}{d w^{2}} \wedge \frac{d}{d w^{3}} \\
& +\Delta_{w}^{-1} \omega_{12} \frac{d}{d w^{1}} \wedge \frac{d}{d w^{2}}
\end{aligned}
$$

where $\omega \in \Lambda_{2}\left(\mathbb{R}^{2} \times \mathbb{R} / 2 \pi M \mathbb{Z}\right)$ is expressed in coordinates as

$$
\omega=\omega_{12} d w^{1} \wedge d w^{2}+\omega_{13} d w^{1} \wedge d w^{3}+\omega_{23} d w^{2} \wedge d w^{3}
$$

and $\Delta_{w}$ denotes the Euclidean Laplacian on $\mathbb{R}^{2} \times \mathbb{R} / 2 \pi M \mathbb{Z}$ (the reader should take care to not confuse this with the Laplacian $\Delta$ on $\mathcal{M}$, although the two operators become close to each other in some sense when $M$ is large). As in the previous section, we need to fix an inverse of $\Delta_{w}^{-1}$; for sake of concreteness we set

$$
\Delta_{w}^{-1} \omega(w)=\int_{\mathbb{R}^{2} \times \mathbb{R} / 2 \pi M \mathbb{Z}} \omega\left(w^{\prime}\right) K_{2 \pi M}\left(w-w^{\prime}\right) d \operatorname{vol}\left(w^{\prime}\right)
$$


where

$$
K_{2 \pi M}(w):=\lim _{N \rightarrow \infty} \sum_{n=-N}^{N} \frac{1}{4 \pi|\tilde{w}+(0,0,2 \pi M n)|}-\frac{\log N}{2 \pi}
$$

and $\tilde{w}$ is an arbitrary lift of $w$ from $\mathbb{R}^{2} \times \mathbb{R} / 2 \pi M \mathbb{Z}$ to $\mathbb{R}^{3}$.

Informally, $\tilde{A}$ behaves like the true Euler vector potential $\tilde{\eta}^{-1} \Delta^{-1}$ away from (9.2), but inside the smaller region

$$
\left\{\left(w^{1}, w^{2}, w^{3}\right)_{\mathrm{rsc}}:\left(w^{1}, w^{2}\right) \in B_{\mathbb{R}^{2}}\left(0,10^{3}\right)\right\}
$$

it behaves (in $\left(w^{1}, w^{2}, w^{3}\right)$ coordinates) like the operator defined in (8.3).

It is easy to see that $\tilde{A}$ is well defined on $C_{c}^{\infty} \cap B_{2}(\mathcal{M})$ and formally self-adjoint. Now we verify the further properties of $\tilde{A}$ needed for Theorem 1.13.

Proposition 9.1 $\tilde{A}$ is a 100-reasonable vector potential operator.

Proof We begin with establishing (1.20). Let $0 \leqslant k \leqslant 100$. From standard elliptic estimates we see that the contribution of the $\tilde{\eta}^{-1} \Delta^{-1}$ term in (9.4) is acceptable. Now we turn to the $\varphi \tilde{\eta}^{-1} \Delta^{-1} \varphi$ term. If $\omega$ is bounded in $H^{k}(\mathcal{M})$, then from the Leibniz rule and Hölder's inequality $\varphi \omega$ is bounded in both $H^{k}(\mathcal{M})$ and $L^{1}(\mathcal{M})$. From Sobolev embedding we see that $\Delta^{-1} \varphi \omega$ is locally in $H^{k+2}(\mathcal{M})$ (this can be seen for instance by breaking up $\varphi \omega$ into low frequency and high frequency components), with bounds that are allowed to depend on $M$. From this and the Leibniz rule we see that $\varphi \tilde{\eta}^{-1} \Delta^{-1} \varphi \omega$ is bounded in $H^{k+2}(\mathcal{M})$, and from this we see that the contribution of the $\varphi \tilde{\eta}^{-1} \Delta^{-1} \varphi$ is also acceptable.

To finish the proof of (1.20), it will suffice to show that

$$
\left\|I^{*} A^{\prime} I \omega\right\|_{H^{k+2}(\mathcal{M})} \lesssim M\|\omega\|_{H^{k}(\mathcal{M})} .
$$

Changing variables to $\left(w^{1}, w^{2}, w^{3}\right)_{\text {rsc }}$ coordinates, we see that it suffices to show that

$$
\left\|\varphi A^{\prime} \omega\right\|_{H^{k+2}\left(\mathbb{R}^{2} \times \mathbb{R} / 2 \pi M \mathbb{Z}\right)} \lesssim M\|\omega\|_{H^{k}\left(\mathbb{R}^{2} \times \mathbb{R} / 2 \pi M \mathbb{Z}\right)}
$$

whenever $\omega$ is supported on the support of $\varphi$ (which by abuse of notation we now view as a function on $\mathbb{R}^{2} \times \mathbb{R} / 2 \pi M \mathbb{Z}$ ). The contribution of the $\Delta_{w}^{-1} \omega_{i j}$ terms in (9.5) for $i j=13,23,12$ can be treated by the same argument used to control $\varphi \tilde{\eta}^{-1} \Delta^{-1} \varphi$. It thus remains to show that

$$
\left\|\varphi \tilde{A}_{0} \Delta_{w}^{-1} \nabla \omega\right\|_{H^{k+2}\left(\mathbb{R}^{2} \times \mathbb{R} / 2 \pi M \mathbb{Z}\right)} \lesssim M\|\omega\|_{H^{k}\left(\mathbb{R}^{2} \times \mathbb{R} / 2 \pi M \mathbb{Z}\right)}
$$

and

$$
\left\|\varphi \nabla \Delta_{w}^{-1} \tilde{A}_{0}^{*} \omega\right\|_{H^{k+2}\left(\mathbb{R}^{2} \times \mathbb{R} / 2 \pi M \mathbb{Z}\right)} \lesssim M\|\omega\|_{H^{k}\left(\mathbb{R}^{2} \times \mathbb{R} / 2 \pi M \mathbb{Z}\right)}
$$

for scalar $\omega \in H^{k}\left(\mathbb{R}^{2} \times \mathbb{R} / 2 \pi M \mathbb{Z}\right)$ supported in the support of $\varphi$.

If $\omega$ is bounded in $H^{k}\left(\mathbb{R}^{2} \times \mathbb{R} / 2 \pi M \mathbb{Z}\right.$ ), then by (8.10) (replacing $\mathbb{R} / \mathbb{Z}$ with $\mathbb{R} / 2 \pi M \mathbb{Z})$ we see that $\tilde{A}_{0} \Delta_{w}^{-1} \nabla \omega$ is bounded in $\dot{H}^{k+2}\left(\mathbb{R}^{2} \times \mathbb{R} / 2 \pi M \mathbb{Z}\right)$, but from 
(8.9) we also see that this function is supported in $B_{\mathbb{R}^{2} \times \mathbb{R} / 2 \pi M \mathbb{Z}}(0,100)$. From this and the fundamental theorem of calculus we see that $\tilde{A}_{0} \Delta_{w}^{-1} \nabla \omega$ is in fact bounded in $H^{k+2}\left(\mathbb{R}^{2} \times \mathbb{R} / 2 \pi M \mathbb{Z}\right)$, giving (9.7). A similar argument gives (9.8), completing the proof of (1.20).

Now we show (1.19). From the explicit formula $\frac{1}{4 \pi|x-y|}$ for the Newton potential kernel of $\Delta^{-1}$, we see that the contribution of the $\tilde{\eta}^{-1} \Delta^{-1}$ term in (9.4) is acceptable. The remaining terms in (9.4) only give a contribution to the kernel when $x, y=O_{M}(1)$. The contribution of $\varphi \tilde{\eta}^{-1} \Delta^{-1} \varphi$ can then be seen to also be acceptable by the Leibniz rule. By further application of the Leibniz rule and the chain rule, it thus suffices to show that the kernel $K\left(w, w^{\prime}\right)$ of $A^{\prime}$ obeys the estimates

$$
\left|\nabla_{w}^{i} \nabla_{w^{\prime}}^{j} K\left(w, w^{\prime}\right)\right| \lesssim_{M}\left|w-w^{\prime}\right|^{-i-j-1}
$$

whenever $0 \leqslant i, j \leqslant M$ with $i+j \geqslant 1$. But this follows from the arguments used to prove Proposition 8.2.

Proposition 9.2 For any $\omega \in C_{c}^{\infty} \cap B_{2}(\mathcal{M})$, we have

$$
\int_{\mathcal{M}}\langle\omega, \tilde{A} \omega\rangle d \text { vol }=\left(1+O\left(\frac{1}{\log M}\right)\right)\|\omega\|_{\dot{H}^{-1}(\mathcal{M})}^{2} .
$$

Proof From Fourier analysis we may write $\omega=d v$ for some $v \in L^{2} \cap \Lambda_{1}(\mathcal{M})$ with

$$
\|v\|_{L^{2}(\mathcal{M})}=\|\omega\|_{\dot{H}^{-1}(\mathcal{M})}
$$

From integration by parts, we have

$$
\int_{\mathcal{M}}\left\langle d v, \tilde{\eta}^{-1} \Delta^{-1} d v\right\rangle d \mathrm{vol}=\|v\|_{L^{2}(\mathcal{M})}^{2}
$$

so by (9.4) and the triangle inequality it suffices to show that

$$
\int_{\mathcal{M}}\left\langle d v,\left(I^{*} A^{\prime} I-\varphi \tilde{\eta}^{-1} \Delta^{-1} \varphi\right) d v\right\rangle d \text { vol }=O\left(\frac{1}{\log M}\|v\|_{L^{2}(\mathcal{M})}^{2}\right) .
$$

From the Newton formula

$$
\Delta^{-1} f(x)=\int_{\mathcal{M}} \frac{f(y)}{4 \pi|x-y|} d \operatorname{vol}(y)
$$

one has

$$
\begin{gathered}
\int_{\mathcal{M}}\left\langle d v, \varphi \tilde{\eta}^{-1} \Delta^{-1} \varphi d v\right\rangle d \operatorname{vol}=\int_{\mathcal{M}} \int_{\mathcal{M}} \frac{\left\langle\varphi d v(x), \tilde{\eta}^{-1} \varphi d v(y)\right\rangle}{4 \pi|x-y|} d \operatorname{vol}(x) d \operatorname{vol}(y) \\
=\int_{\mathbb{R}^{2} \times \mathbb{R} / 2 \pi M \mathbb{Z}} \int_{\mathbb{R}^{2} \times \mathbb{R} / 2 \pi M \mathbb{Z}} \frac{\left\langle I d v(w),\left(\tilde{\eta}^{\prime}\right)^{-1} I d v\left(w^{\prime}\right)\right\rangle}{4 \pi\left|w_{\mathrm{rsc}}-w_{\mathrm{rsc}}^{\prime}\right|} d \operatorname{vol}(w) d \operatorname{vol}\left(w^{\prime}\right)
\end{gathered}
$$


where $\eta^{\prime}$ is the metric on the support of $I \omega$ formed by pulling back the Euclidean metric $\eta$, thus by (9.3)

$$
\left(d \eta^{\prime}\right)^{2}=\left(d w^{1}\right)^{2}+\left(1+\frac{2 w^{2}}{M}\right)^{-1}\left(d w^{2}\right)^{2}+\left(1+\frac{2 w^{2}}{M}\right)\left(d w^{3}\right)^{2}
$$

Meanwhile, from (9.5) we have

$$
\begin{aligned}
& \int_{\mathcal{M}}\left\langle d v, I^{*} A^{\prime} I d v\right\rangle d \text { vol }=\int_{\mathbb{R}^{2} \times \mathbb{R} / 2 \pi M \mathbb{Z}}\left\langle I d v, A^{\prime} I d v\right\rangle d \text { vol } \\
& =-2 \int_{\mathbb{R}^{2} \times \mathbb{R} / 2 \pi M \mathbb{Z}}\left\langle I d v, \tilde{A}_{0} \Delta_{w}^{-1}\left(\partial_{1}(I d v)_{13}+\partial_{2}(I d v)_{23}\right) \frac{d}{d w^{1}} \wedge \frac{d}{d w^{2}}\right\rangle d \text { vol } \\
& \quad+\int_{\mathbb{R}^{2} \times \mathbb{R} / 2 \pi M \mathbb{Z}} \int_{\mathbb{R}^{2} \times \mathbb{R} / 2 \pi M \mathbb{Z}} \\
& K_{2 \pi M}\left(w-w^{\prime}\right)\left\langle I d v(w), \tilde{\eta}^{-1} I d v\left(w^{\prime}\right)\right\rangle d \operatorname{vol}(w) d \operatorname{vol}\left(w^{\prime}\right),
\end{aligned}
$$

where by abuse of notation $\eta$ now also denotes the Euclidean metric on $\mathbb{R}^{2} \times \mathbb{R} / 2 \pi M \mathbb{Z}$, and $K_{2 \pi} M$ was defined in (9.6). Thus by the triangle inequality it will suffice to establish the estimates

$$
\begin{aligned}
& \int_{\mathbb{R}^{2} \times \mathbb{R} / 2 \pi M \mathbb{Z}}\left\langle I d v, \tilde{A}_{0} \Delta_{w}^{-1}\left(\partial_{1}(I d v)_{13}+\partial_{2}(I d v)_{23}\right) \frac{d}{d w^{1}} \wedge \frac{d}{d w^{2}}\right\rangle d \text { vol } \\
& =O\left(\frac{1}{\log M}\|v\|_{L^{2}(\mathcal{M})}^{2}\right)
\end{aligned}
$$

and

$$
\begin{aligned}
& \int_{\mathbb{R}^{2} \times \mathbb{R} / 2 \pi M \mathbb{Z}} \int_{\mathbb{R}^{2} \times \mathbb{R} / 2 \pi M \mathbb{Z}} \\
& \frac{\left\langle I d v(w),\left(\tilde{\eta}^{\prime}\right)^{-1} I d v\left(w^{\prime}\right)\right\rangle}{4 \pi\left|w_{\mathrm{rsc}}-w_{\mathrm{rsc}}^{\prime}\right|}-K_{2 \pi M}\left(w-w^{\prime}\right)\left\langle\operatorname{Id} v(w), \tilde{\eta}^{-1} I d v\left(w^{\prime}\right)\right\rangle \\
& d \operatorname{vol}(w) d \operatorname{vol}\left(w^{\prime}\right)=O\left(\frac{1}{\log M}\|v\|_{L^{2}(\mathcal{M})}^{2}\right) .
\end{aligned}
$$

The bound (9.12) follows easily from (8.11) (with the factor of $\frac{1}{\log M}$ improved to $\frac{1}{M}$ ), so we turn to (9.13). Forming the tensor kernel

$$
L\left(w, w^{\prime}\right):=\frac{1}{4 \pi\left|w_{\mathrm{rsc}}-w_{\mathrm{rsc}}^{\prime}\right|} \tilde{\eta}^{\prime}\left(w^{\prime}\right)^{-1}-K_{2 \pi M}\left(w-w^{\prime}\right) \tilde{\eta}\left(w^{\prime}\right)^{-1}
$$

we see from integration by parts, the chain rule and duality that it suffices to prove the operator norm bound 


$$
\begin{aligned}
& \left\|\delta \int_{\mathbb{R}^{2} \times \mathbb{R} / 2 \pi M \mathbb{Z}} \varphi(\cdot) L\left(\cdot, w^{\prime}\right) \varphi\left(w^{\prime}\right) d v\left(w^{\prime}\right) d \operatorname{vol}\left(w^{\prime}\right)\right\|_{L^{2}\left(\mathbb{R}^{2} \times \mathbb{R} / 2 \pi M \mathbb{Z}\right)} \\
& \quad \lesssim \frac{1}{\log M}\|v\|_{L^{2}\left(\mathbb{R}^{2} \times \mathbb{R} / 2 \pi M \mathbb{Z}\right)}
\end{aligned}
$$

for all $v \in L^{2} \cap \Lambda_{1}\left(\mathbb{R}^{2} \times \mathbb{R} / 2 \pi M \mathbb{Z}\right)$.

To prove this estimate, we first claim the kernel estimates

$$
\left|\nabla_{w}^{i} \nabla_{w^{\prime}}^{j} L\left(w, w^{\prime}\right)\right| \lesssim i, j \frac{1}{\sqrt{M}} \frac{1}{\left|w-w^{\prime}\right|^{i+j}}\left(\frac{1}{\sqrt{M}}+\frac{1}{\left|w-w^{\prime}\right|}\right)
$$

for distinct $w, w^{\prime} \in Q$ and $i, j \geqslant 0$.

From (9.11) we have

$$
\left|\tilde{\eta}^{\prime}\left(w^{\prime}\right)^{-1}-\tilde{\eta}\left(w^{\prime}\right)\right| \lesssim \frac{1}{\sqrt{M}}
$$

for $w^{\prime} \in Q$, and more generally

$$
\left|\nabla_{w^{\prime}}^{j}\left(\tilde{\eta}^{\prime}\left(w^{\prime}\right)^{-1}-\tilde{\eta}\left(w^{\prime}\right)\right)\right| \lesssim j \frac{1}{\sqrt{M}} \frac{1}{M^{j}}
$$

for $w^{\prime} \in Q$ and $j \geqslant 0$. Also, from many applications of the chain rule one has

$$
\left|\nabla_{w}^{i} \nabla_{w^{\prime}}^{j} \frac{1}{4 \pi\left|w_{\mathrm{rsc}}-w_{\mathrm{rsc}}^{\prime}\right|}\right| \lesssim_{i, j} \frac{1}{|x-y|^{1+i+j}}
$$

for $w, w^{\prime} \in Q$ and $i, j \geqslant 0$, and hence by the product rule

$$
\mid \nabla_{w}^{i} \nabla_{w^{\prime}}^{j}\left(\frac{1}{4 \pi\left|w_{\mathrm{rsc}}-w_{\mathrm{rsc}}^{\prime}\right|}\left(\tilde{\eta}^{\prime}\left(w^{\prime}\right)^{-1}-\tilde{\eta}\left(w^{\prime}\right)^{-1}\right) \mid \lesssim_{i, j} \frac{1}{\sqrt{M}} \frac{1}{\left|w-w^{\prime}\right|^{1+i+j}} .\right.
$$

Thus by the triangle inequality it suffices to show that

$$
\begin{aligned}
& \left|\nabla_{w}^{i} \nabla_{w^{\prime}}^{j}\left(\frac{1}{4 \pi\left|w_{\mathrm{rsc}}-w_{\mathrm{rsc}}^{\prime}\right|} \tilde{\eta}\left(w^{\prime}\right)^{-1}-K_{2 \pi M}\left(w-w^{\prime}\right) \tilde{\eta}\left(w^{\prime}\right)^{-1}\right)\right| \\
& \quad \lesssim_{i, j} \frac{1}{\sqrt{M}} \frac{1}{\left|w-w^{\prime}\right|^{i+j}}\left(\frac{1}{\sqrt{M}}+\frac{1}{\left|w-w^{\prime}\right|}\right) .
\end{aligned}
$$

As $\tilde{\eta}$ is constant coefficient, we can drop the $\tilde{\eta}\left(w^{\prime}\right)^{-1}$ factor, thus we reduce to establishing

$$
\left|\nabla_{w}^{i} \nabla_{w^{\prime}}^{j}\left(\frac{1}{4 \pi\left|w_{\mathrm{rsc}}-w_{\mathrm{rsc}}^{\prime}\right|}-K_{2 \pi M}\left(w-w^{\prime}\right)\right)\right| \lesssim_{i, j} \frac{1}{\sqrt{M}} \frac{1}{\left|w-w^{\prime}\right|^{i+j}}\left(\frac{1}{\sqrt{M}}+\frac{1}{\left|w-w^{\prime}\right|}\right)
$$

for $w, w^{\prime} \in Q$ and $i, j \geqslant 0$. 
We first dispose of the case where $w, w^{\prime}$ are very far apart in the sense that $\left|w-w^{\prime}\right| \geqslant$ $M / 10$. From (9.6) (and recalling that the $w^{1}$ and $w^{2}$ components of $w, w^{\prime}$ are $O(\sqrt{M})$ ) we see that

$$
\left|K_{2 \pi M}\left(w-w^{\prime}\right)\right| \lesssim \frac{1}{M}
$$

which by the harmonicity of $K_{2 \pi} M$ implies that

$$
\left|\nabla_{w}^{i} \nabla_{w^{\prime}}^{j} K_{2 \pi M}\left(w-w^{\prime}\right)\right| \lesssim_{i, j} \frac{1}{M^{1+i+j}}
$$

Similarly, as the map $w \mapsto w_{\text {rsc }}$ is bilipschitz with all derivatives bounded, we have

$$
\left|\frac{1}{4 \pi\left|w_{\mathrm{rsc}}-w_{\mathrm{rsc}}^{\prime}\right|}\right| \lesssim \frac{1}{M}
$$

and more generally

$$
\left|\nabla_{w}^{i} \nabla_{w^{\prime}}^{j} \frac{1}{4 \pi\left|w_{\mathrm{rsc}}-w_{\mathrm{rsc}}^{\prime}\right|}\right| \lesssim_{i, j} \frac{1}{M^{1+i+j}},
$$

and so (9.16) follows from the triangle inequality in this case.

Henceforth we suppose that $\left|w-w^{\prime}\right|<M / 10$. From (9.6) we now have

$$
\left|K_{2 \pi M}\left(w-w^{\prime}\right)-\frac{1}{4 \pi\left|w-w^{\prime}\right|}\right| \lesssim \frac{1}{M}
$$

which by harmonicity implies

$$
\left|\nabla_{w}^{i} \nabla_{w^{\prime}}^{j}\left(K_{2 \pi M}\left(w-w^{\prime}\right)-\frac{1}{4 \pi\left|w-w^{\prime}\right|}\right)\right| \lesssim i, j \frac{1}{M} \frac{1}{\left|w-w^{\prime}\right|^{i+j}} .
$$

Thus by the triangle inequality, it suffices to show that

$$
\left|\nabla_{w}^{i} \nabla_{w^{\prime}}^{j}\left(\frac{1}{\left|w_{\mathrm{rsc}}-w_{\mathrm{rsc}}^{\prime}\right|}-\frac{1}{\left|w-w^{\prime}\right|}\right)\right| \lesssim i, j \frac{1}{\sqrt{M}} \frac{1}{\left|w-w^{\prime}\right|^{i+j}}\left(\frac{1}{\sqrt{M}}+\frac{1}{\left|w-w^{\prime}\right|}\right) .
$$

We divide into two cases, depending on whether $\left|w-w^{\prime}\right|$ is less than $\sqrt{M}$ or not. First suppose that $\left|w-w^{\prime}\right| \leqslant \sqrt{M}$, thus $w, w^{\prime}$ both lie in $B_{\mathbb{R}^{2} \times \mathbb{R} / 2 \pi M \mathbb{Z}}\left(w_{0}, \sqrt{M}\right)$ for some $w_{0} \in Q$. Let $B$ denote the convex region

$$
B:=\left\{u \in B_{\mathbb{R}^{3}}(0,1): w_{0}+\sqrt{M} u \in Q\right\},
$$

and let $f: B \rightarrow \mathbb{R}^{3}$ be the map

$$
f(u):=\frac{\left(w_{0}+\sqrt{M} u\right)_{\mathrm{rsc}}-\left(w_{0}\right)_{\mathrm{rsc}}}{\sqrt{M}}
$$


then it is easy to see that $f$ is bilipschitz on $B$ with constants comparable to 1 , and from Taylor expansion we see that

$$
\nabla_{u}^{i}(f(u)-u)=O_{i}\left(\frac{1}{\sqrt{M}}\right)
$$

on $B$ for all $i \geqslant 0$. In particular, we have for distinct $u, v \in B$ that $|f(u)-f(v)|$ is comparable to $|u-v|$, and from several applications of the chain rule (and writing $f(u)=u+\frac{1}{\sqrt{M}} g(u)$ for some function $g$ with all derivatives bounded on $B$ ) we have

$$
\nabla_{u}^{i} \nabla_{v}^{j}\left(\frac{1}{|f(u)-f(v)|}-\frac{1}{|u-v|}\right)=O_{i, j}\left(\frac{1}{\sqrt{M}|u-v|^{1+i+j}}\right)
$$

for $i, j \geqslant 0$. Setting $w=w_{0}+\sqrt{M} u$ and $w^{\prime}=w_{0}+\sqrt{M} v$, we obtain (9.17) when $\left|w-w^{\prime}\right| \leqslant \sqrt{M}$.

To complete the proof of (9.15), we need to establish (9.17) in the case $\sqrt{M}<$ $\left|w-w^{\prime}\right| \leqslant M / 10$. Set $R:=\left|w-w^{\prime}\right| / \sqrt{M}$, then $1 \leqslant R \leqslant \sqrt{M} / 10$, and $w, w^{\prime}$ both lie in $B_{\mathbb{R}^{2} \times \mathbb{R} / 2 \pi M \mathbb{Z}}\left(w_{0}, R \sqrt{M}\right)$ for some $w_{0} \in Q$. Setting $B_{R}$ to be the convex region

$$
B_{R}:=\left\{u \in B_{\mathbb{R}^{3}}(0, R): w_{0}+\sqrt{M} u \in Q\right\}
$$

and defining $f$ by (9.18) as before, one has from Taylor expansion that

$$
f(u)=u+O\left(\frac{R^{2}}{\sqrt{M}}\right)
$$

and more generally

$$
\nabla_{u}^{i}(f(u)-u)=O_{i}\left(\frac{R^{2-i}}{\sqrt{M}}\right)
$$

on $B_{R}$ for all $i \geqslant 0$. As before, $|f(u)-f(v)|$ is comparable to $|u-v|$. By many applications of the chain rule, we have

$$
\nabla_{u}^{i} \nabla_{v}^{j}\left(\frac{1}{|f(u)-f(v)|}-\frac{1}{|u-v|}\right)=O_{i, j}\left(\frac{R}{\sqrt{M}}|u-v|^{-1-i-j}\right)
$$

for $i, j \geqslant 0$. Setting $w=w_{0}+\sqrt{M} u$ and $w^{\prime}=w_{0}+\sqrt{M} v$, so that $|u-v|$ is comparable to $R$, we obtain (9.17) when $\sqrt{M}<\left|w-w^{\prime}\right| \leqslant M / 10$.

This completes the proof of (9.15) in all cases. We now return to the proof of (9.14). Let $\pi: \mathbb{R}^{2} \times \mathbb{R} / 2 \pi M \mathbb{Z} \rightarrow \mathbb{R}^{2}$ denote the projection map $\pi:\left(w^{1}, w^{2}, w^{3}\right) \mapsto$ $\left(w^{1}, w^{2}\right)$. We smoothly partition $L=L_{1}+L_{2}$, where $L_{1}\left(w, w^{\prime}\right)$ is the "local" part of $L\left(w, w^{\prime}\right)$ smoothly restricted to the region where $\left|w-w^{\prime}\right| \leqslant \log M \min (|\pi(w)|$, 
$\left.\left|\pi\left(w^{\prime}\right)\right|\right)$, and $L_{1}$ is the "global" part, restricted to the region where $\left|w-w^{\prime}\right| \gg$ $\log M \min \left(|\pi(w)|,\left|\pi\left(w^{\prime}\right)\right|\right)$. More explicitly, we can set

$$
L_{1}\left(w, w^{\prime}\right):=L\left(w, w^{\prime}\right) \chi\left(\frac{w-w^{\prime}}{|\pi(w)| \log M}\right) \times\left(\frac{w-w^{\prime}}{\left|\pi\left(w^{\prime}\right)\right| \log M}\right)
$$

where $\chi: \mathbb{R}^{2} \rightarrow[0,1]$ is a smooth function supported on $B_{\mathbb{R}^{2}}(0,1)$ that equals one on $B_{\mathbb{R}^{2}}(0,1 / 2)$, and set $L_{2}:=L-L_{1}$. By the triangle inequality, it thus suffices to establish the bounds

$$
\begin{aligned}
& \left\|\delta \int_{\mathbb{R}^{2} \times \mathbb{R} / 2 \pi M \mathbb{Z}} \varphi(\cdot) L_{l}\left(\cdot, w^{\prime}\right) \varphi\left(w^{\prime}\right) d v\left(w^{\prime}\right) d \operatorname{vol}\left(w^{\prime}\right)\right\|_{L^{2}\left(\mathbb{R}^{2} \times \mathbb{R} / 2 \pi M \mathbb{Z}\right)} \\
& \quad \lesssim \frac{1}{\log M}\|v\|_{L^{2}\left(\mathbb{R}^{2} \times \mathbb{R} / 2 \pi M \mathbb{Z}\right)}
\end{aligned}
$$

for $l=1,2$.

In the $l=1$ case, we note that as $L_{l}$ is supported in the regime where $\left|w-w^{\prime}\right| \lesssim$ $|\pi(w)| \log M,\left|\pi\left(w^{\prime}\right)\right| \log M \lesssim \sqrt{M} \log M$, and we see from (9.15) and the product rule that we have the Calderón-Zygmund bounds

$$
\left|\nabla_{w} \nabla_{w^{\prime}}\left(\varphi(w) L_{l}\left(w, w^{\prime}\right) \varphi\left(w^{\prime}\right)\right)\right| \lesssim \frac{\log O(1) M}{\sqrt{M}} \frac{1}{\left|w-w^{\prime}\right|^{3}}
$$

and

$$
\left|\nabla_{w, w^{\prime}} \nabla_{w} \nabla_{w^{\prime}}\left(\varphi(w) L_{l}\left(w, w^{\prime}\right) \varphi\left(w^{\prime}\right)\right)\right| \lesssim \frac{\log O(1) M}{\sqrt{M}} \frac{1}{\left|w-w^{\prime}\right|^{4}}
$$

for $w \neq w^{\prime}$. Also, the operator that maps $v$ to

$$
\delta \int_{\mathbb{R}^{2} \times \mathbb{R} / 2 \pi M \mathbb{Z}} \varphi(\cdot) L_{l}\left(\cdot, w^{\prime}\right) \varphi\left(w^{\prime}\right) d v\left(w^{\prime}\right) d \operatorname{vol}\left(w^{\prime}\right)
$$

clearly annihilates the constant function 1, as does its adjoint. Applying the $T$ (1) theorem of David and Journé [31], we obtain the $l=1$ case of (9.19) (with the $\frac{1}{\log M}$ factor improved to $\frac{\log O(1) M}{\sqrt{M}}$ ).

Now we handle the $l=2$ case. From (9.15) and the product rule, we have the bounds

$$
\begin{aligned}
\left|\nabla_{w} \nabla_{w^{\prime}}\left(\varphi(w) L_{l}\left(w, w^{\prime}\right) \varphi\left(w^{\prime}\right)\right)\right| \lesssim & \frac{1}{\sqrt{M}}\left(\frac{1}{\sqrt{M}}+\frac{1}{\left|w-w^{\prime}\right|}\right) \\
& \times \frac{1}{\log ^{2} M} \frac{1}{1+|\pi(w)|} \frac{1}{1+\left|\pi\left(w^{\prime}\right)\right|}
\end{aligned}
$$


since any factor of $\frac{1}{\left|w-w^{\prime}\right|}$ that comes when a derivative falls on $L_{l}$ can be replaced instead by $\frac{1}{\log M} \frac{1}{1+|\pi(w)|}$ or $\frac{1}{\log M} \frac{1}{1+\left|\pi\left(w^{\prime}\right)\right|}$. In particular, we have the estimates

$$
\int_{\mathbb{R}^{2} \times \mathbb{R} / 2 \pi M \mathbb{Z}}\left|\nabla_{w} \nabla_{w^{\prime}}\left(\varphi(w) L_{l}\left(w, w^{\prime}\right) \varphi\left(w^{\prime}\right)\right)\right| \frac{d \operatorname{vol}\left(w^{\prime}\right)}{1+\left|\pi\left(w^{\prime}\right)\right|} \lesssim \frac{1}{\log M} \frac{1}{1+|\pi(w)|}
$$

for all $w$, and

$$
\int_{\mathbb{R}^{2} \times \mathbb{R} / 2 \pi M \mathbb{Z}}\left|\nabla_{w} \nabla_{w^{\prime}}\left(\varphi(w) L_{l}\left(w, w^{\prime}\right) \varphi\left(w^{\prime}\right)\right)\right| \frac{d \operatorname{vol}(w)}{1+|\pi(w)|} \lesssim \frac{1}{\log M} \frac{1}{1+\left|\pi\left(w^{\prime}\right)\right|}
$$

for all $w^{\prime}$. The $l=2$ case of (9.19) then follows from the weighted Schur test (after integrating by parts to move all derivatives onto $\left.\varphi(w) L_{l}\left(w, w^{\prime}\right) \varphi\left(w^{\prime}\right)\right)$.

Now we prove Theorem 1.13. As in the previous section, let $\theta_{0}: \mathbb{R}^{2} \rightarrow \mathbb{R}$ be initial data of the type in Proposition 6.1; we can choose $\theta_{0}$ so that it is supported in the ball $B_{\mathbb{R}^{2}}(0,200)$. Let $\tilde{\theta}_{0}: \mathcal{M} \rightarrow \mathbb{R}$ be the lift of $\theta_{0}$ to $\mathcal{M}$ defined by setting

$$
\tilde{\theta}_{0}\left(\left(w^{1}, w^{2}, w^{3}\right)_{\mathrm{rsc}}\right):=\theta_{0}\left(w^{1}, w^{2}\right)
$$

in the region (9.2), with $\tilde{\theta}_{0}$ vanishing outside of (9.2). Clearly $\tilde{\theta}$ is smooth and supported in the set $\left\{\left(w^{1}, w^{2}, w^{3}\right)_{\text {rsc }}:\left|w^{1}\right|,\left|w^{2}\right| \leqslant 200\right\}$. We define the initial data $\omega_{0} \in$ $C_{c}^{\infty} \cap B_{2}(\mathcal{M})$ by the formula

$$
\omega_{0}:=d \tilde{\theta}_{0} \wedge d w^{3}=d\left(\tilde{\theta}_{0} d w^{3}\right)
$$

noting that the 1 -form $d w^{3}$ is well-defined on the support of $\tilde{\theta}_{0}$. This is clearly a closed 2-form. We now claim (for $M$ sufficiently large) that Theorem 1.13 holds with this choice of initial data $\omega_{0}$ and with the operator $\tilde{A}$ constructed above as vector potential operator. We have already verified that $\tilde{A}$ is 100 -reasonable, formally selfadjoint, and obeys (1.22) (if $M$ is sufficiently large depending on $\varepsilon$ ). Thus, the only way that Theorem 1.13 can still fail is if there is a solution $\omega \in X^{10,2}, u \in Y^{10,2}$ to the generalised Euler equations with vector potential operator $\tilde{A}$ and initial vorticity $\omega_{0}$ on the time interval $[0,1]$.

Suppose for contradiction that this is the case. Observe that $\omega_{0}$ is invariant with respect to rotations around the $x^{3}$ axis (9.1) (which, in the region (9.2), corresponds to translations in the $w^{3}$ direction), and that $\tilde{A}$ commutes with these rotations. Thus, if $\omega, u$ solve the generalised Euler equations with initial data $\omega_{0}$, then so do any rotations of $\omega, u$ around the $x^{3}$ axis. Applying the uniqueness component of Theorem 1.9 , we conclude that $\omega, u$ are invariant with respect to rotations around the $x^{3}$ axis. In particular, in the region (9.2), we have

$$
\partial_{3} \omega=0 ; \quad \partial_{3} u=0
$$

in the $\left(w^{1}, w^{2}, w^{3}\right)_{\text {rsc }}$ coordinate system. 
We define the scalar field $\tilde{\theta}:[0,1] \times \mathcal{M} \rightarrow \mathbb{R}$ by solving the transport equation (8.17) with initial data $\tilde{\theta}=\tilde{\theta}_{0}$. Again, there is no difficulty defining $\tilde{\theta}$, and it is continuously differentiable in both space and time, and compactly supported in space. Since $\tilde{\theta}$ and $u$ are invariant with respect to rotations around the $x^{3}$ axis, $\tilde{\theta}$ is also.

Next, we claim that $\omega$ stays well within the region (9.2) and obeys the analogue of Proposition 8.4:

Proposition 9.3 For each $0 \leqslant t \leqslant 1$, let $\Omega(t)$ be the subset of (9.2) defined by

$$
\Omega(t):=\left\{\left(w^{1}, w^{2}, w^{3}\right)_{\mathrm{rsc}}:\left|w^{1}\right|,\left|w^{2}\right| \leqslant 300+t\right\} .
$$

Then $\omega(t)$ and $\tilde{\theta}(t)$ are supported in $\Omega(t)$ for all $0 \leqslant t \leqslant 1$, and we have

$$
\begin{aligned}
\omega & =d \tilde{\theta} \wedge d w^{3} \\
& =\partial_{1} \tilde{\theta} d w^{1} \wedge d w^{3}+\partial_{2} \tilde{\theta} d w^{2} \wedge d w^{3} .
\end{aligned}
$$

Proof We again use the barrier method. Since $u$ lies in $Y^{10,2}$, it is bounded, and hence $\omega$ is transported at bounded speed. Suppose the first claim fails, thus $\omega(t)$ or $\tilde{\theta}(t)$ is not supported in $\Omega(t)$ for some $0 \leqslant t \leqslant 1$. Let $0 \leqslant T \leqslant 1$ be the infimum of all the times $t$ in which $\omega(t)$ or $\tilde{\theta}(t)$ is not supported in $\Omega(t)$. Since this is a closed condition, we have $T<1$. Since $\omega(0)$ and $\tilde{\theta}(0)$ are supported in the interior of $\Omega(0)$ and is transported at bounded speed, we have $T>0$.

For times $t \in[0, T]$, set $\alpha$ to be the 2-form

$$
\alpha:=\omega-d \tilde{\theta} \wedge d w^{3}
$$

then $\alpha$ is continuously differentiable in space and time and supported in (9.2). From (8.16) we know that $\alpha(0)=0$. As in the proof of Proposition 8.4, we use (1.10), (8.17) to compute

$$
\begin{aligned}
\left(\partial_{t}+\mathcal{L}_{u}\right) \alpha & =\left(\partial_{t}+\mathcal{L}_{u}\right) \alpha-\left(\partial_{t}+\mathcal{L}_{u}\right)\left(d \tilde{\theta} \wedge d w^{3}\right) \\
& =0-d 0 \wedge d w^{3}-d \tilde{\theta} \wedge d \mathcal{L}_{u} w^{3} \\
& =-d \tilde{\theta} \wedge d u^{3}
\end{aligned}
$$

where $u^{3}$ is the $\frac{d}{d w^{3}}$ component of $u$. On the other hand, from (1.11) and (9.4), (9.5) (noting that $\tilde{\eta}$ equals 1 on the support of $\omega$ or $\tilde{\theta}$ for times in $[0, T]$ ) we have

$$
\begin{aligned}
u^{3}= & \partial_{1} \partial_{1} \Delta_{w}^{-1} \tilde{A}_{0}^{*} \omega_{12}+\partial_{2} \partial_{2} \Delta_{w}^{-1} \tilde{A}_{0}^{*} \omega_{12} \\
& +\partial_{1} \Delta_{w}^{-1} \omega_{13}+\partial_{2} \Delta_{w}^{-1} \omega_{23} \\
= & \tilde{A}_{0}^{*} \alpha_{12}+\partial_{1} \Delta^{-1} \partial_{1} \tilde{\theta}+\partial_{2} \Delta_{w}^{-1} \partial_{2} \tilde{\theta} \\
& +\partial_{1} \Delta^{-1} \alpha_{13}+\partial_{2} \Delta_{w}^{-1} \alpha_{23} \\
= & \left(\tilde{A}_{0}^{*} \alpha_{12}+\partial_{1} \Delta_{w}^{-1} \alpha_{13}+\partial_{2} \Delta_{w}^{-1} \alpha_{23}\right)-\tilde{\theta}
\end{aligned}
$$


where all derivatives and components are with respect to the $\left(w^{1}, w^{2}, w^{3}\right)_{\text {rsc }}$ coordinate system. Repeating the arguments in Theorem 8.4 verbatim, we thus have $\alpha(t)=0$ for all $0 \leqslant t \leqslant T$. Thus we have

$$
\omega=d \tilde{\theta} \wedge d w^{3}
$$

for times $0 \leqslant t \leqslant T$. In particular, $\omega_{12}$ vanishes.

By continuity and a compactness argument, there must exist a point $\left(w^{1}, w^{2}, w^{3}\right)_{\text {rsc }}$ on the boundary of $\Omega(T)$ which is also on the boundary of the support of $\omega(T)$ or $\tilde{\theta}(T)$, but such that the support of $\omega(t)$ or $\tilde{\theta}(t)$ escapes $\Omega(t)$ in any given neighbourhood of $\left(w^{1}, w^{2}, w^{3}\right)_{\text {rsc }}$ for times $t>T$ arbitrarily close to $T$.

Now we compute the vector potential $\tilde{A} \omega$ and velocity field $u$ at time $T$ and in a sufficiently small neighbourhood of this point $\left(w^{1}, w^{2}, w^{3}\right)_{\text {rsc }}$. In this neighbourhood and on the support of $\omega(T)$, the cutoff $\tilde{\eta}$ equals 1 , and from (8.9) we see that $A_{0}$ and $A_{0}^{*}$ vanish in this neighbourhood. From (9.4), (9.5) and the vanishing of $\omega_{12}$ we thus have

$$
\tilde{A} \omega=\Delta_{w}^{-1} \omega_{13} \frac{d}{d w^{1}} \wedge \frac{d}{d w^{3}}+\Delta_{w}^{-1} \omega_{23} \frac{d}{d w^{2}} \wedge \frac{d}{d w^{3}}
$$

in this neighbourhood, after abusing notation and identifying this neighbourhood with a subset of $\mathbb{R}^{2} \times \mathbb{R} / 2 \pi M \mathbb{Z}$. Since $\omega_{13}, \omega_{23}$ are invariant with respect to translations in the $w^{3}$ neighbourhood, we conclude that $\delta \tilde{A} \omega$ has vanishing $\frac{d}{d w^{1}}$ and $\frac{d}{d w^{2}}$ components in this neighbourhood, thus the velocity field $u$ is parallel to $\frac{d}{d w^{3}}$. But since $\Omega(T)$ is invariant in the $\frac{d}{d w^{3}}$ direction and is expanding outwards in the other two directions, we see from the transport equations for $\omega(t)$ and $\tilde{\theta}(t)$ that for $t>T$ sufficiently close to $T, \omega(t)$ and $\tilde{\theta}(t)$ are supported inside $\Omega(t)$ in this neighbourhood, contradicting the construction of $\left(w^{1}, w^{2}, w^{3}\right)_{\text {rsc }}$. Thus $\omega(t)$ and $\tilde{\theta}(t)$ are supported in $\Omega(t)$ for all $0 \leqslant t \leqslant 1$. Repeating the above arguments we then obtain the second claim of the proposition.

If we insert the above proposition back into (9.4), (9.5), noting again that $\tilde{\eta}$ equals 1 on the support of $\omega$ or $\tilde{\theta}$, we have

$$
\begin{aligned}
\tilde{A} \omega: & =-\tilde{A}_{0} \Delta_{w}^{-1}\left(\partial_{1} \partial_{1} \tilde{\theta}+\partial_{2} \partial_{2} \tilde{\theta}\right) \frac{d}{d w^{1}} \wedge \frac{d}{d w^{2}} \\
& +\Delta_{w}^{-1} \partial_{1} \tilde{\theta} \frac{d}{d w^{1}} \wedge \frac{d}{d w^{3}} \\
& +\Delta_{w}^{-1} \partial_{2} \tilde{\theta} \frac{d}{d w^{2}} \wedge \frac{d}{d w^{3}} .
\end{aligned}
$$

Repeating the arguments of the previous section verbatim, we see that (in $\left(w^{1}, w^{2}, w^{3}\right)_{\text {rsc }}$ coordinates) $\tilde{\theta}$ is the lift of a continuously differentiable, compactly supported function $\theta:[0,1] \times \mathbb{R}^{2} \rightarrow \mathbb{R}$ that contradicts Proposition 6.1, as required. 
Acknowledgements The author is supported by NSF Grant DMS-1266164 and by a Simons Investigator Award. The author thanks Nets Katz for many useful conversations and encouragement, and Peter Constantin and the anonymous referees for many helpful suggestions and comments.

\section{References}

1. Arnold, V.: Sur la géométrie différentielle des groupes de Lie de dimension infinie et ses applications à l'hydrodynamique des fluides parfaits. Ann. Inst. Fourier (Grenoble) 16, 319-361 (1966)

2. Aubin, T.: A Course in Differential Geometry. Graduate Studies in Mathematics, vol. 27. American Mathematical Society, Providence (2001)

3. Beale, J.T., Kato, T., Majda, A.: Remarks on the breakdown of smooth solutions for the 3-D Euler equations. Commun. Math. Phys. 94(1), 61-66 (1984)

4. Benjamin, T.B.: Impulse, flow force and variational principles. IMA J. Appl. Math. 32(1-3), 3-68 (1984)

5. Bona, J.L., Smith, R.: The initial-value problem for the Korteweg-de Vries equation. Philos. Trans. R. Soc. Lond. Ser. A 278(1287), 555-601 (1975)

6. Brenner, M., Hormoz, S., Pumir, A.: A potential singularity mechanism for the Euler Equations (preprint)

7. Caffarelli, L., Kohn, R., Nirenberg, L.: Partial regularity of suitable weak solutions of the Navier-Stokes equations. Commun. Pure Appl. Math. 35(6), 771-831 (1982)

8. Castro, A., Córdoba, D., Gancedo, F.: Singularity formations for a surface wave model. Nonlinearity 23(11), 2835-2847 (2010)

9. Chae, D.: On the continuation principles for the Euler equations and the quasi-geostrophic equation. J. Differ. Equ. 227, 640-651 (2006)

10. Chae, D.: Nonexistence of self-similar singularities for the 3D incompressible Euler equations. Commun. Math. Phys. 273, 203-215 (2007)

11. Chae, D.: Handbook of Differential Equations: Evolutionary Equations. Incompressible Euler Equations: The Blow-Up Problem and Related Results, vol. IV, p. 155. Elsevier, Amsterdam (2008)

12. Chae, D.: On the generalized self-similar singularities for the Euler and the Navier-Stokes equations. J. Funct. Anal. 258, 2865-2883 (2010)

13. Chae, D.: On the self-similar solutions of the 3D Euler and the related equations. Commun. Math. Phys. 305, 333-349 (2011)

14. Chae, D., Constantin, P., Córdoba, D., Gancedo, F., Wu, J.: Generalized surface quasi-geostrophic equations with singular velocities. Commun. Pure Appl. Math. 65(8), 1037-1066 (2012)

15. Chae, D., Constantin, P., Wu, J.: Inviscid models generalizing the two-dimensional Euler and the surface quasi-geostrophic equations. Arch. Ration. Mech. Anal. 202(1), 35-62 (2011)

16. Chae, D., Constantin, P., Wu, J.: Dissipative models generalizing the 2D Navier-Stokes and surface quasi-geostrophic equations. Indiana Univ. Math. J. 61(5), 1997-2018 (2012)

17. Choi, K., Kiselev, A., Yao, Y.: Finite time blowup for a 1D model of 2D Boussinesq system. Commun. Math. Phys. 334, 1667-1679 (2015)

18. Choi, K., Hou, T., Kiselev, A., Luo, G., Sverak, V., Yao, Y.: On the finite-time blowup of a 1D model for the 3D axisymmetric Euler equations (preprint)

19. Constantin, A., Kolev, B.: Geodesic flow on the diffeomorphism group of the circle. Comment Math. Helv. 78(4), 787-804 (2003)

20. Constantin, P.: On the Euler equations of incompressible fluids. Bull. Am. Math. Soc. (N.S.) 44(4), 603-621 (2007)

21. Constantin, P., Fefferman, C., Majda, A.: Geometric constrraints on potentially singular solutions for the 3-D Euler equation. Commun. PDE 21, 559-571 (1996)

22. Constantin, P., Iyer, G., Wu, J.: Global regularity for a modified critical dissipative quasi-geostrophic equation. Indiana Univ. Math. J. 57(6), 2681-2692 (2008)

23. Constantin, P., Lax, P.D., Majda, A.: A simple one-dimensional model for the three-dimensional vorticity equation. Commun. Pure Appl. Math. 38, 715-724 (1985)

24. Constantin, P., Majda, A., Tabak, E.: Formation of strong fronts in the 2-D quasigeostrophic thermal active scalar. Nonlinearity 7(6), 1495-1533 (1994)

25. Córdoba, D.: Nonexistence of simple hyperbolic blow-up for the quasi-geostrophic equation. Ann. Math. (2) 148(3), 1135-1152 (1998) 
26. Córdoba, D.: On the search for singularities in incompressible flows. Appl. Math. 51(4), 299-320 (2006)

27. Córdoba, A., Córdoba, D.: A maximum principle applied to quasi-geostrophic equations. Commun. Math. Phys. 249(3), 511-528 (2004)

28. Córdoba, A., Córdoba, D., Fontelos, M.: Formation of singularities for a transport equation with nonlocal velocity. Ann. Math. (2) 162(3), 1377-1389 (2005)

29. Córdoba, D., Fefferman, C.: On the collapse of tubes carried by 3D incompressible flows. Commun. Math. Phys. 222(2), 293-298 (2001)

30. Córdoba, D., Fontelos, M., Mancho, A., Rodrigo, J.: Evidence of singularities for a family of contour dynamics equations. Proc. Natl. Acad. Sci. USA 102(17), 5949-5952 (2005)

31. David, G., Journé, J.-L.: A boundedness criterion for generalized Calderón-Zygmund operators. Ann. Math. 120, 371-397 (1984)

32. De Gregorio, S.: On a one-dimensional model for the three-dimensional vorticity equation. J. Stat. Phys. 59, 1251-1263 (1990)

33. De Gregorio, S.: A partial differential equation arising in a $1 \mathrm{D}$ model for the $3 \mathrm{D}$ vorticity equation. Math. Method Appl. Sci. 19, 1233-1255 (1996)

34. Deng, J., Hou, T., Yu, X.: Geometric properties and nonblowup of 3D incompressible Euler flow. Commun. PDE 30, 225-243 (2005)

35. Dong, H., Du, D., Li, D.: Finite time singularities and global well-posedness for fractal Burgers equations. Indiana Univ. Math. J. 58, 807-821 (2009)

36. Dong, H., Li, D.: On a one-dimensional $\alpha$-patch model with nonlocal drift and fractional dissipation. Trans. Am. Math. Soc. 366(4), 2041-2061 (2014)

37. Ebin, D.G., Marsden, J.: Groups of diffeomorphisms and the motion of an incompressible fluid. Ann. Math. 2(92), 102-163 (1970)

38. Escher, J., Kolev, B., Wunsch, M.: The geometry of a vorticity model equation. Commun. Pure Appl. Anal. 11(4), 1407-1419 (2012)

39. Friedlander, S., Gancedo, F., Sun, W., Vicol, V.: On a singular incompressible porous media equation. J. Math. Phys. 53(11), 115602 (2012)

40. Friedlander, S., Rusin, W.: On the second iterate for critically diffusive active scalar equations. J. Math. Fluid Mech. 15(3), 481-492 (2013)

41. Gancedo, F.: Existence for the $\alpha$-patch model and the QG sharp front in Sobolev spaces. Adv. Math. 217(6), 2569-2598 (2008)

42. Gancedo, F., Strain, R.: Absence of splash singularities for SQG sharp fronts and the Muskat problem (preprint)

43. Hou, T.Y., Lei, Z.: On the stabilizing effect of convection in three-dimensional incompressible flows. Commun. Pure Appl. Math. 62(4), 501-564 (2009)

44. Hou, T., Liu, P.: Self-similar singularity of a 1D model for the 3D axisymmetric Euler equations. Res. Math. Sci. 2, 5 (2015)

45. Hou, T., Shi, Z., Wang, S.: On singularity formation of a 3D model for incompressible Navier-Stokes equations. Adv. Math. 230(2), 607-641 (2012)

46. Katz, N., Pavlovic, N.: Finite time blow-up for a dyadic model of the Euler equations. Trans. Am. Math. Soc. 357, 695-708 (2005)

47. Khesin, B., Wendt, R.: The Geometry of Infinite-Dimensional Groups. Ergebnisse der Mathematik und ihrer Grenzgebiete. 3. Folge. A Series of Modern Surveys in Mathematics, vol. 51. Springer, Berlin (2009)

48. Kiselev, A.: Regularity and blow up for active scalars. Math. Model. Nat. Phenom. 5(4), 225-255 (2010)

49. Kiselev, A., Nazarov, F.: A Simple Energy Pump for the Surface Quasi-geostrophic Equation. Nonlinear Partial Differential Equations. Abel Symposia, vol. 7, pp. 175-179. Springer, Heidelberg (2012)

50. Kiselev, A., Nazarov, F., Shterenberg, R.: Blow up and regularity for fractal Burgers equation. Dyn. Partial Differ. Equ. 5, 211-240 (2008)

51. Kiselev, A., Ryzhik, L., Yao, Y., Zlatos, A.: Finite time singularity for the modified SQG patch equation (preprint)

52. Klainerman, S., Majda, A.: Singular limits of quasilinear hyperbolic systems with large parameters and the incompressible limit of compressible fluids. Commun. Pure Appl. Math. 34, 481-524 (1981)

53. Kato, T., Ponce, G.: Commutator estimates and the Euler and Navier-Stokes equations. Commun. Pure Appl. Math. 41, 891-907 (1988) 
54. Lenells, J.: The Hunter-Saxton equation describes the geodesic flow on a sphere. J. Geom. Phys. 57(10), 2049-2064 (2007)

55. Li, D., Rodrigo, J.: Blow-up of solutions for a 1D transport equation with nonlocal velocity and supercritical dissipation. Adv. Math. 217, 2563-2568 (2008)

56. Luo, G., Hou, T.: Toward the finite-time blowup of the 3D axisymmetric Euler equations: a numerical investigation. Multiscale Model. Simul. 12, 1722-1776 (2014)

57. Luo, G., Hou, T.: Potentially singular solutions of the $3 \mathrm{~d}$ axisymmetric Euler equations. Proc. Natl. Acad. Sci. USA 111, 12968-12973 (2014)

58. Majda, A., Bertozzi, A.: Vorticity and Incompressible Flow. Cambridge Texts in Applied Mathematics, vol. 27. Cambridge University Press, Cambridge (2002)

59. Okamoto, H., Sakajo, T., Wunsch, M.: On a generalization of the Constantin-Lax-Majda equation. Nonlinearity 21(10), 2447-2461 (2008)

60. Preston, S., Sarria, A.: Lagrangian aspects of the axisymmetric Euler equation (preprint)

61. Shankar, R.: Symmetries and conservation laws of the Euler equations in Lagrangian coordinates (preprint)

62. Stein, E.: Harmonic Analysis: Real-Variable Methods, Orthogonality, and Oscillatory Integrals. With the Assistance of Timothy S. Murphy. Princeton Mathematical Series, 43 Monographs in Harmonic Analysis, III, Princeton University Press, Princeton (1993)

63. Sukatme, J., Smith, L.: Local and Nonlocal Dispersive Turbulence (preprint). arXiv:0709.2897

64. Tao, T.: Finite time blowup for an averaged three-dimensional Navier-Stokes equation. J. Am. Math. Soc. 29(3), 601-674 (2016)

65. Tao, T.: Noether's theorem and the conservation laws for the Euler equations, terrytao.wordpress.com/2014/03/02

66. Tao, T.: Finite time blowup for a supercritical defocusing nonlinear wave system (preprint)

67. Tao, T.: Finite time blowup for a high dimensional nonlinear wave systems with bounded smooth nonlinearity (preprint)

68. Taylor, M.: Tools for PDE: Pseudodifferential Operators, Paradifferential Operators, and Layer Potentials, Mathematical Surveys and Monographs, vol. 81. American Mathematical Society, Providence (2000)

69. Turkington, B.: Vortex rings with swirl: axisymmetric solutions of the Euler equations with nonzero helicity. SIAM J. Math. Anal. 20(1), 57-73 (1989)

70. Tzvetkov, N.: Ill-posedness issues for nonlinear dispersive equations. Lectures on nonlinear dispersive equations. GAKUTO Int. Ser. Math. Sci. Appl. 27, 63-103 (2006)

71. Washabaugh, P.: The SQG equation as a geodesic equation (preprint)

72. Wu, J.: Solutions of the 2D quasi-geostrophic equation in Hölder spaces. Nonlinear Anal. 62(4), 579594 (2005)

73. Wunsch, M.: On the geodesic flow on the group of diffeomorphisms of the circle with a fractional Sobolev right-invariant metric. J. Nonlinear Math. Phys. 17(1), 7-11 (2010)

74. Wunsch, M.: The generalized Constantin-Lax-Majda equation revisited. Commun. Math. Sci. 9, 929936 (2011) 